3 Model development

4 General

Ecosys is an hourly time-step model with multiple canopy and soil layers that

6 provide a framework for different plant and microbial populations to acquire, transform

7 and exchange resources (energy, water, $\mathrm{C}, \mathrm{N}$ and $\mathrm{P}$ ). The model is constructed from

8 algorithms representing basic physical, chemical and biological processes that determine

9 process rates in plant and microbial populations interacting within complex biomes. These

10 algorithms interact to simulate complex ecosystem behaviour across a wide range of

11 spatial and biological scales. The model is designed to represent terrestrial ecosystems

12 under range of natural and anthropogenic disturbances and environmental changes at patch

13 (spatially homogenous one-dimensional) and landscape (spatially variable two- or three-

14 dimensional) scales. A comprehensive description of ecosys with a detailed listing of

15 inputs, outputs, governing equations, parameters, results and references can be found in

16 Grant (2001). A more detailed description of model algorithms and parameters most

17 relevant to simulating temperature, water and nutrient effects on net ecosystem

18 productivity (NEP) is given below, with reference to equations and variable definitions in

19 appendices A-H below.

20 Appendix A: Soil $\mathbf{C}, \mathbf{N}$ and $\mathbf{P}$ transformations

\title{
21 Decomposition
}

Organic transformations in ecosys occur in five organic matter-microbe

23 complexes (coarse woody litter, fine non-woody litter, animal manure, particulate organic 
24 matter (POM), and humus) in each soil layer. Each complex consists of five organic

25 states: solid organic matter $(S)$, dissolved organic matter $(Q)$, sorbed organic matter $(A)$,

26 microbial biomass $(M)$, and microbial residues $(Z)$, among which $\mathrm{C}, \mathrm{N}$, and $\mathrm{P}$ are

27 transformed. Organic matter in litter and manure complexes are partitioned from

28 proximate analysis results into carbohydrate, protein, cellulose, and lignin components of

29 differing vulnerability to hydrolysis. Organic matter in POM, humus, microbial biomass

30 and microbial residues in all complexes are also partitioned into components of differing

31 vulnerability to hydrolysis.

32 The rate at which each component of each organic state in each complex is

33 hydrolyzed during decomposition is a first-order function of the decomposer biomass $(M)$

34 of all heterotrophic microbial populations [A1]. Decomposer biomasses are redistributed

35 among complexes from active biomasses according to biomass-substrate concentration

36 differences (priming) [A3]. The rate at which each component is hydrolyzed is also a

37 Monod function of substrate concentration [A3, A5], calculated from the fraction of

38 substrate mass colonized by $M$ [A4]. Hydrolysis rates are controlled by soil temperature

$39\left(T_{s}\right)$ through an Arrhenius function [A6] and by soil water content $(\theta)$ through its effect

40 on aqueous microbial concentrations ([M]) $[\mathrm{A} 3, \mathrm{~A} 5]$ in surface litter and in a spatially

41 resolved soil profile. $T_{s}$ and $\theta$ are calculated from surface energy balances and from heat

42 and water transfer schemes through canopy-snow-residue-soil profiles. Release of $\mathrm{N}$ and

$43 \mathrm{P}$ from hydrolysis of each component in each complex is determined by its $\mathrm{N}$ and $\mathrm{P}$

44 concentrations [A7] which are determined from those of the originating litterfall as

45 described in Autotrophic respiration and Growth and senescence below. Most non-lignin

46 hydrolysis products are released as dissolved organic C, N and P (DOC, DON, and DOP) 
47 which are adsorbed or desorbed according to a power function of their soluble 48 concentrations [A8-A10].

\section{Microbial growth}

The DOC decomposition product is the substrate for heterotrophic respiration $\left(R_{h}\right)$

51 by all $M$ in each substrate-microbe complex [A13]. Total $R_{h}$ for all soil layers [A11]

52 drives $\mathrm{CO}_{2}$ emission from the soil surface through volatilization and diffusion. $R_{h}$ may be

53 constrained by microbial $\mathrm{N}$ or $\mathrm{P}$ concentrations, $T_{s}$, DOC and $\mathrm{O}_{2}$ [A12 - A14]. $\mathrm{O}_{2}$ uptake

54 by $M$ is driven by $R_{h}$ [A16] and constrained by $\mathrm{O}_{2}$ diffusivity to microbial surfaces [A17],

55 as described for roots in Autotrophic respiration and Growth and senescence below.

56 Thus $R_{h}$ is coupled to $\mathrm{O}_{2}$ reduction by all aerobic $M$ according to $\mathrm{O}_{2}$ availability. $R_{h}$ not

57 coupled with $\mathrm{O}_{2}$ reduction is coupled with the sequential reduction of $\mathrm{NO}_{3}{ }^{-}, \mathrm{NO}_{2}^{-}$, and

$58 \quad \mathrm{~N}_{2} \mathrm{O}$ by heterotrophic denitrifiers, and with the reduction of organic $\mathrm{C}$ by fermenters and

59 acetotrophic methanogens. In addition, autotrophic nitrifiers conduct $\mathrm{NH}_{4}{ }^{+}$and $\mathrm{NO}_{2}{ }^{-}$

60 oxidation, and $\mathrm{NO}_{2}{ }^{-}$reduction, and autotrophic methanogens and methanotrophs conduct

$61 \mathrm{CH}_{4}$ production and oxidation.

All microbial populations undergo maintenance respiration $\left(R_{m}\right)$ [A18, A19],

63 depending on microbial $\mathrm{N}$ and $T_{\mathrm{s}}$ as described earlier for plants. $R_{h}$ in excess of $R_{m}$ is

64 used in growth respiration $R_{\mathrm{g}}$ [A20], the energy yield $\Delta G$ of which drives growth in

65 biomass $M$ from DOC uptake according to the energy requirements of biosynthesis [A21-

66 A22]. $R_{m}$ in excess of $R_{h}$ causes microbial dieback. $M$ also undergoes first-order

67 decomposition $\left(D_{m}\right)$ [A23]. Internal retention and recycling of microbial $\mathrm{N}$ and $\mathrm{P}$ during

68 decomposition [A24] is modelled whenever these nutrients constrain $R_{h}$ [A12]. Changes 
69 in $M$ arise from differences between gains from DOC uptake and losses from $R_{m}+R_{\mathrm{g}}+$ $70 D_{m}[\mathrm{~A} 25]$

\section{Microbial nutrient exchange}

72 During these changes, all microbial populations seek to maintain set minimum 73 ratios of $\mathrm{C}: \mathrm{N}$ or $\mathrm{C}: \mathrm{P}$ in $M$ by mineralizing or immobilizing $\mathrm{NH}_{4}^{+}, \mathrm{NO}_{3}^{-}$and $\mathrm{H}_{2} \mathrm{PO}_{4}^{-}$

74 [A26], thereby controlling solution $\left[\mathrm{NH}_{4}^{+}\right],\left[\mathrm{NO}_{3}{ }^{-}\right]$and $\left[\mathrm{H}_{2} \mathrm{PO}_{4}{ }^{-}\right]$that determine root and 75 mycorrhizal uptake. If immobilization is inadequate to maintain these minimum ratios, 76 then biomass C:N or C:P may rise, but $R_{h}$ is constrained by $\mathrm{N}$ or $\mathrm{P}$ present in the lowest 77 concentration with respect to that at the minimum ratio [A12]. Non-symbiotic 78 heterotrophic diazotrophs can also fix aqueous $\mathrm{N}_{2}$ [A27] to the extent that immobilization 79 is inadequate to maintain their set minimum $\mathrm{C}: \mathrm{N}$, but at an additional respiration cost 80 [A28]. Changes in microbial $\mathrm{N}$ and $\mathrm{P}$ arise from DON and DOP uptake plus $\mathrm{NH}_{4}{ }^{+}, \mathrm{NO}_{3}{ }^{-}$ 81 and $\mathrm{H}_{2} \mathrm{PO}_{4}{ }^{-}$immobilization and $\mathrm{N}_{2}$ fixation, less $\mathrm{NH}_{4}{ }^{+}, \mathrm{NO}_{3}{ }^{-}$, and $\mathrm{H}_{2} \mathrm{PO}_{4}{ }^{-}$mineralization 82 and microbial $\mathrm{N}$ and $\mathrm{P}$ decomposition [A29].

\section{Humification}

$\mathrm{C}, \mathrm{N}$ and $\mathrm{P}$ decomposition products in each organic matter-microbe complex are

85 gradually stabilized into more recalcitrant organic forms with lower C:N and C:P ratios.

86 Products from lignin hydrolysis [A1, A7] combine with some of the products from 87 protein and carbohydrate hydrolysis in the litterfall and manure complexes and are 88 transferred to the POM complex [A31-A34]. Microbial decomposition products [A2389 A24] from all complexes are partitioned between the humus complex and microbial 90 residues in the originating complex according to soil clay content [A35-A36].

\section{Appendix B: Soil-plant water relations}




\section{Canopy transpiration}

93 Canopy energy exchange in ecosys is calculated from an hourly two-stage

94 convergence solution for the transfer of water and heat through a multi-layered multi-

95 population soil-root-canopy system. The first stage of this solution requires convergence

96 to a value of canopy temperature $\left(T_{\mathrm{c}}\right)$ for each plant population at which the first-order

97 closure of the canopy energy balance (net radiation $\left(R_{n}\right)$, latent heat flux $(L E)$ [B 1a,b,c],

98 sensible heat flux $(H)$ [B1d], and change in heat storage $(G))$ is achieved. These fluxes

99 are controlled by aerodynamic $\left(r_{\mathrm{a}}\right)$ [B3] and canopy stomatal $\left(r_{\mathrm{c}}\right)$ [B2] resistances. Two

100 controlling mechanisms are postulated for $r_{\mathrm{c}}$ which are solved in two successive steps:

101 (1) At the leaf level, leaf resistance $\left(r_{1}\right)$ [C4] controls gaseous $\mathrm{CO}_{2}$ diffusion through each 102 leaf surface when calculating $\mathrm{CO}_{2}$ fixation [C1] from concurrent solutions for 103 diffusion $\left(V_{\mathrm{g}}\right)[\mathrm{C} 2]$ and carboxylation $\left(V_{\mathrm{c}}\right)$ [C3]. The value of $r_{1}$ is calculated from a 104 minimum leaf resistance $\left(r_{\text {lmin }}\right)$ [C5] for each leaf surface that allows a set ratio for 105 intercellular to canopy $\mathrm{CO}_{2}$ concentration $\left(C_{\mathrm{i}}^{\prime}: C_{\mathrm{b}}\right)$ to be maintained at $V_{\mathrm{c}}$ under 106 ambient irradiance, air temperature $T_{a}, C_{a}$ and zero canopy water potential $\left(\psi_{\mathrm{c}}\right)\left(V_{\mathrm{c}}^{\prime}\right)$. 107 This ratio will be allowed to vary diurnally as described in $C_{3}$ gross primary 108 productivity when $\psi_{\mathrm{c}}$ is solved in the second stage of the convergence solution. 109 Values of $r_{\text {lmin }}$ are aggregated by leaf surface area to a canopy value $\left(r_{\mathrm{cmin}}\right)$ for use in 110 the energy balance convergence scheme [B2a].

111 (2) At the canopy level, $r_{\mathrm{c}}$ rises from $r_{\mathrm{cmin}}$ at zero $\psi_{\mathrm{c}}$ from step (1) above through an 112 exponential function of canopy turgor potential $\left(\psi_{\mathrm{t}}\right)[\mathrm{B} 2 \mathrm{~b}]$ calculated from $\psi_{\mathrm{c}}$ and 113 osmotic water potential $(\psi \pi)$ [B4] during convergence for transpiration vs. water $114 \quad$ uptake. 


\section{Root and mycorrhizal water uptake}

116 Root and mycorrhizal water uptake $(U)$ [B5] is calculated from the difference

117 between $\psi_{\mathrm{c}}$ and soil water potential $\left(\psi_{\mathrm{s}}\right)$ across soil and root hydraulic resistances $\left(\Omega_{\mathrm{s}}\right.$

118 [B9] and $\left.\Omega_{\mathrm{r}}[\mathrm{B} 10-\mathrm{B} 12]\right)$ in each rooted soil layer [B6]. Root resistances are calculated 119 from root radial [B10] and from primary [B11] secondary [B12] axial resistivities using

120 root lengths and surface areas from a root system submodel [B13] driven by exchange of

121 nonstructural $\mathrm{C}, \mathrm{N}$ and $\mathrm{P}$ along concentration gradients generated by uptake vs.

122 consumption of $\mathrm{C}, \mathrm{N}$ and $\mathrm{P}$ in shoots and roots (Grant, 1998a).

123 Canopy water potential

124 After convergence for $T_{\mathrm{c}}$ is achieved, the difference between canopy transpiration

$125\left(E_{\mathrm{c}}\right)$ from the energy balance [B1] and total root water uptake $\left(U_{\mathrm{c}}\right)$ [B5] from all rooted 126 layers in the soil is tested against the difference between canopy water content from the 127 previous hour and that from the current hour [B14]. This difference is minimized in each 128 iteration by adjusting $\psi_{\mathrm{c}}$ which in turn determines each of the three terms in [B14].

129 Because $r_{\mathrm{c}}$ and $T_{\mathrm{c}}$ both drive $E_{\mathrm{c}}$, the canopy energy balance described in Canopy 130 transpiration is recalculated for each adjusted value of $\psi_{\mathrm{c}}$ during convergence.

131 Appendix C: Gross primary productivity and autotrophic respiration

$132 \mathbf{C}_{\mathbf{3}}$ gross primary productivity

133 After successful convergence for $T_{\mathrm{c}}$ and $\psi_{\mathrm{c}}$ as described in Canopy transpiration, $134 V_{\mathrm{c}}$ is recalculated from that under zero $\psi_{\mathrm{c}}\left(V_{\mathrm{c}}^{\prime}\right)$ to that under ambient $\psi_{\mathrm{c}}$. This 135 recalculation is driven by stomatal effects on $V_{\mathrm{g}}[\mathrm{C} 2]$ from the increase in $r_{\mathrm{Imin}}$ at zero $\psi_{\mathrm{c}}$ 136 [C5] to $r_{\mathrm{c}}$ at ambient $\psi_{\mathrm{c}}$ [C4], and by non-stomatal effects $\left(f_{\psi}\right)$ [C9] on $\mathrm{CO}_{2^{-}}$and light137 limited carboxylation ( $\left.V_{\mathrm{b}}\right)$ [C6] and $V_{\mathrm{j}}$ [C7] (Grant and Flanagan, 2007). The 
138 recalculation of $V_{\mathrm{c}}$ is accomplished through a convergence solution for $C_{\mathrm{i}}$ and its aqueous

139 counterpart $C_{\mathrm{c}}$ at which $V_{\mathrm{g}}$ [C2] equals $V_{\mathrm{c}}$ [C3] (Grant and Flanagan, 2007). The $\mathrm{CO}_{2}$

140 fixation rate of each leaf surface at convergence is added to arrive at a value for gross

141 primary productivity (GPP) by each plant population in the model [C1]. The $\mathrm{CO}_{2}$ fixation

142 product is stored in nonstructural $\mathrm{C}$ pools $\left(\sigma_{\mathrm{C}}\right)$ in each branch.

143 GPP is strongly controlled by nutrient uptake $\left(U_{\mathrm{NH}_{4}}, U_{\mathrm{NO}_{3}}\right.$ and $\left.U_{\mathrm{PO}_{4}}[\mathrm{C} 23]\right)$,

144 products of which are added to nonstructural $\mathrm{N}\left(\sigma_{\mathrm{N}}\right)$ and $\mathrm{P}\left(\sigma_{\mathrm{P}}\right)$ in root and mycorrhizal

145 layers where they are coupled with $\sigma_{\mathrm{C}}$ to drive growth of branches, roots and 146 mycorrhizae as described in Growth and senescence. Low $\sigma_{\mathrm{N}}: \sigma_{\mathrm{C}}$ or $\sigma_{\mathrm{P}}: \sigma_{\mathrm{C}}$ in branches 147 indicate excess $\mathrm{CO}_{2}$ fixation with respect to $\mathrm{N}$ or $\mathrm{P}$ uptake for phytomass growth. Such 148 ratios in the model have two effects on GPP:

149 (1) They reduce activities of rubisco [C6a] and chlorophyll [C7a] through product 150 inhibition [C11], thereby simulating the suppression of $\mathrm{CO}_{2}$ fixation by leaf $\sigma_{\mathrm{C}}$ 151 accumulation widely reported in the literature.

152 (2) They reduce the structural N:C and P:C ratios at which leaves are formed because $\sigma_{\mathrm{C}}$, $153 \sigma_{\mathrm{N}}$ and $\sigma_{\mathrm{P}}$ are the substrates for leaf growth. Lower structural ratios cause a 154 proportional reduction in areal concentrations of rubisco [C6b] and chlorophyll [C7b], 155 reducing leaf $\mathrm{CO}_{2}$ fixation.

\section{Autotrophic respiration}

157 The temperature dependent oxidation of these nonstructural pools $\left(R_{\mathrm{c}}\right)$ [C14], plus 158 the energy costs of nutrient uptake [C23], drive autotrophic respiration $\left(R_{a}\right)$ [C13] by all 159 branches, roots and mycorrhizae. $R_{\mathrm{c}}$ by roots and mycorrhizae is constrained by $\mathrm{O}_{2}$ 160 uptake $\left(U_{\mathrm{O} 2}\right)[\mathrm{C} 14 \mathrm{~b}]$ calculated by solving for aqueous $\mathrm{O}_{2}$ concentrations at root and 
161 mycorrhizal surfaces $\left(\left[\mathrm{O}_{2 \mathrm{r}}\right]\right)$ at which convection + radial diffusion through the soil 162 aqueous phase plus radial diffusion through the root aqueous phase [C14d] equals active 163 uptake driven by $\mathrm{O}_{2}$ demand from $R_{\mathrm{c}}[\mathrm{C} 14 \mathrm{c}]$ (Grant, 2004). These diffusive fluxes are in 164 turn coupled to volatilization -dissolution between aqueous and gaseous phases in soil 165 and root [D14]. The diffusion processes are driven by aqueous $\mathrm{O}_{2}$ concentrations 166 sustained by transport and dissolution of gaseous $\mathrm{O}_{2}$ through soil and roots (Grant, 2004), 167 and are governed by lengths and surface areas of roots and mycorrhizae (Grant, 1998a).

168 Thus $R_{\mathrm{c}}$ is coupled to $\mathrm{O}_{2}$ reduction by all root and mycorrhizal populations according to $169 \mathrm{O}_{2}$ availability. $R_{\mathrm{c}}$ is first used to meet maintenance respiration requirements $\left(R_{\mathrm{m}}\right)$, 170 calculated independently of $R_{\mathrm{c}}$ from the $\mathrm{N}$ content in each organ, and a function of $T_{c}$ or

$171 T_{s}$ [C16]. Any excess of $R_{\mathrm{c}}$ over $R_{\mathrm{m}}$ is expended as growth respiration $\left(R_{\mathrm{g}}\right)$, constrained 172 by branch, root or mycorrhizal $\psi_{t}$ [C17]. When $R_{\mathrm{m}}$ exceeds $R_{\mathrm{c}}$, the shortfall is met by the 173 respiration of remobilizable $\mathrm{C}\left(R_{\mathrm{S}}\right)$ in leaves and twigs or roots and mycorrhizae [C15].

\section{Growth and senescence}

$175 R_{\mathrm{g}}$ drives the conversion of branch $\sigma_{\mathrm{C}}$ into foliage, twigs, branches, boles and 176 reproductive material according to organ growth yields $\left(Y_{\mathrm{g}}\right)$ and phenology-dependent 177 partitioning coefficients [C20], and the conversion of root and mycorrhizal $\sigma_{\mathrm{C}}$ into 178 primary and secondary axes according to root and mycorrhizal growth yields. Growth 179 also requires organ-specific ratios of nonstructural $\mathrm{N}\left(\sigma_{\mathrm{N}}\right)$ and $\mathrm{P}\left(\sigma_{\mathrm{P}}\right)$ from $U_{\mathrm{NH}_{4}}, U_{\mathrm{NO}_{3}}$ 180 and $U_{\mathrm{PO}_{4}}$ [C23] which are coupled with $\sigma_{\mathrm{C}}$ to drive growth of branches, roots and 181 mycorrhizae.

182 The translocation of $\sigma_{\mathrm{C}}, \sigma_{\mathrm{N}}$ and $\sigma_{\mathrm{P}}$ among branches and root and mycorrhizal 183 layers is driven by concentration gradients generated by production of $\sigma_{\mathrm{C}}$ from branch 
184 GPP and of $\sigma_{\mathrm{N}}$ and $\sigma_{\mathrm{P}}$ from root and mycorrhizal uptake vs. consumption of $\sigma_{\mathrm{C}}, \sigma_{\mathrm{N}}$ and

$185 \sigma_{\mathrm{P}}$ from $R_{c}, R_{g}$ and phytomass growth (Grant, 1998a). Low $\sigma_{\mathrm{N}}: \sigma_{\mathrm{C}}$ or $\sigma_{\mathrm{P}}: \sigma_{\mathrm{C}}$ in 186 mycorrhizae and roots indicates inadequate $\mathrm{N}$ or $\mathrm{P}$ uptake with respect to $\mathrm{CO}_{2}$ fixation. 187 These ratios affect translocation of $\sigma_{\mathrm{C}}, \sigma_{\mathrm{N}}$ and $\sigma_{\mathrm{P}}$ by lowering mycorrhizal-root-branch 188 concentration gradients of $\sigma_{\mathrm{N}}$ and $\sigma_{\mathrm{P}}$ while raising branch-root-mycorrhizal concentration 189 gradients of $\sigma_{\mathrm{C}}$. These changes slow transfer of $\sigma_{\mathrm{N}}$ and $\sigma_{\mathrm{P}}$ from root to branch and hasten 190 transfer of $\sigma_{\mathrm{C}}$ from branch to root, increasing root and mycorrhizal growth at the expense 191 of branch growth, and thereby raising $\mathrm{N}$ and $\mathrm{P}$ uptake [C23] with respect to $\mathrm{CO}_{2}$ fixation.

192 Conversely, high $\sigma_{\mathrm{N}}: \sigma_{\mathrm{C}}$ or $\sigma_{\mathrm{P}}: \sigma_{\mathrm{C}}$ in roots and mycorrhizae indicate excess $\mathrm{N}$ or $\mathrm{P}$ uptake 193 with respect to $\mathrm{CO}_{2}$ fixation. Such ratios reduce specific activities of root and 194 mycorrhizal surfaces for $\mathrm{N}$ or $\mathrm{P}$ uptake through a product inhibition function as has been 195 observed experimentally. These changes hasten transfer of $\sigma_{\mathrm{N}}$ and $\sigma_{\mathrm{P}}$ from root to branch 196 and slow transfer of $\sigma_{\mathrm{C}}$ from branch to root, increasing branch growth at the expense of 197 root and mycorrhizal growth, and thereby slowing $\mathrm{N}$ and $\mathrm{P}$ uptake Thus the modelled 198 plant translocates $\sigma_{\mathrm{C}}, \sigma_{\mathrm{N}}$ and $\sigma_{\mathrm{P}}$ among branches, roots and mycorrhizae to maintain a 199 functional equilibrium between acquisition and use of $\mathrm{C}, \mathrm{N}$ and $\mathrm{P}$ by different parts of the 200 plant.

$201 \quad R_{\mathrm{g}}$ is limited by $\psi_{t}[\mathrm{C} 17]$, and because branch $\psi_{t}$ declines relatively more with soil 202 drying than does root $\psi_{t}$, branch $R_{\mathrm{g}}$ also declines relatively more with soil drying than 203 does root $R_{\mathrm{g}}$, slowing oxidation of $\sigma_{\mathrm{C}}$ in branches and allowing more translocation of $\sigma_{\mathrm{C}}$ 204 from branches to roots. This change in allocation of $\sigma_{\mathrm{C}}$ enables more root growth to 205 reduce $\Omega_{\mathrm{s}}, \Omega_{\mathrm{r}}$ and $\Omega_{\mathrm{a}}$, and hence increase $U$ [B6], thereby offsetting the effects of soil 
drying on $\psi_{t}$. Thus the modelled plant translocates $\sigma_{\mathrm{C}}, \sigma_{\mathrm{N}}$ and $\sigma_{\mathrm{P}}$ among branches, roots

207 and mycorrhizae to maintain a functional equilibrium between acquisition and use of 208 water.

$209 R_{\mathrm{s}}[\mathrm{C} 15]$ drives the withdrawal of remobilizable C, N and P (mostly nonstructural 210 protein) from leaves and twigs or roots and mycorrhizae into $\sigma_{\mathrm{N}}$ and $\sigma_{\mathrm{P}}$, and the loss of 211 associated non-remobilizable $\mathrm{C}, \mathrm{N}$ and $\mathrm{P}$ (mostly structural) as litterfall [C18]. Provision 212 is also made to withdraw remobilizable $\mathrm{N}$ or $\mathrm{P}$ from leaves and twigs or roots and 213 mycorrhizae when ratios of $\sigma_{\mathrm{N}}: \sigma_{\mathrm{C}}$ or $\sigma_{\mathrm{P}}: \sigma_{\mathrm{C}}$ become smaller than those required for 214 growth of new phytomass. This withdrawal drives the withdrawal of associated 215 remobilizable $\mathrm{C}$, and the loss of associated non-remobilizable $\mathrm{C}, \mathrm{N}$ and $\mathrm{P}$ as litterfall. 216 Environmental constraints such as water, heat, nutrient or $\mathrm{O}_{2}$ stress that reduce $\sigma_{\mathrm{C}}$ and 217 hence $R_{\mathrm{c}}$ with respect to $R_{\mathrm{m}}$ therefore hasten litterfall.

$218 R_{a}$ of each branch or root and mycorrhizal layer is the total of $R_{c}$ and $R_{s}$, and net 219 primary productivity (NPP) is the difference between canopy GPP [C1] and total $R_{a}$ of all 220 branches and root and mycorrhizal layers [C13]. Phytomass net growth is the difference 221 between gains driven by $R_{\mathrm{g}}$ and $Y_{\mathrm{g}}$, and losses driven by $R_{\mathrm{s}}$ and litterfall [C20]. These 222 gains are allocated to leaves, twigs, wood and reproductive material at successive branch 223 nodes, and to roots and mycorrhizae at successive primary and secondary axes, driving 224 leaf expansion [C21a] and root extension [C21b]. Losses from remobilization and 225 litterfall in shoots start at the lowest node of each branch at which leaves or twigs are 226 present, and proceed upwards when leaves or twigs are lost. Losses in roots and 227 mycorrhizae start with secondary axes and proceeds to primary axes when secondary 228 axes are lost. 


\section{Root and mycorrhizal nutrient uptake}

230 Root and mycorrhizal uptake of $\mathrm{N}$ and $\mathrm{P}\left(U_{\mathrm{NH}_{4}}, U_{\mathrm{NO}_{3}}\right.$ and $\left.U_{\mathrm{PO}_{4}}\right)$ is calculated by

231 solving for solution $\left[\mathrm{NH}_{4}^{+}\right],\left[\mathrm{NO}_{3}{ }^{-}\right]$and $\left[\mathrm{H}_{2} \mathrm{PO}_{4}^{-}\right]$at root and mycorrhizal surfaces at

232 which radial transport by mass flow and diffusion from the soil solution to these surfaces

$233[\mathrm{C} 23 \mathrm{a}, \mathrm{c}, \mathrm{e}]$ equals active uptake by the surfaces [C23b,d,f]. Path lengths and surface areas

234 for $U_{\mathrm{NH}_{4}}, U_{\mathrm{NO}_{3}}$ and $U_{\mathrm{PO}_{4}}$ are calculated from a root and mycorrhizal growth submodel

235 driven by exchange of nonstructural $\mathrm{C}, \mathrm{N}$ and $\mathrm{P}$ along concentration gradients generated

236 by uptake vs. consumption of $\mathrm{C}, \mathrm{N}$ and $\mathrm{P}$ in shoots and roots (Grant, 1998a). A product

237 inhibition function is included to avoid uptake in excess of nutrient requirements [C23g].

$238 \mathbf{C}_{4}$ gross primary productivity

$239 \mathbf{C}_{4}$ mesophyll

240 In $\mathrm{C}_{4}$ plants, the mesophyll carboxylation rate is the lesser of $\mathrm{CO}_{2^{-}}$and light241 limited reaction rates [C26] (Berry and Farquhar, 1978). The $\mathrm{CO}_{2}$-limited rate is a 242 Michaelis-Menten function of PEP carboxylase (PEPc) activity and aqueous $\mathrm{CO}_{2}$ 243 concentration in the mesophyll [C29] parameterized from Berry and Farquhar (1978) and 244 from Edwards and Walker (1983). The light-limited rate [C30] is a hyperbolic function of 245 absorbed irradiance and mesophyll chlorophyll activity [C31] with a quantum 246 requirement based on 2 ATP from Berry and Farquhar (1978). PEPc [C32] and 247 chlorophyll [C33] activities are calculated from specific activities multiplied by set 248 fractions of leaf surface $\mathrm{N}$ density, and from functions of $\mathrm{C}_{4}$ product inhibition (Jiao and 249 Chollet, 1988; Lawlor, 1993) [C34], $\psi_{\mathrm{c}}([\mathrm{C} 35]$ as described in Grant and Flanagan 250 (2007)) and $T_{\mathrm{c}}[\mathrm{C} 36]$. Leaf surface $\mathrm{N}$ density is controlled by leaf structural N:C and P:C 
251 ratios calculated during leaf growth from leaf non-structural $\mathrm{N}: \mathrm{C}$ and $\mathrm{P}: \mathrm{C}$ ratios arising

252 from root $\mathrm{N}$ and $\mathrm{P}$ uptake (Grant, 1998a) vs. $\mathrm{CO}_{2}$ fixation.

\section{$253 \quad C_{4}$ mesophyll-bundle sheath exchange}

254 Differences in the mesophyll and bundle sheath concentrations of the $\mathrm{C}_{4}$ 255 carboxylation product drive mesophyll-bundle sheath transfer (Leegood, 2000) [C37]. 256 The bundle sheath concentration of the $\mathrm{C}_{4}$ product drives a product-inhibited 257 decarboxylation reaction (Laisk and Edwards, 2000) [C38], the $\mathrm{CO}_{2}$ product of which 258 generates a concentration gradient that drives leakage of $\mathrm{CO}_{2}$ from the bundle sheath to 259 the mesophyll [C39]. $\mathrm{CO}_{2}$ in the bundle sheath is maintained in 1:50 equilibrium with $260 \mathrm{HCO}_{3}^{-}$(Laisk and Edwards, 2000). At this stage of model development, the return of a $\mathrm{C}_{3}$ 261 decarboxylation product from the bundle sheath to the mesophyll is not simulated. 262 Parameters used in Eqs. [C37-C39] allowed mesophyll and bundle sheath concentrations 263 of $\mathrm{C}_{4}$ carboxylation products from [C40-C41] to be maintained at values consistent with 264 those in Leegood (2000), bundle sheath concentrations of $\mathrm{CO}_{2}$ (from [C42]) to be 265 maintained at values similar to those reported by Furbank and Hatch (1987), and bundle 266 sheath $\mathrm{CO}_{2}$ leakiness [C39]), expressed as a fraction of PEP carboxylation, to be

267 maintained at values similar to those in Williams et al. (2001), in sorghum as described in 268 Grant et al. (2004).

\section{$269 \mathbf{C}_{4}$ bundle sheath}

$270 \quad \mathrm{~A} \mathrm{C}_{3}$ model in which carboxylation is the lesser of $\mathrm{CO}_{2^{-}}$and light-limited reaction 271 rates (Farquhar et al., 1980) has been parameterized for the bundle sheath of $\mathrm{C}_{4}$ plants 272 [C43] from Seeman et al. (1984). The $\mathrm{CO}_{2}$-limited rate [C44] is a Michaelis-Menten 273 function of RuBP carboxylase (RuBPc) activity and bundle sheath $\mathrm{CO}_{2}$ concentration 
274 [C42]. The light-limited rate [C45] is a hyperbolic function of absorbed irradiance and 275 activity of chlorophyll associated with the bundle sheath with a quantum yield based on 3 276 ATP [C46]. The provision of reductant from the mesophyll to the bundle sheath in

277 NADP-ME species is not explicitly simulated. RuBPc [C47] and chlorophyll [C48] 278 activities are the products of specific activities and concentrations multiplied by set 279 fractions of leaf surface $\mathrm{N}$ density, and from functions of $\mathrm{C}_{3}$ product inhibition (Bowes, 280 1991; Stitt, 1991] [C49], $\psi_{\mathrm{c}}$ ( [A12] from Grant and Flanagan (2007)) and $T_{\mathrm{c}}$ [C36].

281 Rates of $\mathrm{C}_{3}$ product removal are controlled by phytomass biosynthesis rates 282 driven by concentrations of nonstructural products from leaf $\mathrm{CO}_{2}$ fixation and from root $283 \mathrm{~N}$ and $\mathrm{P}$ uptake. If biosynthesis rates are limited by nutrient uptake, consequent depletion 284 of nonstructural $\mathrm{N}$ or $\mathrm{P}$ and accumulation of nonstructural $\mathrm{C}$ will constrain specific 285 activities of RuBP and chlorophyll [C47-C49], and thereby slow $\mathrm{C}_{3}$ carboxylation [C43], 286 raise bundle sheath $\mathrm{CO}_{2}$ concentration [C42], accelerate $\mathrm{CO}_{2}$ leakage [C39], slow $\mathrm{C}_{4}$ 287 decarboxylation [C38], raise $\mathrm{C}_{4}$ product concentration in the bundle sheath [C41], slow $288 \mathrm{C}_{4}$ product transfer from the mesophyll [C37], raise $\mathrm{C}_{4}$ product concentration in the 289 mesophyll [C40], and slow mesophyll $\mathrm{CO}_{2}$ fixation [C32-C35]. This reaction sequence 290 simulates the progressive inhibition of $\mathrm{C}_{3}$ and $\mathrm{C}_{4}$ carboxylation hypothesized by Sawada 291 et al. (2002) following partial removal of $\mathrm{C}$ sinks in $\mathrm{C}_{4}$ plants.

292 Appendix D: Soil water, heat, gas and solute fluxes

\section{Surface water flux}

294 Surface runoff is modelled using Manning's equation [D1] with surface water 295 velocity (v) [D3] calculated from surface geometry [D5a] and slope [D5b], and with 
296 surface water depth $(d)$ [D2] calculated from surface water balance [D4] using kinematic

297 wave theory.

298 Subsurface water flux

299 Subsurface water flow [D7] is calculated from Richard's equation using bulk soil 300 water potentials $\left(\psi_{\mathrm{s}}\right)$ of both cells if both source and destination cells are unsaturated 301 [D9a], or Green-Ampt equation using $\psi_{\mathrm{s}}$ beyond the wetting front of the unsaturated cell 302 if either source or destination cell is saturated [D9b] (Grant et al., 2004). Subsurface 303 water flow can also occur through macropores using Poiseulle-Hagen theory for laminar 304 flow in tubes (Dimitrov et al., 2010), depending on inputs for macropore volume fraction.

\section{Exchange with water table}

306 If a water table is present in the model, subsurface boundary water fluxes between 307 saturated boundary grid cells and a fixed external water table are calculated from lateral 308 hydraulic conductivities of the grid cells, and from elevation differences and lateral 309 distances between the grid cells and the external water table [D10]. These terms are 310 determined from set values for the external water table depth $\left(\mathrm{WTD}_{\mathrm{x}}\right)$ of, and lateral 311 distance $\left(L_{\mathrm{t}}\right)$ to, an external water table.

\section{Surface heat flux}

313 Surface heat fluxes $(G)$ arising from closure of the energy balance at snowpack, 314 surface litter and soil surfaces [D11] (Grant et al., 1999) drive conductive-convective 315 fluxes among snowpack, surface litter and soil layers [D12]. These fluxes drive freezing316 thawing $\left(Q_{f}\right)$ and changes temperatures $(T)$ in snowpack, surface litter and soil layers 317 [D13].

\section{Gas flux}


All gases undergo volatilization-dissolution between the gaseous and aqueous

320 phases in the soil [D14a] and root [D14b], and between the atmosphere and the aqueous

321 phase at the soil surface [D15a], driven by gaseous-aqueous concentration differences

322 calculated from solubility coefficients and coupled to diffusive uptake by roots [C14] and

323 microbes [A17]. Gases also undergo convective-conductive transfer among soil layers

324 driven by gaseous concentration gradients and diffusivities [D16a,b,c] calculated from

325 air-filled porosities [D17a,b,c], and from each rooted soil layer directly to the atmosphere

326 through roots driven by gaseous concentration gradients and diffusivities [D16d]

327 calculated from root porosities [D17d]. Gases may also bubble upwards from soil zones

328 in which the total partial pressure of all aqueous gases exceeds atmospheric pressure

329 [D18].

330 Solute flux

332 among soil layers driven by aqueous concentration gradients and dispersivities [D19]

333 calculated from water-filled porosity [D20] and water flow length [D21].

334 Appendix E: Solute transformations

335 Precipitation-dissolution equilibria

336 Solution $\left[\mathrm{NH}_{4}^{+}\right],\left[\mathrm{NO}_{3}{ }^{-}\right]$and $\left[\mathrm{H}_{2} \mathrm{PO}_{4}{ }^{-}\right]$that drive $U_{\mathrm{NH}_{4}}, U_{\mathrm{NO}_{3}}$ and $U_{\mathrm{PO}_{4}}[\mathrm{C} 23]$ are

337 controlled by precipitation, adsorption and ion pairing reactions (Grant and Heaney,

338 1997; Grant et al., 2004), including precipitation-dissolution of $\mathrm{Al}(\mathrm{OH})_{3}, \mathrm{Fe}(\mathrm{OH})_{3}$,

$339 \mathrm{CaCO}_{3}, \mathrm{CaSO}_{4}, \mathrm{AlPO}_{4}, \mathrm{FePO}_{4}, \mathrm{Ca}\left(\mathrm{H}_{2} \mathrm{PO}_{4}\right)_{2}, \mathrm{CaHPO}_{4}$, and $\mathrm{Ca}_{5}\left(\mathrm{PO}_{4}\right)_{3} \mathrm{OH}$ [E1 - E9],

340 cation exchange between $\mathrm{Ca}^{2+}, \mathrm{NH}_{4}{ }^{+}$and other cations [E10 - E15], anion exchange 
341 between adsorbed and soluble $\mathrm{H}_{2} \mathrm{PO}_{4}{ }^{-}, \mathrm{HPO}_{4}{ }^{2-}$ and $\mathrm{OH}^{-}$[E16 - E20], and ion pairing $342[\mathrm{E} 22-\mathrm{E} 55]$.

343 Key governing equations for simulating net ecosystem productivity in ecosys.

344 Variables input to the model appear in bold with values given in the Definition of

$345 \quad$ Variables below.

346 Appendix F: $\mathbf{N}_{2}$ fixation

347 Rhizobial growth

348 Modelling the activity of symbiotic $\mathrm{N}_{2}$ fixing bacteria in roots follows a protocol 349 similar to that of non-symbiotic $\mathrm{N}_{2}$ fixing bacteria in soil. Respiration demand is driven 350 by specific activity, microbial biomass $\left(M_{\mathrm{n}}\right)$, and nonstructural $\mathrm{C}$ concentration $\left(\left[\chi_{\mathrm{n}}\right]\right)$ in 351 root nodules [F1], and is constrained by temperature [F2] and microbial $\mathrm{N}$ or $\mathrm{P}$ status 352 [F3]. Nodule respiration $(R)$ is constrained by the extent to which $\mathrm{O}_{2}$ uptake meets $\mathrm{O}_{2}$ 353 demand [F4] imposed by respiration demand [F5]. $\mathrm{O}_{2}$ uptake is in turn constrained by 354 rhizosphere $\left[\mathrm{O}_{2 \mathrm{r}}\right.$ ] [F6a] which is controlled by radial diffusion of $\mathrm{O}_{2}$ through soil water to 355 roots and nodules [F6b]. Soil water $\left[\mathrm{O}_{2}\right]$ is maintained by dissolution of $\mathrm{O}_{2}$ from soil air 356 which is in turn maintained by soil-atmosphere gas exchange and vertical diffusion 357 (Grant, 2004). $R_{\mathrm{h}}$ is first allocated to maintenance respiration $R_{\mathrm{m}}$ [F7-F8] and the 358 remainder if any is allocated to growth respiration $R_{\mathrm{g}}$ [F9]. If $R_{\mathrm{m}}$ exceeds $R_{\mathrm{h}}$, the shortfall 359 is made up from respiration of microbial protein $\mathrm{C}$, forcing senescence and litterfall of 360 associated non-protein C [F10-F11].

$361 \quad \mathbf{N}_{2}$ fixation

$362 \mathrm{~N}_{2}$ fixation $V_{\mathrm{N}_{2}}$ is driven by $R_{\mathrm{g}}$ [F12], but is constrained by accumulation of 363 nonstructural $\mathrm{N}\left(v_{\mathrm{n}}\right)$ with respect to nonstructural $\mathrm{C}$ and $\mathrm{P}$ also required for microbial 
364 growth in the nodule [F13]. $v_{\mathrm{n}}$ is the product of $V_{\mathrm{N}_{2}}$, so that [F12] simulates the inhibition 365 of $\mathrm{N}_{2}$ fixation by its product (Postgate, 1998). The value of $V_{\mathrm{N}_{2}}$ is also limited by the 366 additional $\mathrm{N}$ needed to maintain bacterial $\mathrm{N}$ content $\left[N_{\mathrm{n}}{ }^{\prime}\right]$ of $M_{\mathrm{n}}[\mathrm{F} 12]$, so that $\mathrm{N}_{2}$ fixation

367 is constrained by the need of nodule bacteria for $\mathrm{N}$ not met from other sources (Postgate, 368 1998). Respiration required for $\mathrm{N}_{2}$ fixation $R_{\mathrm{N}_{2}}$ [F14] is subtracted from $R_{\mathrm{g}}$ [F15] when 369 calculating microbial growth [F16-F18]. Microbial senescence drives N and P litterfall $370 \quad[\mathrm{~F} 19-\mathrm{F} 20]$.

\section{$371 \quad$ Nodule-root exchange}

372 Exchange of nonstructural $\mathrm{C}, \mathrm{N}$ and $\mathrm{P}$ between roots and nodules is driven by 373 concentration gradients [F21-F23] created by generation, transfer and consumption of 374 nonstructural $\mathrm{C}, \mathrm{N}$ and $\mathrm{P}$ in shoots, roots, mycorrhizae and nodules. Nonstructural $\mathrm{C}$ is 375 generated in shoots and transferred along concentration gradients to roots and thence to 376 nodules [F21]. Nonstructural $\mathrm{P}$ is generated in roots and transferred along concentration 377 gradients to shoots and nodules [F23]. Nonstructural $\mathrm{N}$ is generated in roots through 378 mineral uptake and in nodules through gaseous fixation [F22]. Nonstructural $\mathrm{C}, \mathrm{N}$ and $\mathrm{P}$ 379 in nodules is determined by root-nodule exchange, by nodule respiration and fixation, and 380 by remobilization from nodule litterfall [F24-F26].

381 Root nonstructural $\mathrm{N}\left(v_{r}\right)$ may rise if high mineral $\mathrm{N}$ concentrations in soil sustain 382 rapid $\mathrm{N}$ uptake by roots. Large $v_{\mathrm{r}}$ suppresses or even reverses the transfer of $v_{\mathrm{n}}$ from 383 nodule to root [F22], raising $v_{\mathrm{n}}[\mathrm{F} 25]$ and hence suppressing $V_{\mathrm{N}_{2}}$ [F12-F13]. Large $v_{r}$ 384 also accelerates the consumption of $\chi_{r}$, slowing its transfer to nodules [F21], reducing $385 \chi_{\mathrm{n}}[\mathrm{F} 24]$ and hence slowing nodule growth [F1]. Conversely, slow root $\mathrm{N}$ uptake 
caused by low soil mineral $\mathrm{N}$ concentrations would lower $v_{\mathrm{rt}}$ and raise $\chi_{\text {rt }}$, hastening the

387 transfer of $v_{\mathrm{n}}$ from nodule to root and of $\chi_{\mathrm{rt}}$ from root to nodule, lowering $\nu_{\mathrm{n}}$, raising $\chi_{\mathrm{n}}$,

388 and accelerating $V_{\mathrm{N}_{2}}$. However [F13] also allows $V_{\mathrm{N}_{2}}$ to be constrained by nonstructural C

389 and $\mathrm{P}$ concentrations arising from $\mathrm{CO}_{2}$ fixation and root $\mathrm{P}$ uptake.

390 Appendix G: $\mathbf{C H}_{\mathbf{4}}$ production and consumption

391 Anaerobic fermenters and $\mathbf{H}_{2}$ producing acetogens

392 The states $\mathrm{S}_{i, j, k}, \mathrm{~B}_{i, k}$ and $\mathrm{Z}_{i, j, k}$ in ecosys are substrates for hydrolysis by all active $(j$ $393=a$ ) heterotrophic biomass communities $\left(\mathrm{M}_{i, n, a}\right)$ [G1-G7] (Grant et al., 1993a), which 394 include fermenters plus acetogens. Hydrolysis products are transferred to soluble organic 395 matter $\left(\mathrm{DOC}_{i, k}\right)$ which is the substrate for respiration and uptake by microbial biomass $396\left(\mathrm{M}_{i, n, j}\right)$ as described for aerobic heterotrophs [G11] (Grant et al.,1993a). Respiration $\left(\mathrm{R}_{i, f}\right)$ 397 of $\mathrm{DOC}_{i, c}$ by fermenters plus acetogens $(n=f)$ is a Michaelis-Menten function of [DOC $\left.i, c\right]$ 398 inhibited by $\mathrm{O}_{2}$ [G1]. Respiration products are partitioned among $\mathrm{A}_{i, c}, \mathrm{CO}_{2}$ and $\mathrm{H}_{2}$ according 399 to Brock and Madigan (1991) [G2]. $\mathrm{R}_{i, f}$ beyond that used for maintenance respiration drives 400 the uptake of additional DOCi,c[G3] for microbial growth according to the growth yield (Y $f$ ) 401 from fermentation [G4]. The growth yield from fermentation is calculated by dividing the 402 free energy change of fermentation, adjusted for $\mathrm{H}_{2}$ product concentration [G5], by the 403 energy required to transform soluble organic $\mathrm{C}$ into microbial $\mathrm{C}$ [G4]. Change in $\mathrm{M}_{i, f, j}$ is 404 thus the difference between uptake and respiration of $\mathrm{DOC}_{i, c}$, less decomposition [G6]. This 405 change determines $\mathrm{M}_{i, f, a}$ used in the following calculation of $\mathrm{R}_{i, f}$ [G1]. Ratios of $\mathrm{M}_{i, f, j, c}$ to $406 \mathrm{M}_{i, f, j, n}$ determine mineralization-immobilization of $\mathrm{N}$ [G23] (Grant et al., 1993a). 407 Decomposition products $\left(\mathrm{D}_{i, f, j, k}\right)$ are partitioned to microbial residues $\left(\mathrm{Z}_{i, j, k}\right)$ and soil 
408 organic matter $\left(\mathrm{S}_{i, j, k}\right)$ (where $i=$ passive soil organic matter) [G26-G28] (Grant et al., 1993a)

409 which undergo further hydrolysis.

410 Acetotrophic methanogens

411 The fermenter product $\left(\mathrm{A}_{i, c}\right)$ [G2] is the substrate for respiration $\left(\mathrm{R}_{i, m}\right)$ by

412 acetotrophic methanogens $(n=m)$ [G7]. Respiration products are partitioned between $\mathrm{CH}_{4}$

413 and $\mathrm{CO}_{2}$ according to Brock and Madigan (1991) [G8]. $\mathrm{R}_{i, m}$ beyond that used for

414 maintenance respiration drives the uptake of additional Ai,c [G9] for microbial growth

415 according to the growth yield $\left(\mathrm{Y}_{m}\right)$ of acetotrophic methanogenesis [G10]. This growth yield

416 is calculated by dividing the free energy change of acetotrophic methanogenesis (Brock and

417 Madigan, 1991) by the energy required to transform acetate into microbial C. Acetogenic

418 methanogens in the model use acetate as their sole carbon and energy source (Smith and

419 Mah, 1980). Change in $\mathrm{M}_{i, m, j}$ is thus the difference between uptake and respiration of $\mathrm{A}_{i, c}$,

420 less decomposition [G11]. This change determines $\mathbf{M}_{i, m, a}$ used in the following calculation

421 of $\mathrm{R}_{i, m}$ [G7]. Mineralization and decomposition processes are the same as those for other

422 microbial populations.

423 Hydrogenotrophic methanogens

424 The fermenter products $\mathrm{CO}_{2}$ and $\mathrm{H}_{2}$ [G2] are the substrates for $\mathrm{CO}_{2}$ reduction by 425 hydrogenotrophic methanogens $(n=h)$ which are assumed to be autotrophic [G12]. 426 Respiration products are partitioned between $\mathrm{CH}_{4}$ and $\mathrm{H}_{2} \mathrm{O}$ according to Brock and Madigan 427 (1991) [G13]. $\mathrm{R}_{h}$ beyond that used for maintenance respiration drives the uptake of 428 additional $\mathrm{CO}_{2}$ [G14] for microbial growth according to the growth yield $\left(\mathrm{Y}_{h}\right)$ of 429 hydrogenotrophic methanogenesis (Brock and Madigan, 1991) [G15]. This growth yield is 430 calculated by dividing the free energy change of hydrogenotrophic methanogenesis, adjusted 
431 for $\mathrm{H}_{2}$ substrate concentration [G16], by the energy required to transform $\mathrm{CO}_{2}$ into microbial

432 C. Change in $\mathrm{M}_{h, j}$ is thus the difference between uptake and respiration of $\mathrm{CO}_{2}$, less

433 decomposition [G17]. This change determines $\mathrm{M}_{h, a}$ used in the following calculation of $\mathrm{R}_{h}$

434 [G12]. Mineralization and decomposition processes are the same as those for other microbial 435 populations.

436 Autotrophic methanotrophs

437 Methane generated by acetotrophic and hydrogenotrophic methanogens is the 438 substrate for $\mathrm{CH}_{4}$ oxidation by autotrophic methanotrophs $(n=t)$ [G18]. The stoichiometry 439 and energetics of the methanotrophic reactions [G22-G24] are based on those of $\mathrm{CH}_{4}$ to $\mathrm{CO}_{2}$ 440 in Brock and Madigan (1991). The oxidation of $\mathrm{CH}_{4}$ to $\mathrm{CO}_{2}$ is coupled through an energy 441 yield with the oxidation of $\mathrm{CH}_{4}$ to organic $\mathrm{C}$ used in microbial respiration [G19]. The energy 442 yield from $\mathrm{CH}_{4}$ oxidation is calculated by dividing the free energy change of $\mathrm{CH}_{4}$ oxidation 443 by the energy required to transform $\mathrm{CH}_{4}$ into organic $\mathrm{C}$ [G20]. Oxygen requirements to 444 sustain $\mathrm{CH}_{4}$ oxidation rates are then calculated from the stoichiometries of $\mathrm{CH}_{4}$ oxidation 445 [G22-G23] and aerobic microbial respiration [G24]. The $\mathrm{O}_{2}$ concentrations at 446 methanotrophic microsites are then found at which active $\mathrm{O}_{2}$ uptake driven by requirements 447 for $\mathrm{CH}_{4}$ oxidation equals spherical $\mathrm{O}_{2}$ diffusion to the microsites from the soil solution. 448 These microsites are considered to be uniformly distributed on soil surfaces and are separated 449 from the soil atmosphere (if present) by a water film of uniform thickness that depends upon 450 soil water potential. The $\mathrm{O}_{2}$ uptake by each aerobic microbial population in the model 451 competes with that by all other aerobic microbial populations (e.g. Grant and Rochette, 1994; 452 Grant, 1995], and is constrained by $\mathrm{O}_{2}$ transfer rates through the gaseous and aqueous phases 453 of the soil profile. The ratio of $\mathrm{O}_{2}$ uptake to $\mathrm{O}_{2}$ requirement $\left(f_{\mathrm{O}_{2}} t\right)$ is then used to constrain 
$454 \mathrm{CH}_{4}$ oxidation rates [G21] so that $\mathrm{CH}_{4}$ oxidation is stoichiometrically coupled to $\mathrm{O}_{2}$ uptake.

455 Growth respiration by methanotrophs is calculated as the difference between total respiration $456\left(\mathrm{R}_{t}\right)$ from eq. [G21b] and maintenance respiration $\left(\mathrm{R}_{\mathrm{m} t}\right)$ from eqs. [G18-G19] (Grant et al., 457 1993a). Growth respiration drives the uptake and transformation of additional $\mathrm{CH}_{4}$ into 458 microbial biomass $\left.\left(\mathrm{M}_{t, c}\right)[\mathrm{G} 25]\right)$ according to the growth yield. This yield is calculated by 459 dividing the free energy change of $\mathrm{CH}_{4}$ oxidation (Brock and Madigan, 1991) [G18] by the 460 energy required to construct new microbial biomass from $\mathrm{CH}_{4}$ [G26]. Net growth of the 461 methanotrophic population $\mathrm{M}_{t, j, c}$ is calculated as the uptake of $\mathrm{CH}_{4}-\mathrm{C}$ minus respiration and 462 decomposition of assimilated $\mathrm{C}$ [G27]. This change determines $\mathrm{M}_{t, a}$ used in the following 463 calculation of $X^{\prime} t$ [G18]). Mineralization and decomposition processes are the same as those 464 for other microbial populations.

465 This submodel of autotrophic methanotrophy has been used to simulate 466 methanotrophic growth yields, specific growth rates, $\mathrm{CH}_{4}$ concentration profiles and the 467 sensitivity of $\mathrm{CH}_{4}$ uptake to temperature and water content in soil columns (Grant, 1999). 468 The combined submodels of anaerobic fermentation, acetotrophic methanogenesis, 469 hydrogenotrophic methanogenesis and autotrophic methanotrophy have been used to 470 simulate methanogenic growth yields, specific growth rates, and the time course of $\mathrm{CH}_{4}$ 471 emissions from differently amended soil columns at different temperatures (Grant, 1998b).

472 All input parameter values used in eqs. [G1-G27] were derived from the microbiological 473 literature and remain unchanged from those used in Grant (1998b) and in Grant (1999).

474 Appendix H: Inorganic $\mathbf{N}$ transformations

475 Mineralization and immobilization of $\mathrm{NH}_{4}{ }^{+}$by all microbial populations 
Each functional component $(j)(j=$ labile or resistant $)$ of each microbial 477 population $(m)(m=$ obligately aerobic bacteria, obligately aerobic fungi, facultatively 478 anaerobic denitrifiers, anaerobic fermenters plus $\mathrm{H}_{2}$-producing acetogens, acetotrophic 479 methanogens, hydrogenotrophic methanogens and methanotrophs, $\mathrm{NH}_{4}{ }^{+}$and $\mathrm{NO}_{2}{ }^{-}$ 480 oxidizers, and non-symbiotic diazotrophs) in each substrate-microbe complex $(i)(i=$ 481 animal manure, coarse woody plant residue, fine non-woody plant residue, particulate 482 organic matter, or humus) seeks to maintain a set C:N ratio by mineralizing $\mathrm{NH}_{4}{ }^{+}[\mathrm{H} 1 \mathrm{a}]$ 483 or by immobilizing $\mathrm{NH}_{4}^{+}[\mathrm{H} 1 \mathrm{~b}]$ or $\mathrm{NO}_{3}{ }^{-}$[H1c]. Provision is made for C:N ratios to rise 484 above set values during immobilization, but at a cost to microbial function. These 485 transformations control the exchange of $\mathrm{N}$ between organic and inorganic states.

\section{Oxidation of DOC and reduction of $\mathbf{O}_{2}$ by heterotrophs}

487 Constraints on heterotrophic oxidation of DOC imposed by $\mathrm{O}_{2}$ uptake are solved 488 in four steps:

489 1) DOC oxidation under non-limiting $\mathrm{O}_{2}$ is calculated from active biomass and DOC 490 concentration $[\mathrm{H} 2]$,

491 2) $\mathrm{O}_{2}$ reduction under non-limiting $\mathrm{O}_{2}$ is calculated from 1) using a set respiratory 492 quotient [H3],

493 3) $\mathrm{O}_{2}$ reduction under ambient $\mathrm{O}_{2}$ is calculated from radial $\mathrm{O}_{2}$ diffusion through water 494 films of thickness determined by soil water potential [H4a] coupled with active uptake 495 at heterotroph surfaces driven by 2) [H4b]. $\mathrm{O}_{2}$ diffusion and active uptake is 496 population-specific, allowing the development of more anaerobic conditions at 497 microbial surfaces associated with more biologically active substrates. $\mathrm{O}_{2}$ uptake by 
498 heterotrophs also accounts for competition with $\mathrm{O}_{2}$ uptake by nitrifiers, roots and 499 mycorrhizae,

500 4) DOC oxidation under ambient $\mathrm{O}_{2}$ is calculated from 2) and 3) [H5]. The energy yield 501 of DOC oxidation drives the uptake of additional DOC for construction of microbial 502 biomass $\left(M_{i, h}\right)$ according to construction energy costs of each heterotrophic population 503 [H7-H13] in Grant and Pattey (2003). Energy costs of denitrifiers are slightly larger 504 than those of obligate heterotrophs, placing denitrifiers at a competitive disadvantage 505 for growth and hence DOC oxidation if electron acceptors other than $\mathrm{O}_{2}$ are not used.

506 Oxidation of DOC and reduction of $\mathrm{NO}_{3}^{-}, \mathrm{NO}_{2}{ }^{-}$and $\mathrm{N}_{2} \mathrm{O}$ by denitrifiers

507 Constraints imposed by $\mathrm{NO}_{3}^{-}$availability on DOC oxidation by denitrifiers are 508 solved in five steps:

509 1) $\mathrm{NO}_{3}{ }^{-}$reduction under non-limiting $\mathrm{NO}_{3}{ }^{-}$is calculated from a fraction of electrons 510 demanded by DOC oxidation but not accepted by $\mathrm{O}_{2}$ because of diffusion limitations $511 \quad[\mathrm{H} 6]$,

512 2) $\mathrm{NO}_{3}{ }^{-}$reduction under ambient $\mathrm{NO}_{3}{ }^{-}$is calculated from 1) [H7],

513 3) $\mathrm{NO}_{2}^{-}$reduction under ambient $\mathrm{NO}_{2}{ }^{-}$is calculated from demand for electrons not met by $514 \quad \mathrm{NO}_{3}^{-}$in 2) [H8],

515 4) $\mathrm{NO}_{2}^{-}$reduction under ambient $\mathrm{NO}_{2}{ }^{-}$is calculated from demand for electrons not met by $516 \quad \mathrm{NO}_{2}^{-}$in 3) [H9],

517 5) additional DOC oxidation enabled by $\mathrm{NO}_{\mathrm{x}}$ reduction in 2), 3) and 4) is added to that 518 enabled by $\mathrm{O}_{2}$ reduction from [H5], the energy yield of which drives additional DOC 519 uptake for construction of $M_{i, n}$. This additional uptake offsets the disadvantage 520 incurred by the larger construction energy costs of denitrifiers. 


\section{Oxidation of $\mathrm{NH}_{3}$ and reduction of $\mathrm{O}_{2}$ by nitrifiers}

522 Constraints on nitrifier oxidation of $\mathrm{NH}_{3}$ imposed by $\mathrm{O}_{2}$ uptake are solved in four 523 steps:

524 1) substrate $\left(\mathrm{NH}_{3}\right)$ oxidation under non-limiting $\mathrm{O}_{2}$ is calculated from active biomass and 525 from $\mathrm{NH}_{3}$ and $\mathrm{CO}_{2}$ concentrations [H11],

526 2) $\mathrm{O}_{2}$ reduction under non-limiting $\mathrm{O}_{2}$ is calculated from 1) using set respiratory 527 quotients [H12],

528 3) $\mathrm{O}_{2}$ reduction under ambient $\mathrm{O}_{2}$ is calculated from radial $\mathrm{O}_{2}$ diffusion through water 529 films of thickness determined by soil water potential [H13a] coupled with active 530 uptake at nitrifier surfaces driven by 2) [H13b]. $\mathrm{O}_{2}$ uptake by nitrifiers also accounts 531 for competition with $\mathrm{O}_{2}$ uptake by heterotrophic DOC oxidizers, roots and 532 mycorrhizae,

533 4) $\mathrm{NH}_{3}$ oxidation under ambient $\mathrm{O}_{2}$ is calculated from 2) and 3) [H14]. The energy yield 534 of $\mathrm{NH}_{3}$ oxidation drives the fixation of $\mathrm{CO}_{2}$ for construction of $M_{i, n}$ according to 535 construction energy costs of each nitrifier population [H32-H34] in Grant and Pattey $536 \quad(2003)$

537 Oxidation of $\mathrm{NO}_{2}^{-}$and reduction of $\mathrm{O}_{2}$ by nitrifiers

538 Constraints on nitrifier oxidation of $\mathrm{NO}_{2}^{-}$imposed by $\mathrm{O}_{2}$ uptake [H15-H18] are 539 solved in the same way as are those of $\mathrm{NH}_{3}$ [H11-H14]. The energy yield of $\mathrm{NO}_{2}{ }^{-}$ 540 oxidation drives the fixation of $\mathrm{CO}_{2}$ for construction of $M_{i, o}$ according to construction 541 energy costs of each nitrifier population.

542 Oxidation of $\mathrm{NH}_{3}$ and reduction of $\mathrm{NO}_{2}{ }^{-}$by nitrifiers 
543 Constraints on nitrifier oxidation imposed by $\mathrm{NO}_{2}{ }^{-}$availability are solved in three 544 steps:

545 1) $\mathrm{NO}_{2}^{-}$reduction under non-limiting $\mathrm{NO}_{2}^{-}$is calculated from a fraction of electrons 546 demanded by $\mathrm{NH}_{3}$ oxidation but not accepted by $\mathrm{O}_{2}$ because of diffusion limitations $547 \quad[\mathrm{H} 19]$,

548 2) $\mathrm{NO}_{2}^{-}$reduction under ambient $\mathrm{NO}_{2}^{-}$and $\mathrm{CO}_{2}$ is calculated from step (1) [H20], $549 \quad$ competing for $\mathrm{NO}_{2}^{-}$with [H18],

550 3) additional $\mathrm{NH}_{3}$ oxidation enabled by $\mathrm{NO}_{2}{ }^{-}$reduction in 2) [H21] is added to that 551 enabled by $\mathrm{O}_{2}$ reduction from [H14]. The energy yield from this oxidation drives the 552 fixation of additional $\mathrm{CO}_{2}$ for construction of $M_{i, n}$. 


\title{
Equations and definitions of variables
}

\section{Appendix A: Soil C, $\mathbf{N}$ and $\mathbf{P}$ transformations}

\author{
Decomposition \\ $D_{S i, j, l, \mathrm{C}}=D_{S i, j, l, \mathrm{C}}^{\prime} M_{i, d, l, \mathrm{C}} f_{\mathrm{tg} l}\left(S_{i, l, \mathrm{C}} / G_{i, l, \mathrm{C}}\right)$ \\ $D_{Z i, j, l, \mathrm{C}}=D_{Z i, j, l, \mathrm{C}}^{\prime} M_{i, d, l, \mathrm{C}} f_{\mathrm{tg} l}\left(Z_{i, l, \mathrm{C}} / G_{i, l, \mathrm{C}}\right)$ \\ $D_{A i, l, \mathrm{C}}=D_{A i, l, \mathrm{C}}^{\prime} M_{i, d, l, \mathrm{C}} f_{\mathrm{tg} l}\left(A_{i, l, \mathrm{C}} / G_{i, l, \mathrm{C}}\right)$ \\ $S_{i, l, \mathrm{C}}=\Sigma_{j} S_{i, j, l, \mathrm{C}}$ \\ $Z_{i, l, \mathrm{C}}=\Sigma_{j} Z_{i, j, l, \mathrm{C}}$ \\ $G_{i, l, \mathrm{C}}=S_{i, l, \mathrm{C}}+Z_{i, l, \mathrm{C}}+A_{i, l, \mathrm{C}}$
}

total $\mathrm{C}$ in all kinetic components

of litter, POC, humus

total $\mathrm{C}$ in all kinetic components

[A2b]

of microbial residues

total $\mathrm{C}$ in substrate-microbe

complexes 
$M_{i, d, l, \mathrm{C}}=M_{i, a, l, \mathrm{C}+} \boldsymbol{q}_{\boldsymbol{m}}\left(M_{i, a, l, \mathrm{C}} G_{i x, l, \mathrm{C}}-M_{i x, a, l, \mathrm{C}} G_{i, l, \mathrm{C}}\right) /\left(G_{i x, l, \mathrm{C}}+G_{i, l, \mathrm{C}}\right)$

$M_{i, a, l, \mathrm{C}}=\Sigma_{n} M_{i, n, a, l \mathrm{C}}$

$D_{S i, j, l, \mathrm{C}}^{\prime}=\left\{\boldsymbol{D}_{S j, \mathrm{C}}\left[S_{i, j, l, \mathrm{C}}\right]\right\} /\left\{\left[S_{i, j, l, \mathrm{C}}\right]+\boldsymbol{K}_{\mathbf{m} \boldsymbol{D}}\left(1.0+\left[\Sigma M_{i, d, l, \mathrm{C}}\right] / \boldsymbol{K}_{\mathbf{i} \boldsymbol{D}}\right)\right\}$

$D_{Z i, j, l, \mathrm{C}}^{\prime}=\left\{\boldsymbol{D}_{Z j, \mathrm{C}}\left[Z_{i, j, l, \mathrm{C}}\right]\right\} /\left\{\left[Z_{i, j, l, \mathrm{C}}\right]+\boldsymbol{K}_{\mathbf{m} \boldsymbol{D}}\left(1.0+\left[M_{i, d, l, \mathrm{C}}\right] / \boldsymbol{K}_{\mathbf{i} \boldsymbol{D}}\right)\right\}$

$D_{A i, l, \mathrm{C}}^{\prime}=\left\{\boldsymbol{D}_{A, \mathrm{C}}\left[A_{i, l, \mathrm{C}}\right]\right\} /\left\{\left[A_{i, l, \mathrm{C}}\right]+\boldsymbol{K}_{\mathbf{m} \boldsymbol{D}}\left(1.0+\left[M_{i, d, l, \mathrm{C}}\right] / \boldsymbol{K}_{\mathbf{i} \boldsymbol{D}}\right)\right\}$

$\delta S_{i, j, k, l, \mathrm{C}} / \delta t=\beta \Sigma_{n}\left(U_{i, n, l \mathrm{C}}-R_{\mathrm{h} i, n, l}\right)\left(S_{i, j, k, l, \mathrm{C}}^{\prime} / S_{i, j, l, \mathrm{C}}^{\prime}\right)\left\{\left(S_{i, j, l, \mathrm{C}}^{\prime} / S_{i, j, l, \mathrm{C}}\right) /\left(S_{i, j, l, \mathrm{C}}^{\prime} / S_{i, j, l, \mathrm{C}}+\boldsymbol{K}_{\mathrm{iS}}\right)\right\}$

$f_{\mathrm{tg} l}=T_{\mathrm{s} l}\left\{e^{\left[B-\boldsymbol{H}_{\mathbf{a}} /\left(\boldsymbol{R} T_{\mathrm{s}}\right)\right]}\right\} /\left\{1+e^{\left[\left(\boldsymbol{H}_{\mathbf{d} \mathbf{l}}-\boldsymbol{S} T_{\mathrm{s}}\right) /\left(\boldsymbol{R} T_{\mathrm{s} l}\right)\right]}+e^{\left[\left(\boldsymbol{S} T_{\mathrm{s} l}-\boldsymbol{H}_{\mathbf{d h}}\right) /\left(\boldsymbol{R} T_{\mathrm{s} l}\right)\right]}\right\}$ redistribution of active microbial

[A3a]

biomass from each substrate-

microbe complex $i$ to other

[A3b]

substrate-microbe complexes ix

according to concentration

differences (priming)

substrate and water constraint on

[A4a]

$D$ from colonized litter, POC and

humus, microbial residues and

[A4b]

adsorbed SOC

[A4c]

colonized litter determined by

microbial growth into uncolonized

litter

Arrhenius function for $D$ and $R_{\mathrm{h}}$ 


\section{DON, DOP}

$\left(Y_{i, L C}<0\right) \quad$ desorption of

DON, DOP

\section{Microbial growth}

$R_{\mathrm{h}}=\Sigma_{i} \Sigma_{n} \Sigma_{l} R_{\mathrm{h} i, n, l}$ 


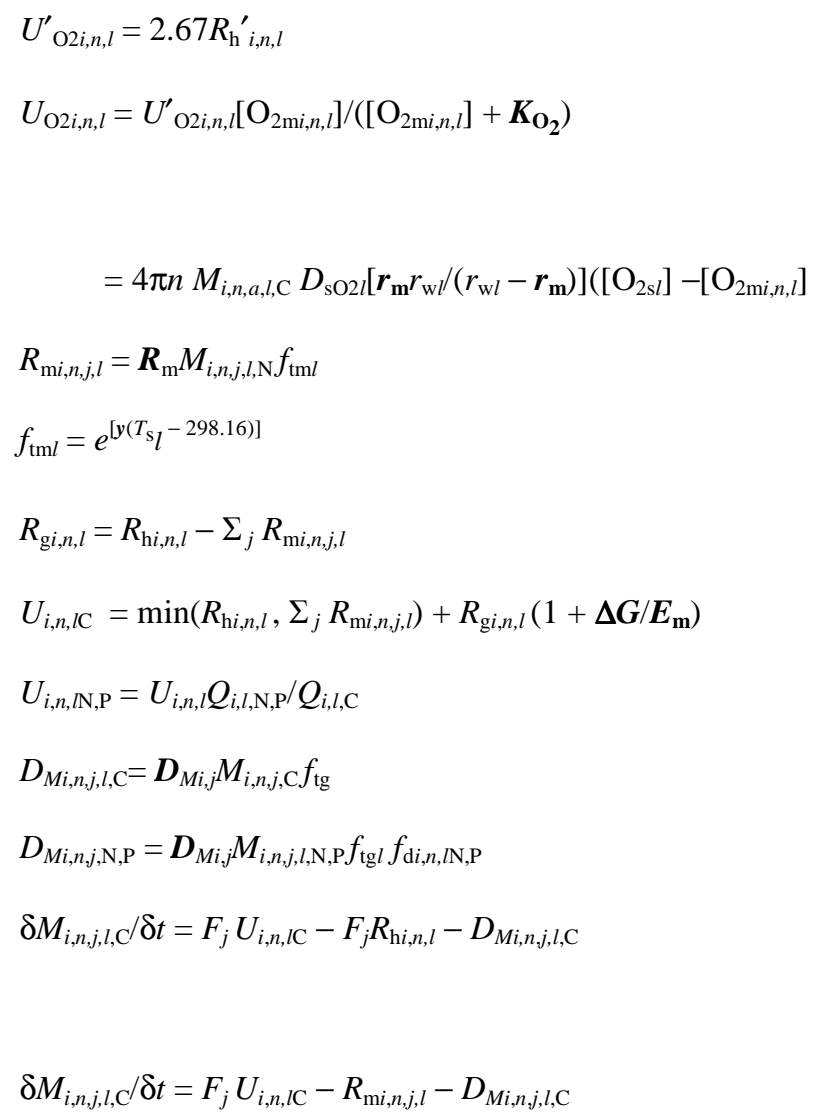

\section{Microbial nutrient exchange}


$U_{\mathrm{NH} 4 i, n, j, l}=\left(M_{i, n, j, l, C} C_{\mathrm{N} j}-M_{i, n, j, l, N}\right)$

$U_{\mathrm{NH}_{4} i, n, j, l}=\min \left\{\left(M_{i, n, j, l, C} C_{\mathrm{N} j}-M_{i, n, j, l, N}\right)\right.$,

$\left.\boldsymbol{U}^{{ }^{\prime} \mathbf{N H}_{\mathbf{4}}} a_{i, n, j, l}\left(\left[\mathrm{NH}_{4}{ }_{i, n, j, l}^{+}\right]-\left[\mathbf{N H}_{\mathbf{4}}{ }_{\mathbf{m n}}\right]\right) /\left(\left[\mathrm{NH}_{4}{ }_{i, n, j, l}{ }^{+}\right]-\left[\mathbf{N H}_{\mathbf{4}}{ }_{\mathbf{m n}}^{+}\right]+\boldsymbol{K}_{\mathbf{N H}_{\mathbf{4}}}\right)\right\}$

$U_{\mathrm{NO}_{3} i, n, j, l}=\min \left\{\left(M_{i, n, j, l, C} \boldsymbol{C}_{\mathrm{N} j}-\left(M_{i, n, j, l, N}+U_{\mathrm{NH}_{4} i, n, j, l}\right)\right)\right.$

$\left.\boldsymbol{U}^{\prime} \mathbf{N O}_{\mathbf{3}} a_{i, n, j, l}\left(\left[\mathrm{NO}_{3}{ }_{i, n, j, l}^{-}\right]-\left[\mathbf{N O}_{\mathbf{3}}{ }_{\mathbf{~ m n}}\right]\right) /\left(\left[\mathrm{NO}_{3}^{-}{ }_{i, n, j, l}^{-}\right]-\left[\mathbf{N O}_{\mathbf{3}}^{-}{ }_{\mathbf{~ m n}}\right]+\boldsymbol{K}_{\mathbf{N O}_{\mathbf{3}}}\right)\right\}$

$U_{\mathrm{PO} 4 i, n, j, l}=\left(M_{i, n, j, l, C} \boldsymbol{C}_{\mathbf{P} j}-M_{i, n, j, l, P}\right)$

$U_{\mathrm{PO} 4 i, n, j, l}=\min \left\{\left(M_{i, n, j, l, C} \boldsymbol{C}_{\mathbf{P} j}-M_{i, n, j, l, P}\right)\right.$

$\left.\boldsymbol{U}^{\prime} \mathbf{P O}_{\mathbf{4}} A_{i, n, j, l}\left(\left[\mathrm{H}_{2} \mathrm{PO}_{4}^{-}{ }_{i, n, j, l}^{-}\right]-\left[\mathbf{H}_{\mathbf{2}} \mathbf{P O}_{4}^{-}{ }_{\mathbf{m n}}\right]\right) /\left(\left[\mathrm{H}_{2} \mathrm{PO}_{4}^{-}{ }_{i, n, j, l}\right]-\left[\mathbf{H}_{\mathbf{2}} \mathbf{P O}_{\mathbf{4}}^{-}{ }_{\mathbf{m n}}\right]+\boldsymbol{K}_{\mathbf{P O}_{\mathbf{4}}}\right)\right\}$

$\Phi_{i, n=f, j, l}=\max \left\{0, M_{i, n=f, j, l, \mathrm{C}} \boldsymbol{C}_{\mathbf{N} j}-M_{i, n=f, j, l, \mathrm{~N}}-\max \left\{0, U_{i, n=f, j, l, \mathrm{~N}}\right\}\right\}$

$R_{\Phi, n=f, j, l}=\boldsymbol{E}_{\boldsymbol{\Phi}} \Phi_{i, n=f, j, l}$

$$
U_{\mathrm{NH}_{4}}<0 \quad \text { mineralization }
$$

immobilizatio diazotrophic population 
$\delta M_{i, n, j, l, \mathrm{~N}} / \delta t=F_{j} U_{i, n, l, \mathrm{~N}}+U_{\mathrm{NH}_{4 i, n, j, l}}+U_{\mathrm{NO}_{3 i, n, j, l}}+\Phi_{i, n=f, j, l}-D_{M i, n, j, l, \mathrm{~N}}$

$\delta M_{i, n, j, l, \mathrm{P}} / \delta t=F_{j} U_{i, n, l, \mathrm{P}}+U_{\mathrm{PO}_{4 i, n, j, l}}-D_{M i, n, j, l, \mathrm{p}}$

$M_{i, n, a, l, \mathrm{C}}=M_{i, n, j=l a b i l e, l, \mathrm{C}}+M_{i, n, j=\text { resistant }, l, \mathrm{C}} F_{\mathrm{r}} / F_{1}$
[A29b]

[A30a]

\section{Humification}

$H_{S i, j=\text { lignin }, l, \mathrm{C}}=D_{S i, j=\text { lignin }, l, \mathrm{C}}$

decomposition products of litter

[A31]

$H_{S i, j=\text { lignin, }, \mathrm{N}, \mathrm{P}}=D_{S i, j=\operatorname{lignin}, l, \mathrm{~N}, \mathrm{P}}$

added to POC depending on lignin

[A32]

$H_{S i, j \neq l \operatorname{lignin}, l, \mathrm{C}}=H_{S i, j=\operatorname{lignin}, l, \mathrm{C}} \boldsymbol{L}_{\mathbf{h} j}$

[A33]

$H_{S i, j \neq l \operatorname{lignin}, l, \mathrm{~N}, \mathrm{P}}=H_{S i, j \neq l i g n i n, l, \mathrm{C}} S_{i, l, \mathrm{~N}, \mathrm{P}} / S_{i, l, \mathrm{C}}$

[A34]

$H_{M i, n, j, l, \mathrm{C}}=D_{M i, n, j, l, \mathrm{C}} \boldsymbol{F}_{\mathbf{h}}$

decomposition products of

[A35]

$H_{M i, n, j, l, \mathrm{~N}, \mathrm{P}}=H_{M i, n, j, l, \mathrm{C}} M_{i, n, j, l, \mathrm{~N}, \mathrm{P}} / M_{i, n, j, l, \mathrm{C}}$

\section{Definition of variables in appendix A}

depending on clay

\begin{tabular}{lcccc}
\hline Variable Definition & Unit & Equation & Reference \\
\hline
\end{tabular}


woody litter, POC, humus

kinetic component: labile $l$, resistant $r$, active $a$

soil or litter layer

microbial functional type: heterotrophic (bacteria, fungi),

autotrophic (nitrifiers, methanotrophs), diazotrophic, obligate

aerobe, facultative anaerobes (denitrifiers), obligate anaerobes

(methanogens)

$\begin{array}{ll}A_{i, l, \mathrm{C}} & \text { mass of adsorbed SOC } \\ {\left[A_{i, l, \mathrm{C}}\right]} & \text { concentration of adsorbed SOC in soil } \\ a & \text { microbial surface area } \\ \boldsymbol{B} & \text { parameter such that } f_{\mathrm{tg}}=1.0 \text { at } T_{l}=298.15 \mathrm{~K} \\ \boldsymbol{b} & \text { Freundlich exponent for sorption isotherm } \\ & \\ \boldsymbol{\beta} & \text { specific colonization rate of uncolonized substrate }\end{array}$

Variables

$C_{\mathrm{N}, \mathrm{P} i, n, a, l}$

ratio of $M_{i, n, a, \mathrm{~N}, \mathrm{P}}$ to $M_{i, n, a, \mathrm{C}}$

g N or $\mathrm{P} \mathrm{g} \mathrm{C}^{-1}$

$[\mathrm{A} 1 \mathrm{c}, \mathrm{A} 2 \mathrm{c}]$

[A4c]

[A26]

[A6]

26.230

[A8]

Grant et al.

(1993a, b)

[A5]

Grant et al. 


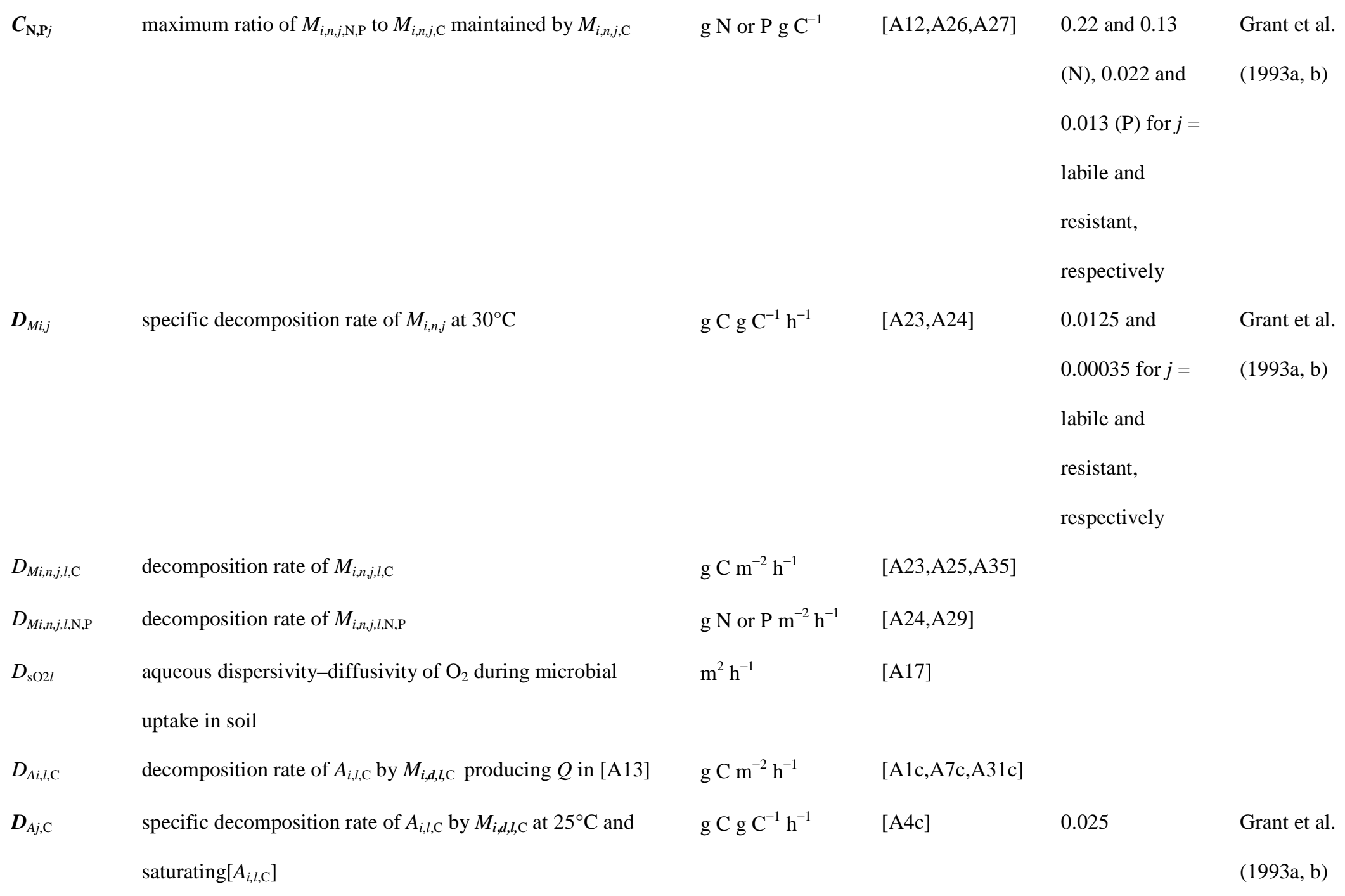




\begin{tabular}{|c|c|c|c|c|c|}
\hline$D_{A i, j, l, \mathrm{~N}, \mathrm{P}}$ & decomposition rate of $A_{i, l, \mathrm{~N}, \mathrm{P}}$ by $M_{i, l, l, \mathrm{C}}$ & $\mathrm{g} \mathrm{N}$ or $\mathrm{P} \mathrm{m}^{-2} \mathrm{~h}^{-1}$ & {$[\mathrm{~A} 7 \mathrm{c}]$} & & \\
\hline$D_{A i, j, l, \mathrm{C}}^{\prime}$ & specific decomposition rate of $S_{i, j, l, \mathrm{C}}$ by $\Sigma_{n} M_{i, n, a, l}$ at $25^{\circ} \mathrm{C}$ & $\mathrm{g} \mathrm{Cg} \mathrm{C}^{-1} \mathrm{~h}^{-1}$ & {$[\mathrm{~A} 1 \mathrm{a}, \mathrm{A} 4 \mathrm{c}]$} & & \\
\hline$D_{S i, j, l, \mathrm{C}}$ & decomposition rate of $S_{i, j, l, \mathrm{C}}$ by $\Sigma_{n} M_{i, n, a, l}$ producing $Q$ in [A13] & $\mathrm{g} \mathrm{C} \mathrm{m}^{-2} \mathrm{~h}^{-1}$ & {$[\mathrm{~A} 1 \mathrm{a}, \mathrm{A} 7 \mathrm{a}, \mathrm{A} 31 \mathrm{a}]$} & & \\
\hline $\boldsymbol{D}_{S j, \mathrm{C}}$ & $\begin{array}{l}\text { specific decomposition rate of } S_{i, j, l, \mathrm{C}} \text { by } \Sigma_{n} M_{i, n, a, l} \text { at } 25^{\circ} \mathrm{C} \text { and } \\
\text { saturating }\left[S_{i, l, \mathrm{C}}\right]\end{array}$ & $\mathrm{g} \mathrm{Cg} \mathrm{C}^{-1} \mathrm{~h}^{-1}$ & [A4a] & $\begin{array}{l}1.0,1.0,0.15, \text { and } \\
0.025 \text { for } j= \\
\text { protein, } \\
\text { carbohydrate, } \\
\text { cellulose, and } \\
\text { lignin }\end{array}$ & $\begin{array}{l}\text { Grant et al. } \\
(1993 a, b)\end{array}$ \\
\hline$D_{S i, j, l, \mathrm{~N}, \mathrm{P}}$ & decomposition rate of $S_{i, j, l, \mathrm{~N}, \mathrm{P}}$ by $\Sigma_{n} M_{i, n, a, l}$ & $\mathrm{~g} \mathrm{~N}$ or $\mathrm{P} \mathrm{m}^{-2} \mathrm{~h}^{-1}$ & {$[\mathrm{~A} 7 \mathrm{a}, \mathrm{A} 32]$} & & \\
\hline$D^{\prime}{ }_{S i, j, l, \mathrm{C}}$ & specific decomposition rate of $S_{i, j, l, \mathrm{C}}$ by $\Sigma_{n} M_{i, n, a, l}$ at $25^{\circ} \mathrm{C}$ & $\mathrm{g} \mathrm{C} \mathrm{g} \mathrm{C}^{-1} \mathrm{~h}^{-1}$ & {$[\mathrm{~A} 1 \mathrm{a}, \mathrm{A} 4 \mathrm{a}]$} & & \\
\hline$D_{Z i, j, l, \mathrm{C}}$ & decomposition rate of $Z_{i, j, l, \mathrm{C}}$ by $\Sigma_{n} M_{i, n, a, l}$ producing $Q$ in [A13] & $\mathrm{g} \mathrm{C} \mathrm{m}^{-2} \mathrm{~h}^{-1}$ & {$[\mathrm{~A} 1 \mathrm{~b}, \mathrm{~A} 7 \mathrm{~b}]$} & & \\
\hline$D_{Z i, j, \mathrm{~N}, \mathrm{P}}$ & decomposition rate of $Z_{i, j, l, \mathrm{~N}, \mathrm{P}}$ by $\Sigma_{n} M_{i, n, a, l}$ & $\mathrm{~g} \mathrm{~N}$ or $\mathrm{P} \mathrm{m}^{-2} \mathrm{~h}^{-1}$ & {$[\mathrm{~A} 7 \mathrm{~b}]$} & & \\
\hline $\boldsymbol{D}_{Z j, \mathrm{C}}$ & $\begin{array}{l}\text { specific decomposition rate of } Z_{i, j, l, \mathrm{C}} \text { by } \Sigma_{n} M_{i, n, a, l} \text { at } 25^{\circ} \mathrm{C} \text { and } \\
\text { saturating }\left[Z_{i, l, \mathrm{C}}\right]\end{array}$ & $\mathrm{gC} \mathrm{g} \mathrm{C}^{-1} \mathrm{~h}^{-1}$ & {$[\mathrm{~A} 4 \mathrm{~b}]$} & $\begin{array}{l}0.25 \text { and } 0.05 \text { for } \\
j=\text { labile and } \\
\text { resistant biomass }\end{array}$ & $\begin{array}{l}\text { Grant et al. } \\
(1993 a, b)\end{array}$ \\
\hline$D^{\prime} z_{i j, l, l, C}$ & specific decomposition rate of $Z_{i, j, l, \mathrm{C}}$ by $\Sigma_{n} M_{i, n, a, l}$ at $25^{\circ} \mathrm{C}$ & $\mathrm{gC} \mathrm{g} \mathrm{C}^{-1} \mathrm{~h}^{-1}$ & {$[\mathrm{~A} 1 \mathrm{~b}, \mathrm{~A} 4 \mathrm{~b}]$} & & \\
\hline$\Delta G$ & energy yield of $\mathrm{C}$ oxidation and $\mathrm{O}_{2}$ reduction & $\mathrm{kJ} \mathrm{g} \mathrm{C}^{-1}$ & [A21] & 37.5 & \\
\hline
\end{tabular}




\begin{tabular}{|c|c|c|c|c|c|}
\hline $\boldsymbol{E}_{\mathrm{m}}$ & energy requirement for growth of $M_{i, n, a, l}$ & $\mathrm{~kJ} \mathrm{~g} \mathrm{C}^{-1}$ & [A21] & 25 & \\
\hline \multirow[t]{2}{*}{$\boldsymbol{E}_{\boldsymbol{\Phi}}$} & energy requirement for non-symbiotic $\mathrm{N}_{2}$ fixation by & $\mathrm{g} \mathrm{C} \mathrm{g} \mathrm{N}^{-1}$ & [A28] & 5 & Waring and \\
\hline & heterotrophic diazotrophs $(n=f)$ & & & & Running (1998) \\
\hline \multirow[t]{2}{*}{$F_{\mathrm{h}}$} & fraction of products from microbial decomposition that are & & [A35] & $0.167+$ & \\
\hline & humified (function of clay content) & & & $0.167 *$ clay & \\
\hline \multirow[t]{2}{*}{$F_{1}$} & fraction of microbial growth allocated to labile component & & {$[\mathrm{A} 25, \mathrm{~A} 29, \mathrm{~A} 30]$} & 0.55 & Grant et al. \\
\hline & $M_{i, n, l}$ & & & & $(1993 a, b)$ \\
\hline \multirow[t]{2}{*}{$F_{\mathbf{r}}$} & fraction of microbial growth allocated to resistant component & & {$[\mathrm{A} 25, \mathrm{~A} 29, \mathrm{~A} 30]$} & 0.45 & Grant et al. \\
\hline & $M_{i, n, r}$ & & & & $(1993 a, b)$ \\
\hline$F_{\mathrm{s}}$ & equilibrium ratio between $Q_{i, l, \mathrm{C}}$ and $H_{i, l, \mathrm{C}}$ & & [A8] & & \\
\hline \multirow[t]{4}{*}{$f_{\mathrm{di}, n, \mathbb{N}, \mathrm{P}}$} & fraction of $\mathrm{N}$ or P released with $D_{M i, n, j, l, \mathrm{C}}$ during & dimensionless & [A24] & $0.33 U_{\mathrm{NH} 4}>0$ & \\
\hline & decomposition & & & $1.00 U_{\mathrm{NH} 4}<0$ & \\
\hline & & & & $0.33 U_{\mathrm{PO} 4}>0$ & \\
\hline & & & & $1.00 U_{\mathrm{PO} 4}<0$ & \\
\hline \multirow[t]{2}{*}{$f_{\mathrm{tg} l}$} & temperature function for microbial growth respiration & dimensionless & {$[\mathrm{A} 1, \mathrm{~A} 6, \mathrm{~A} 13$,} & & \\
\hline & & & $\mathrm{A} 23, \mathrm{~A} 24]$ & & \\
\hline$f_{\mathrm{tm} l}$ & temperature function for maintenance respiration & dimensionless & {$[\mathrm{A} 18, \mathrm{~A} 19]$} & & \\
\hline
\end{tabular}




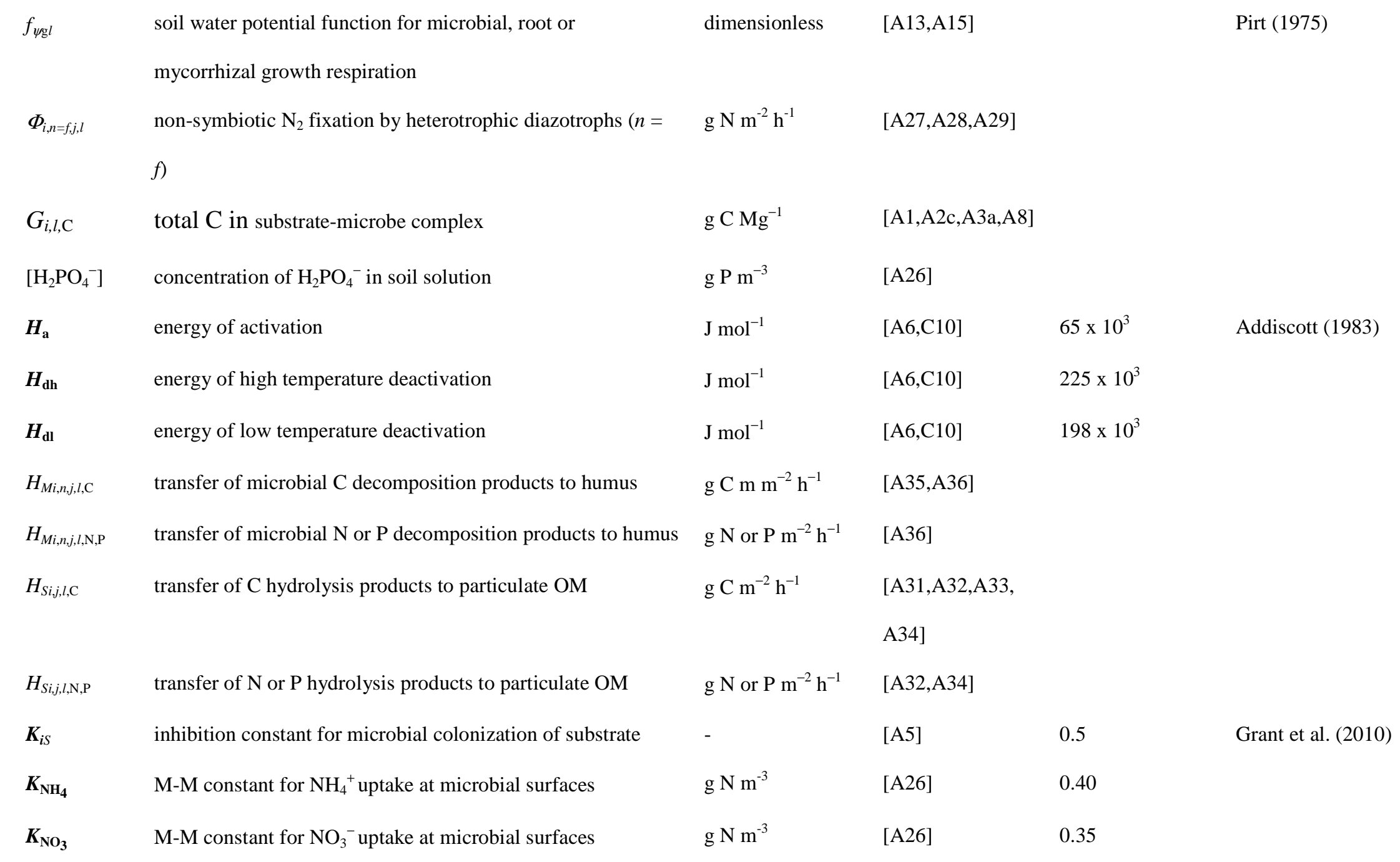




\begin{tabular}{|c|c|c|c|c|c|}
\hline$K_{\mathrm{PO}_{4}}$ & M-M constant for $\mathrm{H}_{2} \mathrm{PO}_{4}{ }^{-}$uptake at microbial surfaces & $\mathrm{g} \mathrm{P} \mathrm{m}^{-3}$ & {$[\mathrm{~A} 26]$} & 0.125 & \\
\hline$K_{\mathrm{i} D}$ & inhibition constant for $\left[M_{i, n, a}\right]$ on $S_{i, \mathrm{C}}, Z_{i, \mathrm{C}}$ & $\mathrm{g} \mathrm{C} \mathrm{m}^{-3}$ & {$[\mathrm{~A} 4]$} & 25 & Lizama and \\
\hline$K_{\mathrm{m} D}$ & Michaelis-Menten constant for $D_{S i, j, \mathrm{C}}$ & $\mathrm{g} \mathrm{C} \mathrm{Mg}^{-1}$ & {$[\mathrm{~A} 4]$} & 75 & Suzuki (1990), \\
\hline$K_{\mathrm{m} Q_{\mathbf{C}}}$ & Michaelis-Menten constant for $R_{\mathrm{h}, n}^{\prime}$ on $\left[Q_{i, \mathrm{C}}\right]$ & $\mathrm{g} \mathrm{C} \mathrm{m}^{-3}$ & [A13] & 36 & $\begin{array}{l}\text { Grant et al. } \\
(1993 a, b)\end{array}$ \\
\hline$K_{\mathrm{O}_{2}}$ & $\begin{array}{l}\text { Michaelis-Menten constant for reduction of } \mathrm{O}_{2 \mathrm{~s}} \text { by microbes, } \\
\text { roots and mycorrhizae }\end{array}$ & $\mathrm{g} \mathrm{O}_{2} \mathrm{~m}^{-3}$ & [A17] & 0.064 & Griffin (1972) \\
\hline$k_{\mathrm{ts}}$ & equilibrium rate constant for sorption & $\mathrm{h}^{-1}$ & {$[\mathrm{~A} 8]$} & 0.01 & $\begin{array}{l}\text { Grant et al. } \\
(1993 a, b)\end{array}$ \\
\hline$L_{\mathrm{h} j}$ & $\begin{array}{l}\text { ratio of nonlignin to lignin components in humified } \\
\text { hydrolysis products }\end{array}$ & & [A33] & $\begin{array}{l}0.10,0.05 \text {, and } \\
0.05 \text { for } j= \\
\text { protein, } \\
\text { carbohydrate, and } \\
\text { cellulose, } \\
\text { respectively }\end{array}$ & $\begin{array}{l}\text { Shulten and } \\
\text { Schnitzer (1997) }\end{array}$ \\
\hline$M$ & molecular mass of water & $\mathrm{g} \mathrm{mol}^{-1}$ & {$[\mathrm{~A} 15]$} & 18 & \\
\hline$M_{i, d, l, C}$ & heterotrophic microbial $\mathrm{C}$ used for decomposition & $\mathrm{g} \mathrm{C} \mathrm{m}^{-2}$ & {$[\mathrm{~A} 1, \mathrm{~A} 3 \mathrm{a}, \mathrm{A} 4]$} & & \\
\hline
\end{tabular}




\begin{tabular}{|c|c|c|c|c|}
\hline$M_{i, n, j, l, \mathrm{C}}$ & microbial C & $\mathrm{g} \mathrm{C} \mathrm{m}^{-2}$ & {$[\mathrm{~A} 13, \mathrm{~A} 17 \mathrm{~A} 23, \mathrm{~A} 2$} & \\
\hline & & & 5,A26, A30,A36] & \\
\hline$M_{i, n, j, l, \mathrm{~N}}$ & microbial $\mathrm{N}$ & $\mathrm{g} \mathrm{N} \mathrm{m}^{-2}$ & {$[\mathrm{~A} 18, \mathrm{~A} 27, \mathrm{~A} 29]$} & \\
\hline$M_{i, n, j, l, \mathrm{P}}$ & microbial P & $\mathrm{g} \mathrm{P} \mathrm{m}^{-2}$ & {$[\mathrm{~A} 24, \mathrm{~A} 29, \mathrm{~A} 26$} & \\
\hline & & & A36] & \\
\hline$M_{i, n, a, l, \mathrm{C}}$ & active microbial $\mathrm{C}$ from heterotrophic population $n$ associated & $\mathrm{g} \mathrm{C} \mathrm{m}^{-2}$ & {$[\mathrm{~A} 3, \mathrm{~A} 13, \mathrm{~A} 17$,} & \\
\hline & with $G_{i, l, \mathrm{C}}$ & & A30] & \\
\hline$\left[M_{i, n, a, l, \mathrm{C}}\right]$ & concentration of $M_{i, n, a}$ in soil water $=M_{i, n, a, l, \mathrm{C}} / \theta_{l}$ & $\mathrm{~g} \mathrm{C} \mathrm{m}^{-3}$ & {$[\mathrm{~A} 3, \mathrm{~A} 5]$} & \\
\hline$\left[\mathrm{NH}_{4}{ }_{i, n, j, l}\right]$ & concentration of $\mathrm{NH}_{4}{ }^{+}$at microbial surfaces & $\mathrm{g} \mathrm{N} \mathrm{m}^{-3}$ & [A26] & \\
\hline$\left[\mathbf{N H}_{4}{ }^{+} \mathrm{mn}\right]$ & concentration of $\mathrm{NH}_{4}{ }^{+}$at microbial surfaces below which & $\mathrm{g} \mathrm{N} \mathrm{m}^{-3}$ & [A26] & 0.0125 \\
\hline & $U_{\mathrm{NH}_{4}}=0$ & & & \\
\hline$\left[\mathrm{NO}_{3}^{-}{ }_{i, n, j, l}\right]$ & concentration of $\mathrm{NH}_{4}{ }^{+}$at microbial surfaces & $\mathrm{g} \mathrm{N} \mathrm{m}^{-3}$ & [A26] & \\
\hline$\left[\mathrm{NO}_{3}^{-} \mathrm{mn}\right]$ & concentration of $\mathrm{NO}_{3}{ }^{-}$at microbial surfaces below which & $\mathrm{g} \mathrm{N} \mathrm{m}^{-3}$ & [A26] & 0.03 \\
\hline & $U_{\mathrm{NO}_{3}}=0$ & & & \\
\hline$\left[\mathrm{H}_{2} \mathrm{PO}_{4 i, n, j, l}^{-}\right]$ & concentration of $\mathrm{H}_{2} \mathrm{PO}_{4}^{-}$at microbial surfaces & $\mathrm{g} \mathrm{N} \mathrm{m}^{-3}$ & [A26] & \\
\hline$\left[\mathrm{H}_{2} \mathrm{PO}_{4}^{-} \mathrm{mn}\right]$ & concentration of $\mathrm{H}_{2} \mathrm{PO}_{4}{ }^{-}$at microbial surfaces below which & $\mathrm{g} \mathrm{N} \mathrm{m}^{-3}$ & [A26] & 0.002 \\
\hline
\end{tabular}

$$
U_{\mathrm{PO}_{4}}=0
$$




\begin{tabular}{|c|c|c|c|c|}
\hline$\left[\mathrm{O}_{2 \mathrm{~m} i, n, l}\right]$ & $\mathrm{O}_{2}$ concentration at heterotrophic microsites & $\mathrm{g} \mathrm{O}_{2} \mathrm{~m}^{-3}$ & [A17] & \\
\hline$\left[\mathrm{O}_{2 s l}\right]$ & $\mathrm{O}_{2}$ concentration in soil solution & $\mathrm{g} \mathrm{O}_{2} \mathrm{~m}^{-3}$ & [A17] & \\
\hline$Q_{i, l, \mathrm{C}}$ & $D O C$ from products of $D_{S i, j, l, \mathrm{C}}[\mathrm{A} 3]$ and $\left.D_{Z i, j, l, \mathrm{C}}\right)[\mathrm{A} 5]$ & $\mathrm{g} \mathrm{C} \mathrm{m}^{-2}$ & {$[\mathrm{~A} 8, \mathrm{~A} 13, \mathrm{~A} 22]$} & \\
\hline$\left[Q_{i, l, \mathrm{C}}\right]$ & solution concentration of $Q_{i, l, \mathrm{C}}$ & $\mathrm{g} \mathrm{C} \mathrm{Mg}^{-1}$ & {$[\mathrm{~A} 8, \mathrm{~A} 13]$} & \\
\hline$Q_{i, l, \mathrm{~N}, \mathrm{P}}$ & $D O N$ and DOP from products of $\left(D_{S i, j, l, \mathrm{~N}, \mathrm{P}}+D_{Z i, j, l, \mathrm{~N}, \mathrm{P}}\right)$ & $\mathrm{g} \mathrm{N}$ or $\mathrm{P} \mathrm{m}^{-2}$ & {$[\mathrm{~A} 9, \mathrm{~A} 22]$} & \\
\hline$q_{m}$ & constant for reallocating $M_{i, a, l, \mathrm{C}}$ to $M_{i, d, l, \mathrm{C}}$ & - & [A3a] & 0.5 \\
\hline $\boldsymbol{R}$ & gas constant & 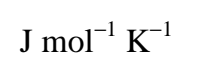 & {$[\mathrm{A} 6, \mathrm{~A} 15, \mathrm{C} 10]$} & 8.3143 \\
\hline$R_{\Phi i, n=f, j, l}$ & $\begin{array}{l}\text { respiration for non-symbiotic } \mathrm{N}_{2} \text { fixation by heterotrophic } \\
\text { diazotrophs }(n=f)\end{array}$ & $\mathrm{gC} \mathrm{m}^{-2} \mathrm{~h}^{-1}$ & [A28] & \\
\hline$R_{g i, n, l}$ & $\begin{array}{l}\text { growth respiration of } M_{i, n, a, l} \text { on } Q_{i, l, \mathrm{C}} \text { under nonlimiting } \mathrm{O}_{2} \text { and } \\
\text { nutrients }\end{array}$ & $\mathrm{gCg} \mathrm{C}^{-1} \mathrm{~h}^{-1}$ & [A20] & \\
\hline$R_{\mathrm{h}}$ & $\begin{array}{l}\text { total heterotrophic respiration of all } M_{i, n, a, l} \text { under ambient } \\
\text { DOC, } \mathrm{O}_{2} \text {, nutrients, } \theta \text { and temperature }\end{array}$ & $\mathrm{g} \mathrm{C} \mathrm{m}^{-2} \mathrm{~h}^{-1}$ & [A11] & \\
\hline$R_{\mathrm{hin,l,l}}$ & $\begin{array}{l}\text { heterotrophic respiration of } M_{i, n, a, l} \text { under ambient DOC, } \mathrm{O}_{2} \text {, } \\
\text { nutrients, } \theta \text { and temperature }\end{array}$ & $\mathrm{g} \mathrm{C} \mathrm{m}^{-2} \mathrm{~h}^{-1}$ & $\begin{array}{l}{[\mathrm{A} 5, \mathrm{~A} 11, \mathrm{~A} 14, \mathrm{~A} 2} \\
0, \mathrm{~A} 21, \mathrm{~A} 25]\end{array}$ & \\
\hline $\boldsymbol{R}_{\mathrm{hi}, n, l}$ & $\begin{array}{l}\text { specific heterotrophic respiration of } M_{i, n, a, l} \text { under nonlimiting } \\
\mathrm{O}_{2} \text {, DOC, } \theta \text { and } 25^{\circ} \mathrm{C}\end{array}$ & $\mathrm{g} \mathrm{Cg} \mathrm{C}^{-1} \mathrm{~h}^{-1}$ & {$[\mathrm{~A} 12, \mathrm{~A} 13]$} & \\
\hline
\end{tabular}




\begin{tabular}{|c|c|c|c|c|c|}
\hline $\boldsymbol{R}_{\mathrm{h} n}{ }^{\prime}$ & $\begin{array}{l}\text { specific heterotrophic respiration of } M_{i, n, a, l} \text { under nonlimiting } \\
\text { DOC, } \mathrm{O}_{2} \text {, nutrients, } \theta \text { and } 25^{\circ} \mathrm{C}\end{array}$ & $\mathrm{g} \mathrm{C} \mathrm{g} \mathrm{C}^{-1} \mathrm{~h}^{-1}$ & [A12] & 0.125 & $\begin{array}{l}\text { Shields et al. } \\
\text { (1973) }\end{array}$ \\
\hline$R_{\mathrm{h} i, n, l}{ }^{\prime}$ & $\begin{array}{l}\text { heterotrophic respiration of } M_{i, n, a, l} \text { under nonlimiting } \mathrm{O}_{2} \text { and } \\
\text { ambient DOC, nutrients, } \theta \text { and temperature }\end{array}$ & $\mathrm{gC} \mathrm{m}^{-2} \mathrm{~h}^{-1}$ & {$[\mathrm{~A} 13, \mathrm{~A} 14, \mathrm{~A} 16]$} & & \\
\hline $\boldsymbol{R}_{\mathrm{m}}$ & specific maintenance respiration at $25^{\circ} \mathrm{C}$ & $\mathrm{g} \mathrm{C} \mathrm{g} \mathrm{N}^{-1} \mathrm{~h}^{-1}$ & [A18] & 0.0115 & $\begin{array}{l}\text { Barnes et al. } \\
\text { (1998) }\end{array}$ \\
\hline$R_{\mathrm{m}, n, j, l}$ & maintenance respiration by $M_{i, n, j, l}$ & $\mathrm{~g} \mathrm{C} \mathrm{m}^{-2} \mathrm{~h}^{-1}$ & $\begin{array}{l}{[\mathrm{A} 18, \mathrm{~A} 20, \mathrm{~A} 21, \mathrm{~A}} \\
25]\end{array}$ & & \\
\hline$r_{\mathrm{w} l}$ & radius of $r_{\mathrm{m}}+$ water film at current water content & $\mathrm{m}$ & [A17] & & \\
\hline$r_{\mathrm{m}}$ & radius of heterotrophic microsite & $\mathrm{m}$ & [A17] & $2.5 \times 10^{-6}$ & \\
\hline$r_{\mathrm{w} l}$ & thickness of water films & $\mathrm{m}$ & [A17] & & \\
\hline$S$ & change in entropy & $\mathrm{J} \mathrm{mol}^{-1} \mathrm{~K}^{-1}$ & {$[\mathrm{~A} 6, \mathrm{C} 10]$} & 710 & $\begin{array}{l}\text { Sharpe and } \\
\text { DeMichelle } \\
\text { (1977) }\end{array}$ \\
\hline$\left[S_{i, j, l, \mathrm{C}}\right]$ & concentration of $S_{i, j, l, \mathrm{C}}$ in soil & $\mathrm{g} \mathrm{C} \mathrm{Mg}^{-1}$ & {$[\mathrm{~A} 4 \mathrm{a}]$} & & \\
\hline$S_{i, j, l, \mathrm{C}}$ & mass of colonized litter, POC or humus C & $\mathrm{g} \mathrm{C} \mathrm{m}^{-2}$ & $\begin{array}{l}{[\mathrm{A} 2 \mathrm{a}, \mathrm{A} 5, \mathrm{~A} 7 \mathrm{a}, \mathrm{A} 3} \\
\text { 3] }\end{array}$ & & \\
\hline$S_{i, j, l, C}^{\prime}$ & mass of uncolonized litter, $\mathrm{POC}$ or humus $\mathrm{C}$ & $\mathrm{g} \mathrm{C} \mathrm{m}^{-2}$ & [A5] & & \\
\hline
\end{tabular}




\begin{tabular}{|c|c|c|c|c|}
\hline$S_{i, j, l, \mathrm{~N}, \mathrm{P}}$ & mass of litter, $\mathrm{POC}$ or humus $\mathrm{N}$ or $\mathrm{P}$ & $\mathrm{g} \mathrm{N}$ or $\mathrm{P} \mathrm{m}^{-2}$ & {$[\mathrm{~A} 7 \mathrm{a}, \mathrm{A} 33]$} & \\
\hline$T_{s l}$ & soil temperature & K & {$[\mathrm{A} 6, \mathrm{~A} 15 . \mathrm{A} 19]$} & \\
\hline$U_{i, n, l \mathrm{C}}$ & uptake of $Q_{i, l, \mathrm{C}}$ by $\Sigma_{n} M_{i, n, a, l}$ under limiting nutrient availability & $\mathrm{g} \mathrm{C} \mathrm{m}^{-2} \mathrm{~h}^{-1}$ & {$[\mathrm{~A} 5, \mathrm{~A} 21, \mathrm{~A} 22, \mathrm{~A} 2$} & \\
\hline & & & 5] & \\
\hline$U_{i, n, \mathrm{~N}, \mathrm{P}}$ & $\begin{array}{l}\text { uptake of } Q_{i, l, \mathrm{~N}, \mathrm{P}} \text { by } \Sigma_{n} M_{i, n, a, l} \text { under limiting nutrient } \\
\text { availability }\end{array}$ & $\mathrm{g} \mathrm{N}$ or $\mathrm{P} \mathrm{m}^{-2} \mathrm{~h}^{-1}$ & {$[\mathrm{~A} 22, \mathrm{~A} 29]$} & \\
\hline$U_{\mathrm{NH} 4 i, n, j, l}$ & $\mathrm{NH}_{4}{ }^{+}$uptake by microbes & $\mathrm{g} \mathrm{N} \mathrm{m}^{-2} \mathrm{~h}^{-1}$ & {$[\mathrm{~A} 26, \mathrm{~A} 27, \mathrm{~A} 29]$} & \\
\hline$U_{\mathrm{NH}_{4}}^{\prime}$ & maximum $U_{\mathrm{NH}_{4}}$ at $25^{\circ} \mathrm{C}$ and non-limiting $\mathrm{NH}_{4}{ }^{+}$ & $\mathrm{g} \mathrm{N} \mathrm{m}^{-2} \mathrm{~h}^{-1}$ & [A26] & $5.0 \times 10^{-3}$ \\
\hline$U_{\mathrm{NO} O 3 i, n, j, l}$ & $\mathrm{NO}_{3}{ }^{-}$uptake by microbes & $\mathrm{g} \mathrm{N} \mathrm{m}^{-2} \mathrm{~h}^{-1}$ & {$[\mathrm{~A} 26, \mathrm{~A} 27, \mathrm{~A} 29]$} & \\
\hline$U_{\mathrm{NO}_{3}}^{\prime}$ & maximum $U_{\mathrm{NO}_{3}}$ at $25^{\circ} \mathrm{C}$ and non-limiting $\mathrm{NO}_{3}^{-}$ & $\mathrm{g} \mathrm{N} \mathrm{m}^{-2} \mathrm{~h}^{-1}$ & [A26] & $5.0 \times 10^{-3}$ \\
\hline$U_{\mathrm{O} 2 i, n}$ & $\mathrm{O}_{2}$ uptake by $M_{i, n, a, l}$ under ambient $\mathrm{O}_{2}$ & $\mathrm{~g} \mathrm{~m}^{-2} \mathrm{~h}^{-1}$ & {$[\mathrm{~A} 14, \mathrm{~A} 17]$} & \\
\hline$U^{\prime} \mathrm{O} 2, n$ & $\mathrm{O}_{2}$ uptake by $M_{i, n, a, l}$ under nonlimiting $\mathrm{O}_{2}$ & $\mathrm{~g} \mathrm{~m}^{-2} \mathrm{~h}^{-1}$ & {$[\mathrm{~A} 14, \mathrm{~A} 16, \mathrm{~A} 17]$} & \\
\hline$U_{\mathrm{PO} 4 i, n, j, l}$ & $\mathrm{H}_{2} \mathrm{PO}_{4}^{-}$uptake by microbes & $\mathrm{g} \mathrm{N} \mathrm{m}^{-2} \mathrm{~h}^{-1}$ & {$[\mathrm{~A} 26, \mathrm{~A} 27, \mathrm{~A} 29]$} & \\
\hline$U^{\prime} \mathbf{P O}_{4}$ & maximum $U_{\mathrm{PO}_{4}}$ at $25^{\circ} \mathrm{C}$ and non-limiting $\mathrm{H}_{2} \mathrm{PO}_{4}^{-}$ & $\mathrm{g} \mathrm{N} \mathrm{m}^{-2} \mathrm{~h}^{-1}$ & [A26] & $5.0 \times 10^{-3}$ \\
\hline$X_{i, l, \mathrm{C}}$ & adsorbed $\mathrm{C}$ hydrolysis products & $\mathrm{g} \mathrm{C} \mathrm{Mg}^{-1}$ & {$[\mathrm{~A} 8, \mathrm{~A} 10]$} & \\
\hline$X_{i, l, \mathrm{~N}, \mathrm{P}}$ & adsorbed $\mathrm{N}$ or $\mathrm{P}$ hydrolysis products & $\mathrm{g} \mathrm{P} \mathrm{Mg}^{-1}$ & [A10] & \\
\hline$y$ & selected to give a $Q_{10}$ for $f_{\mathrm{tm}}$ of 2.25 & & [A19] & 0.081 \\
\hline
\end{tabular}




\section{Appendix B: Soil-plant water relations}

\section{Canopy transpiration}

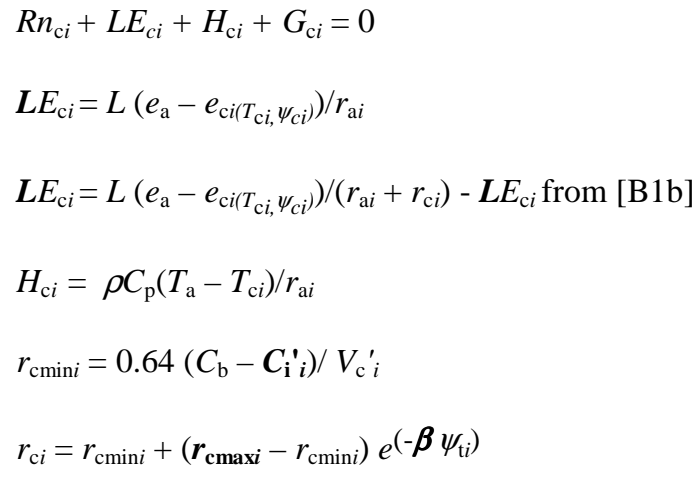

$r_{\mathrm{c}}$ constrained by water status 
$r_{\mathrm{a} i}=\left\{\left(\ln \left(\left(z_{\mathrm{u}}-z_{\mathrm{di}}\right) / z_{\mathrm{ri}}\right)^{2} /\left(\boldsymbol{K}^{2} u_{\mathrm{a}}\right)\right\} /(1-10 R i)\right.$

$R i=\left\{g\left(z_{\mathrm{u}}-z_{\mathrm{ri}}\right) /\left(u_{\mathrm{a}}^{2} T_{\mathrm{a}}\right)\right\}\left(T_{\mathrm{a}}-T_{\mathrm{c}}\right)$

$\psi_{\mathrm{t} i}=\psi_{\mathrm{c} i}-\psi_{\pi}$

\section{Root and mycorrhizal water uptake}

$U_{\mathrm{w} i}=\Sigma_{l} \Sigma_{\mathrm{r}} U_{\mathrm{w} i, r, l}$

$U_{\mathrm{w} i, r, l}=\left(\psi_{\mathrm{c} i}{ }^{\prime}-\psi_{\mathrm{s}}{ }^{\prime}\right) /\left(\Omega_{\mathrm{s} i, r, l}+\Omega_{\mathrm{r} i, r, l}+\Sigma_{x} \Omega_{\mathrm{a} i, r, l, x}\right)$

$\psi_{\mathrm{c} i}^{\prime}=\psi_{\mathrm{c} i}+0.01 z_{\mathrm{b} i}$

$\psi_{\mathrm{s}}{ }^{\prime}=\psi_{\mathrm{s} l}-0.01 z_{l}$

$\Omega_{\mathrm{si}, r, l}=\ln \left\{\left(d_{i, r, l} / r_{i, r, l}\right) /\left(2 \pi L_{i, r, l} \kappa_{\mathrm{ri}, r, l}\right)\right\} \theta_{\mathrm{wl}} / \theta_{\mathrm{p} l}$

$\Omega_{\mathrm{ri}, r, l}=\boldsymbol{\Omega}_{\mathrm{ri}, r} / L_{i, r, l}$

$\boldsymbol{\Omega}_{\mathrm{a}, r, l, x=1}=\boldsymbol{\Omega}_{\mathrm{a} i, r} z_{l} /\left\{n_{i, r, l, l}\left(r_{i, r, l, l} / \boldsymbol{r}_{i, r}^{\prime}\right)^{4}\right\}+\boldsymbol{\gamma} \boldsymbol{\Omega}_{\mathrm{a} i, r} z_{\mathrm{b} i} /\left\{n_{i, r, l, l}\left(r_{\mathrm{b} i} / r_{\mathrm{b} i}\right)^{4}\right\} \Sigma_{i, r, l}\left(M_{i, r, l}\right) / M_{i, r, l}$

$\Omega_{\mathrm{a} i, r, l, x=2}=\boldsymbol{\Omega}_{\mathrm{a} i, r}\left(L_{i, r, l, 2} / n_{i, r, l, 2}\right) /\left\{n_{i, r, l, 2}\left(r_{i, r, l, 2} / r_{i, r}^{\prime}\right)^{4}\right\}$

$\delta L_{i, r, l, 1} / \delta t=\delta M_{i, r, l, 1} / \delta t v_{r} /\left\{\rho_{r}\left(1-\theta_{\mathrm{P}_{i, r}}\right)\left(\pi r_{i, r, l, 1}^{2}\right)\right\}$ $r_{\mathrm{a}}$ driven by windspeed, surface

$r_{\mathrm{a}}$ adjusted for stability $v s$.

buoyancy

[B4]

[B5]

$U_{\text {w }}$ along hydraulic gradient

[B6]

[B7]

[B8]

[B9]

[B10]

[B11]

[B12]

[B13]

\section{Canopy water potential}


[B1-B4] (LHS) equals uptake from

[B5-B13] + change in storage

(RHS)

\section{Definition of variables in appendix B}

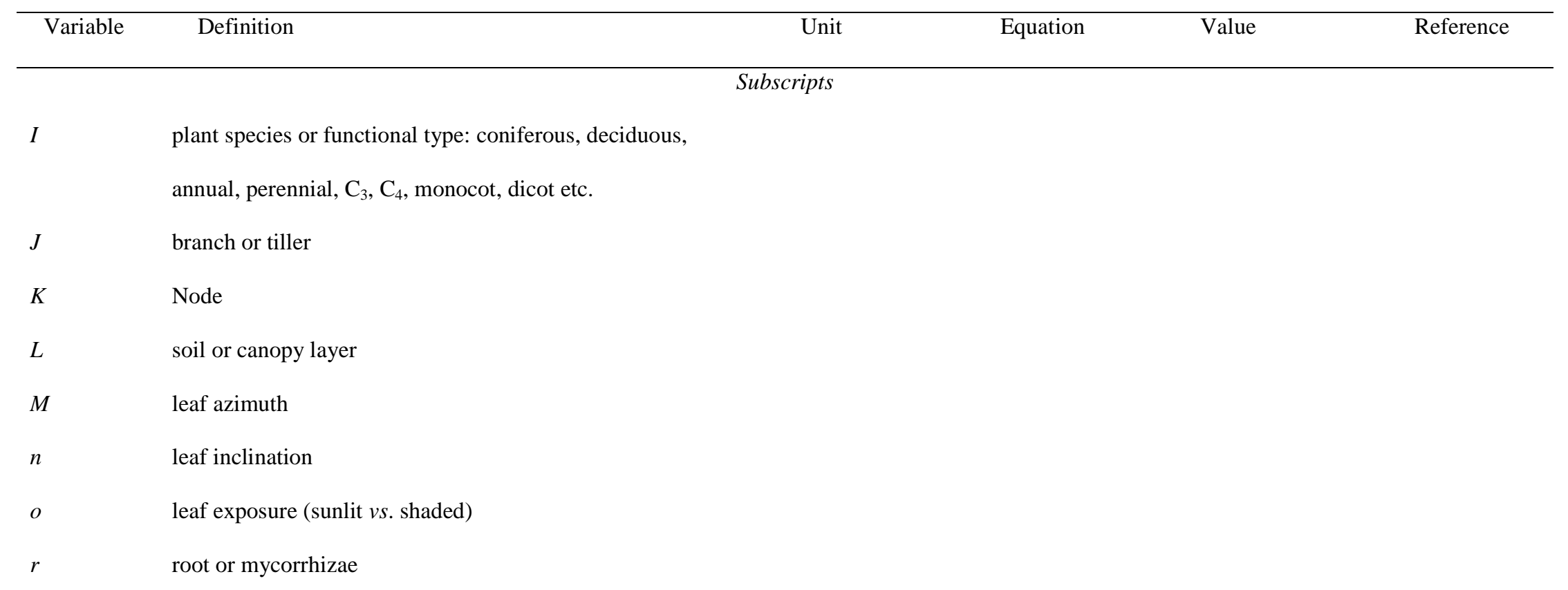




\begin{tabular}{|c|c|c|c|c|c|}
\hline$\beta$ & stomatal resistance shape parameter & $\mathrm{MPa}^{-1}$ & {$[\mathrm{~B} 2 \mathrm{~b}, \mathrm{C} 4, \mathrm{C} 9]$} & -5.0 & $\begin{array}{l}\text { Grant and } \\
\text { Flanagan (2007) }\end{array}$ \\
\hline$C_{\mathrm{b}}$ & {$\left[\mathrm{CO}_{2}\right]$ in canopy air } & $\mu \mathrm{mol} \mathrm{mol}{ }^{-1}$ & {$[\mathrm{~B} 2, \mathrm{C} 2, \mathrm{C} 5]$} & & \\
\hline$C_{\mathrm{i}^{\prime} i}$ & {$\left[\mathrm{CO}_{2}\right]$ in canopy leaves at $\psi_{\mathrm{c}_{i}}=0 \mathrm{MPa}$} & $\mu \mathrm{mol} \mathrm{mol}{ }^{-1}$ & {$[\mathrm{~B} 2]$} & $0.70 C_{\mathrm{b}}$ & Larcher (2001) \\
\hline$d_{i, r, l}$ & half distance between adjacent roots & $\mathrm{m}$ & [B9] & & \\
\hline$E_{\mathrm{ci}}$ & canopy transpiration & $\mathrm{m}^{3} \mathrm{~m}^{-2} \mathrm{~h}^{-1}$ & {$[\mathrm{~B} 1, \mathrm{~B} 14]$} & & \\
\hline$e_{\mathrm{a}}$ & atmospheric vapor density at $T_{\mathrm{a}}$ and ambient humidity & $\mathrm{g} \mathrm{m}^{-3}$ & {$[\mathrm{~B} 1]$} & & \\
\hline$e_{\mathrm{ci}\left(T_{\mathrm{c} i}, \psi_{c i}\right)}$ & canopy vapor density at $T_{\mathrm{c}_{i}}$ and $\psi_{\mathrm{c}_{i}}$ & $\mathrm{~g} \mathrm{~m}^{-3}$ & {$[\mathrm{~B} 1]$} & & \\
\hline$G_{\mathbf{c} i}$ & canopy storage heat flux & $\mathrm{W} \mathrm{m} \mathrm{m}^{-2}$ & {$[\mathrm{~B} 1]$} & & \\
\hline$H_{\mathbf{c} i}$ & canopy sensible heat flux & $\mathrm{W} \mathrm{m} \mathrm{m}^{-2}$ & [B1] & & \\
\hline K & von Karman's constant & & [B3a] & 0.41 & \\
\hline$\kappa_{\mathrm{r}, i, l}$ & hydraulic conductivity between soil and root surface & $\mathrm{m}^{2} \mathrm{MPa}^{-1} \mathrm{~h}^{-1}$ & [B9] & & \\
\hline$\gamma$ & $\begin{array}{l}\text { scaling factor for bole axial resistance from primary root axial } \\
\text { resistance }\end{array}$ & - & {$[\mathrm{B} 11]$} & $1.6 \times 10^{4}$ & Grant et al. (2007) \\
\hline$L$ & latent heat of evaporation & $\mathrm{Jg}^{-1}$ & [B1] & 2460 & \\
\hline$L E_{c i}$ & latent heat flux between canopy and atmosphere & $\mathrm{W} \mathrm{m}^{-2}$ & {$[\mathrm{~B} 1]$} & & \\
\hline
\end{tabular}




\begin{tabular}{|c|c|c|c|c|c|}
\hline$L_{i, r, l}$ & length of roots or mycorrhizae & $\mathrm{m} \mathrm{m}^{-2}$ & {$[\mathrm{~B} 9, \mathrm{~B} 10, \mathrm{~B} 12, \mathrm{~B} 13$} & & \\
\hline & & & ] & & \\
\hline$M_{i, r, l}$ & mass of roots or mycorrhizae & $\mathrm{g} \mathrm{m}^{-2}$ & {$[\mathrm{~B} 11, \mathrm{~B} 13]$} & & \\
\hline$n_{i, r, l, x}$ & number of primary $(x=1)$ or secondary $(x=2)$ axes & $\mathrm{m}^{-2}$ & {$[\mathrm{~B} 11, \mathrm{~B} 12]$} & & \\
\hline $\boldsymbol{\Omega}_{\mathrm{a}, \boldsymbol{r}}$ & $\begin{array}{l}\text { axial resistivity to water transport along root or mycorrhizal } \\
\text { axes }\end{array}$ & $\mathrm{MPa} \mathrm{h} \mathrm{m}{ }^{-4}$ & {$[\mathrm{~B} 11, \mathrm{~B} 12]$} & $\begin{array}{l}4.0 \times 10^{9} \\
\text { deciduous } \\
1.0 \times 10^{10} \\
\text { coniferous }\end{array}$ & Larcher (2001) \\
\hline$\Omega_{\mathrm{a} i, r, l, x}$ & $\begin{array}{l}\text { axial resistance to water transport along axes of primary ( } x= \\
\text { 1) or secondary }(x=2) \text { roots or mycorrhizae }\end{array}$ & $\mathrm{MPa} \mathrm{h} \mathrm{m}{ }^{-1}$ & {$[\mathrm{~B} 6, \mathrm{~B} 11, \mathrm{~B} 12]$} & & \\
\hline $\boldsymbol{\Omega}_{\mathrm{r} i, r}$ & $\begin{array}{l}\text { radial resistivity to water transport from surface to axis of } \\
\text { roots or mycorrhizae }\end{array}$ & $\mathrm{MPa} \mathrm{h} \mathrm{m} \mathrm{m}^{-2}$ & [B10] & $1.0 \times 10^{4}$ & $\begin{array}{l}\text { Doussan et al. } \\
\text { (1998) }\end{array}$ \\
\hline$\Omega_{\mathrm{r}, r, l}$ & $\begin{array}{l}\text { radial resistance to water transport from surface to axis of } \\
\text { roots or mycorrhizae }\end{array}$ & $\mathrm{MPa} \mathrm{h} \mathrm{m}{ }^{-1}$ & {$[\mathrm{~B} 6, \mathrm{~B} 10]$} & & \\
\hline$\Omega_{\mathrm{si}, r, l}$ & $\begin{array}{l}\text { radial resistance to water transport from soil to surface of } \\
\text { roots or mycorrhizae }\end{array}$ & MPa h m ${ }^{-1}$ & {$[\mathrm{~B} 6, \mathrm{~B} 9]$} & & \\
\hline$\theta_{\mathrm{w} l}$ & soil water content & $\mathrm{m}^{3} \mathrm{~m}^{-3}$ & [B9] & & \\
\hline$\theta_{\mathrm{p} l}$ & soil porosity & $\mathrm{m}^{3} \mathrm{~m}^{-3}$ & [B9] & & \\
\hline
\end{tabular}




\begin{tabular}{|c|c|c|c|c|c|}
\hline$\theta_{\mathrm{P}_{i, r}}$ & root porosity & $\mathrm{m}^{3} \mathrm{~m}^{-3}$ & {$[\mathrm{~B} 13]$} & & \\
\hline \multirow[t]{2}{*}{$R i$} & \multicolumn{2}{|l|}{ Richarson number } & \multicolumn{2}{|l|}{$[\mathrm{B} 3 \mathrm{a}, \mathrm{B} 3 \mathrm{~b}]$} & van Bavel and \\
\hline & & & & & Hillel (1976) \\
\hline$R n_{\mathrm{c} i}$ & canopy net radiation & $\mathrm{W} \mathrm{m}^{-2}$ & \multicolumn{2}{|l|}{$[\mathrm{B} 1]$} & \\
\hline$r_{\mathrm{a} i}$ & aerodynamic resistance to vapor flux from canopy & $\mathrm{s} \mathrm{m}^{-1}$ & \multicolumn{2}{|l|}{$[\mathrm{B} 1, \mathrm{~B} 3 \mathrm{a}]$} & \\
\hline$r_{\mathrm{b} i}$ & radius of bole at ambient $\psi_{c_{i}}$ & $\mathrm{~m}$ & \multicolumn{2}{|l|}{ [B11] } & \\
\hline$r_{\mathrm{b}^{\prime} i}$ & radius of bole at $\psi_{\mathrm{c}_{i}}=0 \mathrm{MPa}$ & $\mathrm{m}$ & \multicolumn{2}{|l|}{ [B11] } & \\
\hline$r_{\mathrm{c} i}$ & canopy stomatal resistance to vapor flux & $\mathrm{s} \mathrm{m}^{-1}$ & \multicolumn{2}{|l|}{$[\mathrm{B} 1, \mathrm{~B} 2 \mathrm{~b}]$} & \\
\hline$r_{\text {cmaxi }}$ & canopy cuticular resistance to vapor flux & $\mathrm{s} \mathrm{m}^{-1}$ & {$[\mathrm{~B} 2 \mathrm{~b}]$} & $5.0 \times 10^{3}$ & Larcher (2001) \\
\hline$r_{\mathrm{cmin} i}$ & minimum $\mathrm{r}_{\mathrm{c}_{i}}$ at $\psi_{\mathrm{c}_{i}}=0 \mathrm{MPa}$ & $\mathrm{s} \mathrm{m}^{-1}$ & {$[\mathrm{~B} 2, \mathrm{~B} 2 \mathrm{~b}]$} & & \\
\hline \multirow[t]{2}{*}{$r_{i, r l, l, x}$} & radius of primary $(x=1)$ or secondary $(x=2)$ roots or & $\mathrm{m}$ & \multicolumn{2}{|l|}{$[\mathrm{B} 9, \mathrm{~B} 11, \mathrm{~B} 12, \mathrm{~B} 13$} & \\
\hline & mycorrhizae at ambient $\psi_{\mathrm{r}_{i l, z}}$ & & ] & & \\
\hline \multirow[t]{4}{*}{$\boldsymbol{r}_{i, r}^{\prime}$} & \multirow[t]{4}{*}{ radius of secondary roots or mycorrhizae at $\psi_{\mathrm{r}_{i} l, z}=0 \mathrm{MPa}$} & \multirow[t]{4}{*}{$\mathrm{m}$} & \multirow[t]{4}{*}[\mathrm{B}11,\mathrm{B}12]{} & $2.0 \times 10^{-4}$ tree & \\
\hline & & & & $1.0 \times 10^{-4}$ bush & \\
\hline & & & & $0.05 \times 10^{-4}$ & \\
\hline & & & & mycorrhizae & \\
\hline$\rho_{r}$ & root specific density & 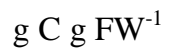 & [B13] & 0.05 & Grant (1998a) \\
\hline
\end{tabular}




\begin{tabular}{|c|c|c|c|c|c|}
\hline$T_{\mathrm{a}}$ & air temperature & $\mathrm{K}$ & [B3b] & & \\
\hline$T_{\mathrm{c}}$ & canopy temperature & $\mathrm{K}$ & [B3b] & & \\
\hline$U_{\mathrm{w} i}$ & total water uptake from all rooted soil layers & $\mathrm{m}^{3} \mathrm{~m}^{-2} \mathrm{~h}^{-1}$ & {$[\mathrm{~B} 5, \mathrm{~B} 14]$} & & \\
\hline$U_{\mathrm{w}, i, l}$ & $\begin{array}{l}\text { water uptake by root and mycorrhizal surfaces in each soil } \\
\text { layer }\end{array}$ & $\mathrm{m}^{3} \mathrm{~m}^{-2} \mathrm{~h}^{-1}$ & {$[\mathrm{~B} 5, \mathrm{~B} 6]$} & & \\
\hline$u_{\mathrm{a}}$ & wind speed measured at $z_{\mathrm{u}}$ & $\mathrm{m} \mathrm{s}^{-1}$ & {$[\mathrm{~B} 3 \mathrm{a}, \mathrm{B} 3 \mathrm{~b}]$} & & \\
\hline$V_{\mathrm{c}}{ }^{\prime}$ & potential canopy $\mathrm{CO}_{2}$ fixation rate at $\psi_{\mathrm{c}_{i}}=0 \mathrm{MPa}$ & $\mu \mathrm{mol} \mathrm{m} \mathrm{m}^{-2}$ & [B2] & & \\
\hline$v_{r}$ & root specific volume & $\mathrm{m}^{3} \mathrm{~g} \mathrm{FW}^{-1}$ & [B13] & $10^{-6}$ & Grant (1998a) \\
\hline$X_{\mathrm{c} i}$ & canopy capacitance & $\mathrm{m}^{3} \mathrm{~m}^{-2} \mathrm{MPa}^{-1}$ & [B14] & & \\
\hline$\psi_{\mathrm{c} i}$ & canopy water potential & MPa & {$[\mathrm{B} 4, \mathrm{~B} 7, \mathrm{~B} 14]$} & & \\
\hline$\psi_{\mathrm{c}^{\prime} i}^{\prime}$ & $\psi_{c i}+$ canopy gravitational potential & MPa & {$[\mathrm{B} 6, \mathrm{~B} 7]$} & & \\
\hline$\psi_{\pi i}$ & canopy osmotic potential & MPa & [B4] & & \\
\hline$\psi_{\mathrm{s} l}$ & soil water potential & MPa & [B8] & & \\
\hline$\psi_{\mathrm{s}}^{\prime} l$ & $\psi_{s l}+$ soil gravitational potential & $\mathrm{MPa}$ & {$[\mathrm{B} 6, \mathrm{~B} 8]$} & & \\
\hline$\psi_{\mathrm{ti}}$ & canopy turgor potential & $\mathrm{MPa}$ & {$[\mathrm{B} 2 \mathrm{~b}, \mathrm{~B} 4]$} & 1.25 at $\psi_{\mathrm{c}}=0$ & \\
\hline$z_{\mathrm{b} i}$ & length of bole from soil surface to top of canopy & $\mathrm{m}$ & {$[\mathrm{B} 7, \mathrm{~B} 11]$} & & \\
\hline$z_{\mathrm{d} i}$ & canopy zero-plane displacement height & $\mathrm{m}$ & [B3a] & & Perrier (1982) \\
\hline $\mathrm{z}_{l}$ & depth of soil layer below surface & $\mathrm{m}$ & {$[\mathrm{B} 8, \mathrm{~B} 11]$} & & \\
\hline
\end{tabular}


[B3a,B3b]

\section{Appendix C: Gross primary productivity and autotrophic respiration}

\section{$\mathrm{C}_{3}$ gross primary productivity}

$G P P=\sum_{i, j, k, l, m, n, o}\left(V_{\mathrm{c} i, j, k, l, m, n, o}=V_{\mathrm{g} i, j, k, l, m, n, o}\right) A_{i, j, k, l, m, n, o}$

solve for $C_{\mathrm{i} i, j, k, l, m, n, o}$ at which

$V_{\mathrm{gi}, j, k, l, m, n, o}=\left(C_{\mathrm{b}}-C_{\mathrm{i} i, j, k, l, m, n, o}\right) / r_{1 i, j, k, l, m, n, o}$

$V_{\mathrm{c} i, j, k, l, m, n, o}=\min \left\{V_{\mathrm{b} i, j, k, l, m, n, o}, V_{\mathrm{j} i, j, k, l, m, n, o}\right\}$

$V_{\mathrm{c} i, j, k, l, m, n, o}=V_{\mathrm{g} i, j, k, l, m, n, \mathrm{o}}$

diffusion

$r_{l i, j, k, l, m, n, o}=r_{\operatorname{lmin} i, j, k, l, m, n, o}+\left(r_{\operatorname{lmax} i}-r_{\operatorname{lmin} i, j, k, l, m, n, o}\right) e^{\left(-\beta \psi_{\mathrm{ti}}\right)}$

carboxylation

[C3]

$r_{1}$ is leaf-level equivalent of $r_{\mathrm{c}}$

$r_{\mathrm{lmin} i, j, k, l, m, n, o}=\left(C_{\mathrm{b}}-C_{\mathbf{i}}{ }^{\prime}{ }_{i}\right) / V_{\mathrm{c}}{ }^{\prime},{ }_{j, k, k, l, m, n, o}$

minimum $r_{1}$ is driven by 
$\left.V_{\mathrm{b} i, j, k, l, m, n, o}=V_{\mathrm{bmax} i, j, k}\left(C_{\mathrm{c} i, j, k, l, m, n, o}-\Gamma_{i, j, k}\right) /\left(C_{\mathrm{c} i, j, k, l, m, n, o}\right)+K_{\mathrm{c}_{i}}\right) f_{\psi i, j, k, l, m, n, o} f_{\mathrm{iC} i}$

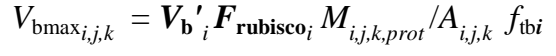

$\Gamma_{i, j, k}=0.5 O_{\mathrm{c}} V_{\operatorname{omax}_{i, j, k}} \boldsymbol{K}_{\mathbf{c}_{i}} /\left(V_{\operatorname{bmax}_{i, j, k}} \boldsymbol{K}_{\mathbf{o}_{i}}\right)$

$V_{\text {omax }_{i, j, k}}=\boldsymbol{V}_{\mathbf{o}_{i}}{ }_{i} \boldsymbol{F}_{\text {rubisco }_{i}} M_{i, j, k, p r o t} / A_{i, j, k} f_{\text {toi }}$

$K_{\mathrm{c}_{i}}=\boldsymbol{K}_{\mathbf{c}_{i}} f_{\mathrm{tkci}}\left(1+O_{\mathrm{c}} /\left(\boldsymbol{K}_{\mathbf{o}_{i}} f_{\mathrm{tko} i}\right)\right)$

$V_{\mathrm{j} i, j, k, l, m, n, o}=J_{i, j, k, l, m, n, o} Y_{i, j, k, l, m, n, o} f_{\psi i, j, k, l, m, n, o} f_{\mathrm{iC} i}$

$J_{i, j, k, l, m, n, o}=\left(\boldsymbol{\varepsilon} I_{i, l, m, n, o}+J_{\max i, j, k}-\left(\left(\varepsilon I_{i, l, m, n, o}+J_{\max i, j, k}\right)^{2}-4 \alpha \boldsymbol{\varepsilon} I_{i, l, m, n, o} J_{\max i, j, k}\right)^{0.5}\right) /(2 \alpha)$

$J_{\text {maxi,j,k }}=\boldsymbol{V}_{\mathbf{j}_{i}{ }_{i}} \boldsymbol{F}_{\text {chlorophyll }} M_{i, j, k, p r o t} / A_{i, j, k} f_{\mathrm{tj} i}$

$f_{\psi i, j, k, l, m, n, o}=\left(r_{\operatorname{lmin}_{i, j, k, l, m, n, o}} / r_{\mathrm{l}_{i, j, k, l, m, n, o}}\right)^{0.5}$
$\mathrm{CO}_{2}$, water, temperature and

[C6a]

nutrient constraints on $V_{\mathrm{b}}$

[C6b]

[C6c]

[C6d]

[C6e]

[C7]

water, temperature and nutrient

[C8a]

constraints on $V_{\mathrm{j}}$

[C8b]

non-stomatal effect related to

[C9]

stomatal effect 
$f_{\mathrm{tbi}}=\exp \left[\boldsymbol{B}_{\mathbf{v}}-\boldsymbol{H}_{\mathrm{av}} /\left(\boldsymbol{R} T_{\mathrm{c} i}\right)\right] /\left\{1+\exp \left[\left(\boldsymbol{H}_{\mathrm{dl}}-\boldsymbol{S} T_{\mathrm{ci}}\right) /\left(\boldsymbol{R} T_{\mathrm{c} i}\right)\right]+\exp \left[\left(\boldsymbol{S} T_{\mathrm{ci}}-\boldsymbol{H}_{\mathrm{dh}}\right) /\left(\boldsymbol{R} T_{\mathrm{c} i}\right)\right]\right\}$

$f_{\mathrm{to} i}=\exp \left[\boldsymbol{B}_{\mathbf{0}}-\boldsymbol{H}_{\mathrm{ao}} /\left(\boldsymbol{R} T_{\mathrm{ci}}\right)\right] /\left\{1+\exp \left[\left(\boldsymbol{H}_{\mathrm{dl}}-\boldsymbol{S} T_{\mathrm{c} i}\right) /\left(\boldsymbol{R} T_{\mathrm{ci}}\right)\right]+\exp \left[\left(\boldsymbol{S} T_{\mathrm{c} i}-\boldsymbol{H}_{\mathbf{d h}}\right) /\left(\boldsymbol{R} T_{\mathrm{c} i}\right)\right]\right]$

$f_{\mathrm{tji}}=\exp \left[\boldsymbol{B}_{\mathbf{j}}-\boldsymbol{H}_{\mathrm{aj}} /\left(\boldsymbol{R} T_{\mathrm{c} i}\right)\right] /\left\{1+\exp \left[\left(\boldsymbol{H}_{\mathbf{d l}}-\boldsymbol{S} T_{\mathrm{c} i}\right) /\left(\boldsymbol{R} T_{\mathrm{c} i}\right)\right]+\exp \left[\left(\boldsymbol{S} T_{\mathrm{c} i}-\boldsymbol{H}_{\mathbf{d h}}\right) /\left(\boldsymbol{R} T_{\mathrm{c} i}\right)\right]\right\}$

$f_{\mathrm{tko} i}=\exp \left[\boldsymbol{B}_{\mathbf{k o}}-\boldsymbol{H}_{\text {ako }} /\left(\boldsymbol{R} T_{\mathrm{c} i}\right)\right]$

$\delta M_{\mathrm{L}_{\mathrm{R} i, j, k}} / \delta t=\delta M_{\mathrm{L}_{i, j, k}} / \delta t \min \left\{\left[\boldsymbol{N}_{\text {leaf }}^{\prime}+\left(\boldsymbol{N}_{\text {leaf }}-\boldsymbol{N}_{\text {leaf }}^{\prime}\right) f_{\mathrm{i} C i}\right] / \mathbf{N}_{\text {prot, }}\left[\boldsymbol{P}_{\text {leaf }}^{\prime}+\left(\boldsymbol{P}_{\text {leaf }}-\boldsymbol{P}_{\text {leaf }}^{\prime}\right) f_{\mathrm{iC} i}\right] / \mathrm{P}_{\text {prot }}\right\}$ $f_{\mathrm{tkci}}=\exp \left[\boldsymbol{B}_{\mathbf{k c}}-\boldsymbol{H}_{\mathrm{akc}} /\left(\boldsymbol{R} T_{\mathrm{ci}}\right)\right]$

$f_{\mathrm{iC} i}=\min \left\{\sigma_{\mathrm{N} i, j} /\left(\sigma_{\mathrm{N} i, j}+\sigma_{\mathrm{C} i, j} / \mathbf{K}_{\mathbf{i} \mathbf{C}_{\mathbf{N}}}\right), \sigma_{\mathrm{P} i, j} /\left(\sigma_{\mathrm{P} i, j}+\sigma_{\mathrm{C} i, j} / K_{\mathbf{i} \mathbf{C}_{\mathbf{P}}}\right)\right\}$

Arrhenius functions for

$[\mathrm{C10a}]$

carboxylation, oxygenation and

electron transport

$[\mathrm{C} 10 \mathrm{~b}]$

temperature sensitivity of $\boldsymbol{K}_{\mathbf{c}_{i}}, \boldsymbol{K}_{\mathbf{o}_{i}}$

[C10c]

$[\mathrm{C} 10 \mathrm{~d}]$

[C10e]

product inhibition of $V_{\mathrm{b}}, V_{\mathrm{j}}$ from

[C11]

$\sigma_{\mathrm{N}}$ and $\sigma_{\mathrm{P}} v s . \sigma_{\mathrm{C}}$ in shoots

leaf structural protein growth

\section{Autotrophic respiration}

$R_{\mathrm{a}}=\Sigma_{i} \Sigma_{, j}\left(R_{\mathrm{c} i, j}+R_{\mathrm{si}, j}\right)+\Sigma_{i} \Sigma_{l} \Sigma_{z}\left(R_{\mathrm{ci}, r, l}+R_{\mathrm{si}, r, l}\right)+\boldsymbol{E}_{\mathrm{N}, \mathrm{P}}\left(U_{\mathrm{NH} 4 i, r, l}+U_{\mathrm{NO} 3 i, r, l}+U_{\mathrm{PO} 4 i, r, l}\right)$

total autotrophic respiration 
$R_{\mathrm{c} i, j}=\boldsymbol{R}_{\mathrm{c}}{ }^{\prime} \sigma_{\mathrm{C} i, j} f_{\mathrm{ta} i}$

$R_{\mathrm{c} i, r, l}=\boldsymbol{R}_{\mathrm{c}}{ }^{\prime} \sigma_{\mathrm{C} i, r, l} f_{\mathrm{ta} i, l}\left(U_{\mathrm{O} 2 i, r, l} / U^{\prime}{ }_{\mathrm{O} 2 i, r, l}\right)$

$U_{\mathrm{O} 2 i, r, l}=U^{\prime}{ }_{\mathrm{O} 2 i, r, l}\left[\mathrm{O}_{2 \mathrm{r}, r, l}\right] /\left(\left[\mathrm{O}_{2 \mathrm{r}, r, l}\right]+\boldsymbol{K}_{\mathbf{O}_{\mathbf{2}}}\right)$

$=U_{\mathrm{w}_{i, r, l}}\left[\mathrm{O}_{2 \mathrm{~s} l}\right]+2 \pi L_{i, r, l} D_{\mathrm{sO} 2}\left(\left[\mathrm{O}_{2 \mathrm{sl}}\right]-\left[\mathrm{O}_{2 \mathrm{ri}, r, l}\right]\right) \ln \left\{\left(r_{\mathrm{s} l}+r_{\mathrm{r} i, r, l}\right) / r_{\mathrm{ri}, r, l}\right\}$

$\left.+2 \pi L_{i, r, l} D_{\mathrm{rO} 2}\left(\left[\mathrm{O}_{2 \mathrm{q} i, r, l}\right]-\left[\mathrm{O}_{2 \mathrm{ri}, r, l}\right]\right) \ln \left(r_{\mathrm{qi}, r, l}\right) / r_{\mathrm{ri}, r, l}\right)$

$U^{\prime}{ }_{\mathrm{O} 2 i, r, l}=2.67 R_{\mathrm{a} i, r, l}^{\prime}$

$R_{\mathrm{s} i, j}=-\min \left\{0.0, R_{\mathrm{c} i, j}-R_{\mathrm{m} i, j}\right\}$

$R_{\mathrm{m} i, j}=\Sigma_{z}\left(\mathbf{N}_{i, j, z} \boldsymbol{R}_{\mathbf{m}}{ }^{\prime} f_{\mathrm{tm} i}\right)$

$R_{\mathrm{g} i, j}=\max \left\{0.0, \min \left\{\left(R_{\mathrm{c} i, j}-R_{\mathrm{m} i, j}\right) \min \left\{1.0, \max \left\{0.0, \psi_{\mathrm{t} i}-\psi_{\mathrm{t}}^{\prime}\right\}\right\}\right.\right.$

\section{Growth and senescence}

$$
l_{i, j, z, \mathrm{C}}=R_{\mathrm{si}, j} M_{\mathrm{L}_{\mathrm{N}} i, j} / M_{\mathrm{L}_{\mathrm{R}} i, j}
$$

$\mathrm{O}_{2}$ constraint on root respiration

[C14a]

from active uptake coupled with

diffusion of $\mathrm{O}_{2}$ from soil as for

[C14b]

heterotrophic respiration in [A17],

and from active uptake coupled

[C14c]

with diffusion of $\mathrm{O}_{2}$ from roots

[C14e]

remobilization when $R_{\mathrm{m}}>R_{\mathrm{c}}$

maintenance respiration

growth when $R_{\mathrm{m}}<R_{\mathrm{c}}$

senescence drives litterfall of non-

remobilizable materia 
$l_{i, j, z, \mathrm{~N}}=l_{i, j, z, \mathrm{C}} \mathbf{N}_{\text {prot }}\left(1.0-\boldsymbol{X}_{\mathbf{m x}} f_{\mathrm{xN} i, j}\right)$

$l_{i, j, z, \mathrm{P}}=l_{i, j, z, \mathrm{C}} \mathbf{P}_{\text {prot }}\left(1.0-\boldsymbol{X}_{\mathbf{m x}} f_{\mathrm{xP} i, j}\right)$

$f_{\mathrm{xN} i, j}=\sigma_{\mathrm{C} i, j} /\left(\sigma_{\mathrm{C} i, j}+\sigma_{\mathrm{N} i, j} / \boldsymbol{K}_{\mathbf{x N}}\right)$

$f_{\mathrm{xP} i, j}=\sigma_{\mathrm{C} i, j} /\left(\sigma_{\mathrm{C} i, j}+\sigma_{\mathrm{P} i, j} / \boldsymbol{K}_{\mathbf{x P}}\right)$

$\delta M_{\mathrm{B} i, j} / \delta t=\Sigma_{z}\left[R_{\mathrm{g} i, j}\left(1-\boldsymbol{Y}_{\mathrm{g} i, z}\right) / \boldsymbol{Y}_{\mathrm{g} i, z}\right]-R_{\mathrm{si}, j}-l_{i, j, \mathrm{C}}$

$\delta M_{\mathrm{R} i, r, l} l \delta t=\left[R_{\mathrm{g}, i, l}\left(1-\boldsymbol{Y}_{\mathrm{g} i, r}\right) / \boldsymbol{Y}_{\mathrm{g} i, r}\right]-R_{\mathrm{s} i, r, l}-l_{i, r, l, \mathrm{C}}$

$\delta A_{\mathrm{L} i, j, k, l} / \delta t=\chi\left(M_{\mathrm{Li}, j, k, l} / y_{i}\right)^{-0.33} \delta M_{\mathrm{Li} i, j, l, l} / \delta t \min \left\{1, \max \left\{0, \psi_{\mathrm{t} i}-\psi_{\mathrm{t}}{ }^{\prime}\right\}\right.$

$\delta L_{i, r, l, l} / \delta \mathrm{t}=\left(\delta M_{\mathrm{R} i, r, l, 1} / \delta \mathrm{t}\right) / y_{i} v_{r} /\left\{\boldsymbol{\rho}_{r}\left(1-\boldsymbol{\theta}_{\mathbf{P}, r}\right)\left(\pi r_{\mathrm{r},, r, l, l}^{2}\right)\right\}$

$\delta L_{i, r, l, 2} / \delta \mathrm{t}=\left(\delta M_{\mathrm{R} i, r, l, 2} / \delta \mathrm{t}\right) \mathrm{v}_{r} /\left\{\boldsymbol{\rho}_{r}\left(1-\boldsymbol{\theta}_{\mathbf{P} i, r}\right)\left(\pi r_{\mathrm{r} i, r, l, 2}^{2}\right)\right\}$ litterfall of $\mathrm{N}$ and $\mathrm{P}$ is driven by

$[\mathrm{C} 19 \mathrm{a}]$

that of $\mathrm{C}$ but reduced by

translocation to to $\sigma_{\mathrm{N}}$ and $\sigma_{\mathrm{P}}$

[C19b]

according to ratios of $\sigma_{\mathrm{N}}$ and $\sigma_{\mathrm{P}}$

with $\sigma_{\mathrm{C}}$

$[\mathrm{C} 19 \mathrm{c}]$

branch growth driven by $R_{\mathrm{g}}$

[C20a]

root growth driven by $R_{\mathrm{g}}$

[C20b]

leaf expansion driven by leaf mass

growth

root extension of primary and

[C21b]

secondary axes driven by root

mass growth

[C21c] 


\section{Root and mycorrhizal nutrient uptake}

$U_{\mathrm{NH} 4 i, r, l}=\left\{U_{\mathrm{wi}, r, l}\left[\mathrm{NH}_{4}{ }_{l}^{+}\right]+2 \pi L_{i, r, l} D_{\mathrm{eNH}_{4 l}}\left(\left[\mathrm{NH}_{4}{ }_{l}^{+}\right]-\left[\mathrm{NH}_{4}{ }_{i, r, l}^{+}\right]\right) / \ln \left(d_{i, r, l} / r_{\mathrm{ri}, r, l}\right)\right\}$

$=\boldsymbol{U}_{\mathbf{N H}_{\mathbf{4}}}^{\prime}\left(U_{\mathrm{O} 2 i, r, l} / U^{\prime}{ }_{\mathrm{O} 2 i, r, l}\right) A_{i, r, l}\left(\left[\mathrm{NH}_{4}{ }^{+}, r, l\right]-\left[\mathbf{N H}_{\mathbf{4}}{ }_{\mathrm{mn}}\right]\right) /\left(\left[\mathrm{NH}_{4}{ }_{i, r, l}\right]-\left[\mathbf{N H}_{\mathbf{4}}{ }^{+}{ }_{\mathbf{m n}}\right]+\boldsymbol{K}_{\mathbf{N H}_{\mathbf{4}}}\right) f_{\mathrm{t}_{\mathrm{i}} l} f_{\mathrm{iNi}, r, l}$

$U_{\mathrm{NO} 3 i, r, l}=\left\{U_{\mathrm{wi}, r, l}\left[\mathrm{NO}_{3}^{-}\right]+2 \pi L_{i, r, l} D_{\mathrm{eNO}_{3 l}}\left(\left[\mathrm{NO}_{3}^{-}\right]-\left[\mathrm{NO}_{3}^{-}{ }_{i, r, l}^{-}\right]\right) / \ln \left(d_{i, r, l} / r_{\mathrm{r} i, r, l}\right)\right\}$

$=\boldsymbol{U}_{\mathbf{N O}_{\mathbf{3}}}^{\prime}\left(U_{\mathrm{O} 2 i, r, l} / U^{\prime}{ }_{\mathrm{O} 2 i, r, l}\right) A_{i, r, l}\left(\left[\mathrm{NO}_{3}^{-}{ }_{i, r, l}\right]-\left[\mathbf{N O}_{\mathbf{3}}{ }_{\mathbf{m n}}\right]\right) /\left(\left[\mathrm{NO}_{3}{ }_{i, r, l}^{-}\right]-\left[\mathbf{N O}_{\mathbf{3}}^{-}{ }_{\mathbf{m n}}\right]+\boldsymbol{K}_{\mathrm{NO}_{\mathbf{3}}}\right) f_{\mathrm{t}_{\mathrm{i}} l} f_{\mathrm{iNi}, r, l}$

$U_{\mathrm{PO} 4 i, r, l}=\left\{U_{\mathrm{wi}, r, l}\left[\mathrm{H}_{2} \mathrm{PO}_{4}^{-}\right]+2 \pi L_{i, r, l} D_{\mathrm{ePO}_{4 l}}\left(\left[\mathrm{H}_{2} \mathrm{PO}_{4}^{-}\right]-\left[\mathrm{H}_{2} \mathrm{PO}_{4 i, r, l}^{-}\right]\right) / \ln \left(d_{i, r, l} / r_{\mathrm{r} i, r, l}\right)\right\}$

$=\boldsymbol{U}_{\mathbf{P O}_{\mathbf{4}}}^{\prime}\left(U_{\mathrm{O} 2 i, r, l} / U^{\prime}{ }_{\mathrm{O} 2 i, r, l}\right) A_{i, r, l}\left(\left[\mathrm{H}_{2} \mathrm{PO}_{4}^{-}{ }_{i, r, l}\right]-\left[\mathbf{H}_{\mathbf{2}} \mathbf{P O}_{\mathbf{4}}^{-} \mathbf{m n}\right]\right) /\left(\left[\mathrm{H}_{2} \mathrm{PO}_{4 i, r, l}^{-}\right]-\left[\mathbf{H}_{\mathbf{2}} \mathbf{P O}_{\mathbf{4}}^{-} \mathbf{m n}^{-}\right]+\boldsymbol{K}_{\mathbf{P O}_{\mathbf{4}}}\right) f_{\mathrm{t}_{\mathrm{g} l}} f_{\mathrm{iPi,r}, l}$

root $\mathrm{N}$ and $\mathrm{P}$ uptake from mass

[C23a]

flow + diffusion coupled with

[C23b]

active uptake of $\mathrm{NH}_{4}^{+}, \mathrm{NO}_{3}^{-}$and

$\mathrm{H}_{2} \mathrm{PO}_{4}{ }^{-}$constrained by $\mathrm{O}_{2}$ uptake,

[C23c]

as for microbial $\mathrm{N}$ and $\mathrm{P}$ uptake in

$[\mathrm{C} 23 \mathrm{~d}]$

[A26]

$f_{\mathrm{iN} i, r, l}=\sigma_{\mathrm{Ci}, r, l} /\left(\sigma_{\mathrm{C} i, r, l}+\sigma_{\mathrm{N} i, r, l} / \boldsymbol{K}_{\mathbf{i N}_{\mathbf{C}}}\right)$

product inhibition of $U_{\mathrm{NH} 4}, U_{\mathrm{NO} 3}$

[C23e]

$f_{\mathrm{iP} i, r, l}=\sigma_{\mathrm{C} i, r, l} /\left(\sigma_{\mathrm{Ci}, r, l}+\sigma_{\mathrm{P} i, r, l} / \boldsymbol{K}_{\mathbf{i P}}\right)$

and $U_{\mathrm{PO} 4}$ determined by $\sigma_{\mathrm{N}}$ and $\sigma_{\mathrm{P}}$

[C23f]

s. $\sigma_{\mathrm{C}}$ in roots

$[\mathrm{C} 23 \mathrm{~g}]$

[C23h]

\section{$\mathrm{C}_{4}$ gross primary productivity}

\section{$\mathrm{C}_{4}$ mesophyll}


$G P P=\sum_{i, j, k, l, m, n, o}\left(V_{\mathrm{g}(\mathrm{m} 4) i, j, k, l, m, n, o}=V_{\mathrm{c}(\mathrm{m} 4) i, j, k, l, m, n, o}\right)$

$V_{\mathrm{g}(\mathrm{m} 4) i, j, k, l, m, n, o}=\left(C_{\mathrm{b}}-C_{\mathrm{i}(\mathrm{m} 4) i, j, k, l, m, n, o}\right) / r_{\mathrm{lfi}, j, k, l, m, n, o}$

gaseous diffusion

$V_{\mathrm{c}(\mathrm{m} 4) i, j, k, l, m, n, o}=\min \left\{V_{\mathrm{b}(\mathrm{m} 4) i, j, k, l, m, n, o}, V_{\mathrm{j}(\mathrm{m} 4) i, j, k, l, m, n, o}\right\}$

mesophyll carboxylation

[C26]

$r_{\mathrm{lfi}, j, k, l, m, n, o}=r_{\mathrm{lfmini} i, j, k, l, m, n, o}+\left(r_{\mathrm{lfmax} i}-r_{\mathrm{lfmin} i, j, k, l, m, n, o}\right) e^{\left(-\beta \psi_{\mathrm{ti}}\right)}$

$r_{\mathrm{lfmini} i, j, l, l, m, n, o}=\left(C_{\mathrm{b}}-C_{\mathrm{i}(\mathrm{m} 4)^{\prime}}{ }^{\prime}\right) / V_{\mathrm{c}_{0}(\mathrm{~m} 4) i, j, k, l, m, n, o}$

[C28]

$\left.V_{\mathrm{b}(\mathrm{m} 4) i, j, k, l, m, n, o}=V_{\mathrm{bmax}(\mathrm{m} 4) i, j, k}\left(C_{\mathrm{c}(\mathrm{m} 4) i, j, k, l, m, n, o}-\Gamma_{(\mathrm{m} 4) i, j, k}\right) /\left(C_{\mathrm{c}(\mathrm{m} 4) i, j, k, l, m, n, o}\right)+K_{\mathrm{c}(\mathrm{m} 4)_{i}}\right)$

$\mathrm{CO}_{2}$-limited carboxylation

[C29]

$V_{\mathrm{j}(\mathrm{m} 4) i, j, k, l, m, n, o}=J_{(\mathrm{m} 4) i, j, k, l, m, n, o} Y_{(\mathrm{m} 4) i, j, k, l, m, n, o}$

light-limited carboxylation

$J_{(\mathrm{m} 4) i, j, k, l, m, n, o}=\left(\varepsilon I_{i, l, m, n, o}+J_{\max (\mathrm{m} 4) i, j, k}-\left(\left(\varepsilon I_{i, l, m, n, o}+J_{\max (\mathrm{m} 4) i, j, k}\right)^{2}-4 \alpha \varepsilon I_{i, l, m, n, o} J_{\max (\mathrm{m} 4) i, j, k}\right)^{0.5}\right) /(2 \alpha)$

irradiance response function

[C31]

$V_{\mathrm{bmax}(\mathrm{m} 4) i, j, k}=V_{\mathrm{bmax}(\mathrm{m} 4)}{ }^{\prime}\left[N_{\mathrm{pep}(\mathrm{m} 4) i, j, k}\right]^{\prime} N_{\mathrm{lfi}, j, k, k} A_{\mathrm{lfi}, j, k} f_{\mathrm{C}(\mathrm{m} 4) i, j, k} f_{\psi i} f_{\mathrm{tv} i}$

PEPc activity

[C32]

$J_{\max (\mathrm{m} 4) i, j, k}=J_{\max }{ }^{\prime}\left[N_{\mathrm{chl}(\mathrm{m} 4) i, j, k}\right]^{\prime} N_{\mathrm{lfi} i, j, k} A_{\mathrm{lfi} j, j, k} f_{\mathrm{C}(\mathrm{m} 4) i, j, k}, f_{\psi i} f_{\mathrm{tvi}}$

chlorophyll activity

[C33]

$f_{\mathrm{C}(\mathrm{m} 4) i, j, k}=1.0 /\left(1.0+\left[\chi_{\mathrm{C} 4(\mathrm{~m} 4) i, j, k}\right] / K_{\left.\mathrm{I} \chi_{\mathrm{C} 4(\mathrm{~m} 4)}\right)}\right.$

$\mathrm{C}_{4}$ product inhibition

[C34]

$f_{\psi i, j, k, l, m, n, o}=\left(r_{\mathrm{lfmin}} i_{i, j, k, l, m, n, o} / r_{\mathrm{If}_{i, j, k, l, m, n, o}}\right)^{0.5}$

non-stomatal water limitation

[C35]

$f_{\mathrm{tv} i}=T_{\mathrm{c} i}\left\{\exp \left[B-H_{\mathrm{a}} /\left(R T_{\mathrm{c} i}\right)\right]\right\} /\left\{1+\exp \left[\left(H_{\mathrm{dl}}-S T_{\mathrm{ci}}\right) /\left(R T_{\mathrm{c} i}\right)\right]+\exp \left[\left(S T_{\mathrm{c} i}-H_{\mathrm{dh}}\right) /\left(R T_{\mathrm{c} i}\right)\right]\right\}$

temperature limitation

\section{$\mathrm{C}_{4}$ mesophyll-bundle sheath exchange}

$V_{\chi \mathrm{C} 4(\mathrm{~m} 4) i, j, k}=\kappa_{\chi \mathrm{C} 4(\mathrm{~m} 4)}\left(\chi_{\mathrm{C} 4(\mathrm{~m} 4) i, j, k} W_{\mathrm{lf}(\mathrm{b} 4) i, j, k}-\chi_{\mathrm{C} 4(\mathrm{~b} 4) i, j, k} W_{\mathrm{lf}(\mathrm{m} 4) i, j, k}\right) /\left(W_{\mathrm{lf}(\mathrm{b} 4) i, j, k}+W_{\mathrm{lf}(\mathrm{m} 4) i, j, k}\right)$

mesophyll-bundle sheath transfer

[C37]

$V_{\chi \mathrm{C} 4(\mathrm{~b} 4) i, j, k}=\kappa_{\chi \mathrm{C} 4(\mathrm{~b} 4)} \chi_{\mathrm{C} 4(\mathrm{~b} 4) i, j, k} /\left(1.0+C_{\mathrm{c}(\mathrm{b} 4) i, j, k} / K_{\mathrm{I} \chi \mathrm{C} 4(\mathrm{~b} 4)}\right)$

bundle sheath decarboxylation

[C38]

$V_{\phi(\mathrm{b} 4) i, j, k}=\kappa_{\mathrm{Cc}(\mathrm{b} 4)}\left(C_{\mathrm{c}(\mathrm{b} 4) i, j, k}-C_{\mathrm{c}(\mathrm{m} 4) i, j, k}\right)\left(12 \times 10^{-9}\right) W_{\mathrm{lf}(\mathrm{b} 4) i, j, k}$

bundle sheath-mesophyll leakage

[C39] 
$\delta \chi_{\mathrm{C} 4(\mathrm{~m} 4) i, j, k} / \delta t=\Sigma_{l, m, n, o} V_{\mathrm{c}(\mathrm{m} 4) i, j, k, l, m, n, o}-V_{\chi \mathrm{C} 4(\mathrm{~m} 4) i, j, k}$

$\delta \chi_{\mathrm{C} 4(\mathrm{~b} 4) i, j, k} / \delta t=V_{\chi \mathrm{C} 4(\mathrm{~m} 4) i, j, k}-V_{\chi \mathrm{C} 4(\mathrm{~b} 4) i, j, k}$

$\delta C_{\mathrm{c}(\mathrm{b} 4) i, j, k} / \delta t=V_{\chi \mathrm{C} 4(\mathrm{~b} 4) i, j, k}-V_{\phi(\mathrm{b} 4) i, j, k}-\Sigma_{l, m, n, o} V_{\mathrm{c}(\mathrm{b} 4) i, j, k, l, m, n, o}$

\section{$\mathrm{C}_{4}$ bundle sheath}

$V_{\mathrm{c}(\mathrm{b} 4) i, j, k, l, m, n, o}=\min \left\{V_{\mathrm{b}(\mathrm{b} 4) i, j, k}, V_{\mathrm{j}(\mathrm{b} 4) i, j, k, l, m, n, o}\right\}$

$\left.V_{\mathrm{b}(\mathrm{b} 4) i, j, k}=V_{\mathrm{bmax}(\mathrm{b} 4) i, j, k}\left(C_{\mathrm{c}(\mathrm{b} 4) i, j, k}-\Gamma_{(\mathrm{b} 4) i, j, k}\right) /\left(C_{\mathrm{c}(\mathrm{b} 4) i, j, k}\right)+K_{\mathrm{c}(\mathrm{b} 4) i}\right)$

$V_{\mathrm{j}(\mathrm{b} 4) i, j, k, l, m, n, o}=J_{(\mathrm{b} 4) i, j, k, l, m, n, o} Y_{(\mathrm{b} 4) i, j, k}$

$J_{(\mathrm{b} 4) i, j, k, l, m, n, o}=\left(\varepsilon I_{i, l, m, n, o}+J_{\max (\mathrm{b} 4) i, j, j, k}-\left(\left(\varepsilon I_{i, l, m, n, o}+J_{\max (\mathrm{b} 4) i, j, k}\right)^{2}-4 \alpha \varepsilon I_{i, l, m, n, o} J_{\max (\mathrm{b} 4) i, j, k}\right)^{0.5}\right) /(2 \alpha)$

$V_{\mathrm{bmax}(\mathrm{b} 4) i, j, k}=V_{\mathrm{bmax}(\mathrm{b} 4)^{\prime}}\left[N_{\mathrm{rub}(\mathrm{b} 4) i, j, k}\right]^{\prime} N_{\mathrm{lfi}, j, k} A_{\mathrm{lfi} i, j, k} f_{\mathrm{C}(\mathrm{c} 3) i, j, k} f_{\psi i} f_{\mathrm{tv} i}$

$J_{\max (\mathrm{b} 4) i, j, k}=J_{\max }{ }^{\prime}\left[N_{\mathrm{chl}(\mathrm{b} 4) i, j, k}\right]^{\prime} N_{\mathrm{lfi}, j, k} A_{\mathrm{lfi} i, j, k} f_{\mathrm{C}(\mathrm{c} 3) i, j, k} f_{\psi i} f_{\mathrm{tv} i}$

$f_{\mathrm{C}(\mathrm{c} 3) i, j, k}=\min \left\{\left[\nu_{\mathrm{lfi} i j}\right] /\left(\left[\nu_{\mathrm{lfi} i, j}\right]+\left[\chi_{\mathrm{c} 3(\mathrm{~b} 4) i, j}\right] / K_{\mathrm{Iv} v_{\mathrm{lf}}}\right),\left[\pi_{\mathrm{ffi}, j}\right] /\left(\left[\pi_{\mathrm{ffi}, j}\right]+\left[\chi_{\mathrm{c} 3(\mathrm{~b} 4) i, j}\right] / K_{\mathrm{I} \pi_{\mathrm{lf}}}\right)\right\}$ mesophyll carboxylation products

bundle sheath carboxylation

products

bundle sheath $\mathrm{CO}_{2}$ concentration

bundle sheath carboxylation

$\mathrm{CO}_{2}$-limited carboxylation

light- limited carboxylation

rradiance response function

RuBPc activity

chlorophyll activity

$\mathrm{C}_{3}$ product inhibition

\section{Definition of variables in appendix C}

\begin{tabular}{llcc}
\hline Variable Definition & Unit & Equation & Reference \\
\hline
\end{tabular}


species or functional type: evergreen,

coniferous, deciduous, annual, perennial,

$\mathrm{C}_{3}, \mathrm{C}_{4}$, monocot, dicot, legume etc.

branch or tiller

$k \quad$ Node

soil or canopy layer

$m \quad$ leaf azimuth

n leaf inclination

$o \quad$ leaf exposure (sunlit $v s$. shaded)

$z \quad$ organ including leaf, stem, root,

mycorrhizae

Variables

$[\mathrm{C} 1, \mathrm{C} 6 \mathrm{~b}, \mathrm{C} 6 \mathrm{~d}, \mathrm{C} 8 \mathrm{~b}$

$\mathrm{C} 21, \mathrm{C} 23, \mathrm{C} 32, \mathrm{C} 33$

,C47] 


\begin{tabular}{|c|c|c|c|c|c|}
\hline$B$ & parameter such that $f_{\mathrm{t}}=1.0$ at $T_{c}=298.15 \mathrm{~K}$ & & [C36] & 17.533 & \\
\hline$B_{\mathbf{j}}$ & parameter such that $f_{\mathrm{t} j i}=1.0$ at $T_{\mathrm{c}}=298.15 \mathrm{~K}$ & & {$[\mathrm{C} 10 \mathrm{c}]$} & 17.363 & \\
\hline$B_{\mathrm{kc}}$ & parameter such that $f_{\text {tkci }}=1.0$ at $T_{\mathrm{c}}=298.15 \mathrm{~K}$ & & {$[\mathrm{C} 10 \mathrm{~d}]$} & 22.187 & \\
\hline$B_{\text {ko }}$ & parameter such that $f_{\text {tkoi }}=1.0$ at $T_{\mathrm{c}}=298.15 \mathrm{~K}$ & & {$[\mathrm{C} 10 \mathrm{e}]$} & 8.067 & \\
\hline $\boldsymbol{B}_{\mathbf{0}}$ & parameter such that $f_{\mathrm{toi}}=1.0$ at $T_{\mathrm{c}}=298.15 \mathrm{~K}$ & & {$[\mathrm{C} 10 \mathrm{~b}]$} & 24.221 & \\
\hline $\boldsymbol{B}_{\mathbf{v}}$ & parameter such that $f_{\mathrm{tvi}}=1.0$ at $T_{\mathrm{c}}=298.15 \mathrm{~K}$ & & {$[\mathrm{C} 10 \mathrm{a}, \mathrm{C} 22]$} & 26.238 & \\
\hline$C_{\mathrm{b}}$ & {$\left[\mathrm{CO}_{2}\right]$ in canopy air } & $\mu \mathrm{mol} \mathrm{mol}{ }^{-1}$ & {$[\mathrm{C} 2, \mathrm{C} 5 \mathrm{C} 25, \mathrm{C} 28]$} & & \\
\hline \multirow[t]{2}{*}{$C_{\mathrm{c}(\mathrm{b} 4)}$} & {$\left[\mathrm{CO}_{2}\right]$ in $\mathrm{C}_{4}$ bundle sheath } & $\mu \mathrm{M}$ & {$[\mathrm{C} 38, \mathrm{C} 39, \mathrm{C} 42, \mathrm{C} 4$} & & \\
\hline & & & 4] & & \\
\hline$C_{\mathrm{c}(\mathrm{m} 4)}$ & {$\left[\mathrm{CO}_{2}\right]$ in $\mathrm{C}_{4}$ mesophyll in equilibrium with $C_{\mathrm{i} i, j, k, l, m, n, o}$} & $\mu \mathrm{M}$ & {$[\mathrm{C} 29, \mathrm{C} 39]$} & & \\
\hline$C_{\mathrm{c}}$ & {$\left[\mathrm{CO}_{2}\right]$ in canopy chloroplasts in equilibrium with $C_{\mathrm{i} i, j, k, l, m, n, o}$} & $\mu \mathrm{M}$ & [C6] & & \\
\hline$C_{\mathrm{i}(\mathrm{m} 4)^{\prime}}$ & {$\left[\mathrm{CO}_{2}\right]$ in $\mathrm{C}_{4}$ mesophyll air when $\psi_{\mathrm{c} i}=0$} & $\mu \mathrm{mol} \mathrm{mol}{ }^{-1}$ & [C28] & $0.45 \times C_{\mathrm{b}}$ & \\
\hline$C_{\mathrm{i}(\mathrm{m} 4)}$ & {$\left[\mathrm{CO}_{2}\right]$ in $\mathrm{C}_{4}$ mesophyll air } & $\mu \mathrm{mol} \mathrm{mol}{ }^{-1}$ & [C25] & & \\
\hline $\mathrm{C}_{i, j, z=l}$ & C content of leaf $(z=l)$ & $\mathrm{g} \mathrm{C} \mathrm{m}^{-2}$ & [C18] & & \\
\hline$C_{\mathrm{i}}{ }^{\prime}$ & {$\left[\mathrm{CO}_{2}\right]$ in canopy leaves when $\psi_{\mathrm{c} i}=0$} & $\mu \mathrm{mol} \mathrm{mol}^{-1}$ & [C5] & $0.70 \times C_{\mathrm{b}}$ & Larcher (2001) \\
\hline$C_{\mathrm{i}}$ & {$\left[\mathrm{CO}_{2}\right]$ in canopy leaves } & $\mu \mathrm{mol} \mathrm{mol}{ }^{-1}$ & {$[\mathrm{C} 2]$} & & \\
\hline
\end{tabular}




\begin{tabular}{|c|c|c|c|c|c|}
\hline$D_{\mathrm{e} \mathrm{NH}} 4 l$ & effective dispersivity-diffusivity of $\mathrm{NH}_{4}{ }^{+}$during root uptake & $\mathrm{m}^{2} \mathrm{~h}^{-1}$ & [C23] & & \\
\hline$D_{\mathrm{e} \mathrm{NO}}{ }_{3 l}$ & effective dispersivity-diffusivity of $\mathrm{NO}_{3}^{-}$during root uptake & $m^{2} h^{-1}$ & [C23] & & \\
\hline$D_{\mathrm{e}} \mathrm{PO}_{4 l}$ & $\begin{array}{l}\text { effective dispersivity-diffusivity of } \mathrm{H}_{2} \mathrm{PO}_{4}^{-} \text {during root } \\
\text { uptake }\end{array}$ & $m^{2} h^{-1}$ & [C23] & & \\
\hline$D_{\mathrm{rO} 2}$ & $\begin{array}{l}\text { aqueous diffusivity of } \mathrm{O}_{2} \text { from root aerenchyma to root or } \\
\text { mycorrhizal surfaces }\end{array}$ & $m^{2} h^{-1}$ & {$[\mathrm{C} 14 \mathrm{~d}]$} & & \\
\hline$D_{\mathrm{sO} 2}$ & $\begin{array}{l}\text { aqueous diffusivity of } \mathrm{O}_{2} \text { from soil to root or mycorrhizal } \\
\text { surfaces }\end{array}$ & $m^{2} h^{-1}$ & {$[\mathrm{C} 14 \mathrm{~d}]$} & & \\
\hline$d_{i, r, l}$ & $\begin{array}{l}\text { half distance between adjacent roots assumed equal to } \\
\text { uptake path length }\end{array}$ & $\mathrm{m}$ & [C23] & $\left(\pi L_{s, z} / \Delta \mathrm{z}\right)^{-1 / 2}$ & Grant (1998a) \\
\hline$E_{\mathrm{N}, \mathrm{P}}$ & energy cost of nutrient uptake & $\operatorname{g~C~g~N}^{-1}$ or $\mathrm{P}^{-1}$ & [C13] & 2.15 & Veen $(1981)$ \\
\hline$f_{\mathrm{C}(\mathrm{c} 3)}$ & $\begin{array}{l}\mathrm{C}_{3} \text { product inhibition of RuBP carboxylation activity in } \mathrm{C}_{4} \\
\text { bundle sheath or } \mathrm{C}_{3} \text { mesophyll }\end{array}$ & - & {$[\mathrm{C} 47, \mathrm{C} 48, \mathrm{C} 49]$} & & \\
\hline$f_{\mathrm{C}(\mathrm{m} 4)}$ & $\begin{array}{l}\mathrm{C}_{4} \text { product inhibition of PEP carboxylation activity in } \mathrm{C}_{4} \\
\text { mesophyll }\end{array}$ & - & {$[\mathrm{C} 32, \mathrm{C} 33, \mathrm{C} 34]$} & & \\
\hline$F_{\text {chl }}$ & fraction of leaf protein in chlorophyll & - & {$[\mathrm{C} 8 \mathrm{~b}]$} & 0.025 & \\
\hline$f_{\mathrm{iC}}$ & N,P inhibition on carboxylation, leaf structural N,P growth & - & {$[\mathrm{C} 6 \mathrm{a}, \mathrm{C} 7, \mathrm{C} 11, \mathrm{C} 12]$} & & \\
\hline
\end{tabular}




\begin{tabular}{|c|c|c|c|c|c|}
\hline$f_{\text {iN }}$ & $\mathrm{N}$ inhibition on root $\mathrm{N}$ uptake & - & {$[\mathrm{C} 23 \mathrm{~g}]$} & & \\
\hline$f_{\mathrm{iP}}$ & $\mathrm{P}$ inhibition on root $\mathrm{P}$ uptake & - & [C23h] & & \\
\hline$F_{\text {rubisco }}$ & fraction of leaf protein in rubisco & - & {$[\mathrm{C} 6 \mathrm{~b}, \mathrm{~d}]$} & 0.125 & \\
\hline$f_{\text {ta }}$ & temperature effect on $R_{\mathrm{a} i, j}$ & - & {$[\mathrm{C} 14, \mathrm{C} 22]$} & & \\
\hline$f_{\mathrm{tb}}$ & temperature effect on carboxylation & - & {$[\mathrm{C} 6 \mathrm{~b}, \mathrm{C} 10 \mathrm{a}]$} & & \\
\hline$f_{\mathrm{tg}}$ & $\begin{array}{l}\text { temperature function for root or mycorrhizal growth } \\
\text { respiration }\end{array}$ & dimensionless & [C23] & & \\
\hline$f_{\mathrm{tj}}$ & temperature effect on electron transport & & {$[\mathrm{C} 8 \mathrm{~b}, \mathrm{C} 10 \mathrm{c}]$} & & \\
\hline$f_{\mathrm{tkc}}$ & temperature effect on $\boldsymbol{K}_{\mathbf{c}_{i}}$ & & {$[\mathrm{C} 6 \mathrm{e}, \mathrm{C} 10 \mathrm{~d}]$} & & $\begin{array}{l}\text { Bernacchi et al. } \\
(2001,2003)\end{array}$ \\
\hline$f_{\text {tko }}$ & temperature effect on $\boldsymbol{K}_{\mathbf{o}_{i}}$ & & {$[\mathrm{C} 6 \mathrm{e}, \mathrm{C} 10 \mathrm{e}]$} & & $\begin{array}{l}\text { Bernacchi et al. } \\
(2001,2003)\end{array}$ \\
\hline$f_{\mathrm{tm}}$ & temperature effect on $R_{\mathrm{m} i, j}$ & - & {$[\mathrm{C} 16, \mathrm{C} 22 \mathrm{~b}]$} & $\mathrm{Q}_{10}=2.25$ & \\
\hline$f_{\mathrm{to}}$ & temperature effect on oxygenation & & {$[\mathrm{C} 6 \mathrm{~d}, \mathrm{C} 10 \mathrm{~b}]$} & & \\
\hline$f_{\mathrm{tv}}$ & temperature effect on carboxylation & - & $\begin{array}{l}{[\mathrm{C} 32, \mathrm{C} 33, \mathrm{C} 36, \mathrm{C} 4} \\
7, \mathrm{C} 48]\end{array}$ & & \\
\hline
\end{tabular}




\begin{tabular}{|c|c|c|c|c|c|}
\hline$f_{\mathrm{xN}}$ & fraction of $\boldsymbol{X}_{\mathbf{m x}} \mathrm{N}$ translocated out of leaf or root during & - & {$[\mathrm{C} 19 \mathrm{a}, \mathrm{c}]$} & & \\
\hline & senescence & & & & \\
\hline$f_{\mathrm{xP}}$ & fraction of $\boldsymbol{X}_{\mathbf{m x}} \mathrm{P}$ translocated out of leaf or root during & - & {$[\mathrm{C} 19 \mathrm{~b}, \mathrm{~d}]$} & & \\
\hline & senescence & & & & \\
\hline$f_{\psi i}$ & non-stomatal water effect on carboxylation & - & {$[\mathrm{C} 6 \mathrm{a}, \mathrm{C} 7, \mathrm{C} 9]$} & & Medrano et al. \\
\hline & & & & & (2002) \\
\hline$f_{\psi i}$ & non-stomatal water effect on carboxylation & - & {$[\mathrm{C} 32, \mathrm{C} 33, \mathrm{C} 3$} & & \\
\hline & & & ,C48] & & \\
\hline$H_{\mathrm{a}}$ & energy of activation & $\mathrm{J} \mathrm{mol}^{-1}$ & [C36] & $57.5 \times 10^{3}$ & \\
\hline$H_{\mathrm{aj}}$ & energy of activation for electron transport & $\mathrm{J} \mathrm{mol}^{-1}$ & {$[\mathrm{C} 10 \mathrm{c}]$} & $43 \times 10^{3}$ & Bernacchi et al. \\
\hline & & & & & $(2001,2003)$ \\
\hline$H_{\text {akc }}$ & parameter for temperature sensitivity of $\boldsymbol{K}_{\mathbf{c}_{i}}$ & $\mathrm{~J} \mathrm{~mol}^{-1}$ & {$[\mathrm{C} 10 \mathrm{~d}]$} & $55 \times 10^{3}$ & Bernacchi et al. \\
\hline & & & & & $(2001,2003)$ \\
\hline $\boldsymbol{H}_{\text {ako }}$ & parameter for temperature sensitivity of $\boldsymbol{K}_{\boldsymbol{o}_{i}}$ & $\mathrm{~J} \mathrm{~mol}^{-1}$ & {$[\mathrm{C} 10 \mathrm{e}]$} & $20 \times 10^{3}$ & Bernacchi et al. \\
\hline & & & & & $(2001,2003)$ \\
\hline $\boldsymbol{H}_{\text {ao }}$ & energy of activation for oxygenation & $\mathrm{J} \mathrm{mol}^{-1}$ & {$[\mathrm{C} 10 \mathrm{~b}, \mathrm{C} 22]$} & $60 \times 10^{3}$ & Bernacchi et al. \\
\hline & & & & & $(2001,2003)$ \\
\hline
\end{tabular}




\begin{tabular}{|c|c|c|c|c|c|}
\hline $\boldsymbol{H}_{\mathrm{av}}$ & energy of activation for carboxylation & $\mathrm{J} \mathrm{mol}^{-1}$ & {$[\mathrm{C} 10 \mathrm{a}, \mathrm{C} 22]$} & $65 \times 10^{3}$ & $\begin{array}{l}\text { Bernacchi et al. } \\
(2001,2003)\end{array}$ \\
\hline $\boldsymbol{H}_{\mathrm{dh}}$ & energy of high temperature deactivation & $\mathrm{J} \mathrm{mol}^{-1}$ & {$[\mathrm{C} 10, \mathrm{C} 22]$} & $222.5 \times 10^{3}$ & \\
\hline$H_{\mathrm{dh}}$ & energy of high temperature deactivation & $\mathrm{J} \mathrm{mol}^{-1}$ & [C36] & $220 \times 10^{3}$ & \\
\hline$H_{\mathrm{dl}}$ & energy of low temperature deactivation & $\mathrm{J} \mathrm{mol}^{-1}$ & {$[\mathrm{C} 10, \mathrm{C} 22]$} & $198.0 \times 10^{3}$ & \\
\hline$H_{\mathrm{dl}}$ & energy of low temperature deactivation & $\mathrm{J} \mathrm{mol}^{-1}$ & [C36] & $190 \times 10^{3}$ & \\
\hline$I$ & Irradiance & $\mu \mathrm{mol} \mathrm{m} \mathrm{s}^{-2}$ & {$[\mathrm{C} 8 \mathrm{a}]$,} & & \\
\hline$J_{(\mathrm{b} 4)}$ & electron transport rate in $\mathrm{C}_{4}$ bundle sheath & $\mu \mathrm{mol} \mathrm{m} \mathrm{s}^{-2}$ & {$[\mathrm{C} 45, \mathrm{C} 46]$} & & \\
\hline$J_{(\mathrm{m} 4)}$ & electron transport rate in $\mathrm{C}_{4}$ mesophyll & $\mu \mathrm{mol} \mathrm{m} \mathrm{s}^{-2} \mathrm{~s}^{-1}$ & {$[\mathrm{C} 30, \mathrm{C} 31]$} & & \\
\hline$J$ & electron transport rate in $C_{3}$ mesophyll & $\mu \mathrm{mol} \mathrm{m}{ }^{-2} \mathrm{~s}^{-1}$ & {$[\mathrm{C} 7, \mathrm{C} 8 \mathrm{a}]$} & & \\
\hline$J_{\max }$ & $\begin{array}{l}\text { specific electron transport rate at non-limiting } I \text { and } 25^{\circ} \mathrm{C} \\
\text { when } \psi_{\mathrm{c} i}=0 \text { and nutrients are nonlimiting }\end{array}$ & $\mu \mathrm{mol} \mathrm{g} \mathrm{g}^{-1} \mathrm{~s}^{-1}$ & {$[\mathrm{C} 33, \mathrm{C} 48]$} & 400 & \\
\hline$J_{\max (b 4)}$ & electron transport rate in $\mathrm{C}_{4}$ bundle sheath at non-limiting $I$ & $\mu \mathrm{mol} \mathrm{m} \mathrm{s}^{-2}$ & {$[\mathrm{C} 46, \mathrm{C} 48]$} & & \\
\hline$J_{\max (\mathrm{m} 4)}$ & electron transport rate in $\mathrm{C}_{4}$ mesophyll at non-limiting $I$ & $\mu \mathrm{mol} \mathrm{m} \mathrm{s}^{-2}$ & {$[\mathrm{C} 31, \mathrm{C} 33]$} & & \\
\hline$J_{\max }$ & electron transport rate at non-limiting $I, \psi_{c i}$, temperature and & $\mu \mathrm{mol} \mathrm{m} \mathrm{s}^{-2} \mathrm{~s}^{-1}$ & {$[\mathrm{C} 8 \mathrm{a}, \mathrm{C} 8 \mathrm{~b}]$} & & \\
\hline
\end{tabular}




\begin{tabular}{|c|c|c|c|c|c|}
\hline$K_{\mathrm{c}(\mathrm{b} 4)}$ & $\begin{array}{l}\text { Michaelis-Menten constant for carboxylation in } \mathrm{C}_{4} \text { bundle } \\
\text { sheath }\end{array}$ & $\mu \mathrm{M}$ & [C44] & $\begin{array}{l}30.0 \text { at } 25^{\circ} \mathrm{C} \text { and } \\
\text { zero } \mathrm{O}_{2}\end{array}$ & Lawlor (1993) \\
\hline$K_{\mathrm{c}(\mathrm{m} 4)}$ & $\begin{array}{l}\text { Michaelis-Menten constant for carboxylation in } \mathrm{C}_{4} \\
\text { mesophyll }\end{array}$ & $\mu \mathrm{M}$ & [C29] & 3.0 at $25^{\circ} \mathrm{C}$ & Lawlor (1993) \\
\hline $\boldsymbol{K}_{\mathrm{c}}$ & Michaelis-Menten constant for carboxylation at zero $\mathrm{O}_{2}$ & $\mu \mathrm{M}$ & {$[\mathrm{C6c}, \mathrm{C6e}]$} & 12.5 at $25^{\circ} \mathrm{C}$ & $\begin{array}{l}\text { Farquhar et al. } \\
\text { (1980) }\end{array}$ \\
\hline$K_{\mathrm{c}}$ & Michaelis-Menten constant for carboxylation at ambient $\mathrm{O}_{2}$ & $\mu \mathrm{M}$ & [C6e] & & \\
\hline$K_{\mathrm{iC}_{\mathrm{N}}}$ & inhibition constant for growth in shoots from $\sigma_{\mathrm{C}} v s . \sigma_{\mathrm{N}}$ & $\mathrm{g} \mathrm{C} \mathrm{g} \mathrm{N}^{-1}$ & [C11] & 100 & Grant (1998a) \\
\hline$K_{\mathrm{iC}_{\mathrm{P}}}$ & inhibition constant for growth in shoots from $\sigma_{\mathrm{C}} v s . \sigma_{\mathrm{P}}$ & $\mathrm{g} \mathrm{C} \mathrm{g} \mathrm{P}^{-1}$ & [C11] & 1000 & Grant (1998a) \\
\hline$K_{\mathrm{I} \chi_{\mathrm{C} 4(64)}}$ & $\begin{array}{l}\text { constant for } \mathrm{CO}_{2} \text { product inhibition of } \mathrm{C}_{4} \text { decarboxylation in } \\
\mathrm{C}_{4} \text { bundle sheath }\end{array}$ & $\mu \mathrm{M}$ & [C38] & 1000.0 & \\
\hline$K_{\mathrm{I} \times \mathrm{C} 4(\mathrm{~m} 4)}$ & $\begin{array}{l}\text { constant for } \mathrm{C}_{4} \text { product inhibition of PEP carboxylation } \\
\text { activity in } \mathrm{C}_{4} \text { mesophyll }\end{array}$ & $\mu \mathrm{M}$ & [C34] & $5 \times 10^{6}$ & \\
\hline
\end{tabular}


$K_{\mathrm{IV}_{\mathrm{lf}}}$ constant for $\mathrm{C}_{3}$ product inhibition of RuBP carboxylation

activity in $\mathrm{C}_{4}$ bundle sheath or $\mathrm{C}_{3}$ mesophyll caused by

$\left[v_{1 f i, j}\right]$

$K_{\mathrm{I} \pi_{\mathrm{lf}}}$

constant for $\mathrm{C}_{3}$ product inhibition of RuBP carboxylation

$\mathrm{g} \mathrm{C} \mathrm{g} \mathrm{P} \mathrm{P}^{-1}$

[C49]

1000

activity in $\mathrm{C}_{4}$ bundle sheath or $\mathrm{C}_{3}$ mesophyll caused by

$\left[\pi_{\mathrm{ffi}, j}\right]$

$K_{\mathbf{i N}_{\mathbf{C}}}$

inhibition constant for $\mathrm{N}$ uptake in roots from $\sigma_{\mathrm{C} i, j} v s . \sigma_{\mathrm{N} j}$

$\mathrm{g} \mathrm{N} \mathrm{g} \mathrm{C}$

$[\mathrm{C} 23]$

0.1

[C23]

0.01

inhibition constant for $\mathrm{P}$ uptake in roots from $\sigma_{\mathrm{C} i, j} v s . \sigma_{\mathrm{P} i, j}$

$\mathrm{g} \mathrm{P} \mathrm{g} \mathrm{C}$

$\mathrm{g} \mathrm{N} \mathrm{m}^{-3}$

$[\mathrm{C} 23]$

0.40

surfaces

$\mathrm{g} \mathrm{N} \mathrm{m}^{-3}$

[C23]

0.35

surfaces

$\boldsymbol{K}_{\mathrm{PO}_{4}} \quad \mathrm{M}-\mathrm{M}$ constant for $\mathrm{H}_{2} \mathrm{PO}_{4}^{-}$uptake root or mycorrhizal

$\mathrm{g} \mathrm{P} \mathrm{m}$

[C23]

0.125

surfaces

$\boldsymbol{K}_{\mathrm{O}_{2}}$

Michaelis-Menten constant for root or mycorrhizal $\mathrm{O}_{2}$

$\mathrm{g} \mathrm{m}^{-3}$

$[\mathrm{C} 14 \mathrm{c}]$

.064 arber and

Silberbush (1984)

Barber and

Silberbush (1984)

Grant (1998a)

Barber and

Silberbush (1984)

Griffin (1972)

uptake 


\begin{tabular}{|c|c|c|c|c|c|}
\hline$K_{\mathbf{o}}$ & inhibition constant for $\mathrm{O}_{2}$ in carboxylation & $\mu \mathrm{M}$ & {$[\mathrm{C} 6 \mathrm{c}, \mathrm{C6e}]$} & 500 at $25^{\circ} \mathrm{C}$ & $\begin{array}{l}\text { Farquhar et al. } \\
\text { (1980) }\end{array}$ \\
\hline$K_{\mathrm{xN}}$ & $\begin{array}{l}\text { inhibition constant for remobilization of leaf or root } \mathrm{N} \\
\text { during senescence }\end{array}$ & $\mathrm{g} \mathrm{N} \mathrm{g} \mathrm{C}^{-1}$ & {$[\mathrm{C} 19 \mathrm{c}]$} & 0.1 & \\
\hline$K_{\mathrm{xP}}$ & $\begin{array}{l}\text { inhibition constant for remobilization of leaf or root } \mathrm{P} \\
\text { during senescence }\end{array}$ & $\operatorname{gPg~C^{-1}}$ & {$[\mathrm{C} 19 \mathrm{~d}]$} & 0.01 & \\
\hline$L$ & root length & $\mathrm{m} \mathrm{m}^{-2}$ & {$[\mathrm{C} 14 \mathrm{~d}, \mathrm{C} 21 \mathrm{~b}, \mathrm{C} 23]$} & & \\
\hline$l_{\mathrm{C}}$ & C litterfall from leaf or root & $\mathrm{g} \mathrm{C} \mathrm{m}^{-2} \mathrm{~h}^{-1}$ & {$[\mathrm{C} 18, \mathrm{C} 19 \mathrm{a}, \mathrm{b}, \mathrm{C} 20]$} & & \\
\hline$l_{\mathrm{N}, \mathrm{P}}$ & $\mathrm{N}$ or $\mathrm{P}$ litterfall from leaf or root & $\mathrm{g} \mathrm{C} \mathrm{m}^{-2} \mathrm{~h}^{-1}$ & {$[\mathrm{C} 19 \mathrm{a}, \mathrm{b}]$} & & \\
\hline$M_{\mathrm{B}}$ & branch C phytomass & $\mathrm{g} \mathrm{C} \mathrm{m}^{-2}$ & [C20] & & \\
\hline$M_{\mathrm{L}}$ & leaf $\mathrm{C}$ phytomass & $\mathrm{g} \mathrm{C} \mathrm{m}^{-2}$ & {$[\mathrm{C} 12, \mathrm{C} 21]$} & & \\
\hline$M_{\mathrm{L}_{\mathrm{N}}}, M_{\mathrm{L}_{\mathrm{R}}}$ & non-remobilizable, remobilizable leaf $\mathrm{C}$ phytomass & $\mathrm{g} \mathrm{C} \mathrm{m}^{-2}$ & {$[\mathrm{C} 12, \mathrm{C} 18]$} & & \\
\hline$M_{\mathrm{R}}$ & root $\mathrm{C}$ phytomass & $\mathrm{g} \mathrm{C} \mathrm{m}^{-2}$ & {$[\mathrm{C} 20, \mathrm{C} 21]$} & & \\
\hline$M_{\text {iprot }}$ & leaf protein phytomass calculated from leaf $\mathrm{N}, \mathrm{P}$ contents & $\mathrm{g} \mathrm{N} \mathrm{m}^{-2}$ & $\begin{array}{l}{[\mathrm{C} 6 \mathrm{~b}, \mathrm{C} 6 \mathrm{~d}, \mathrm{C} 8 \mathrm{~b}, \mathrm{C} 1} \\
2]\end{array}$ & & \\
\hline $\mathrm{N}, \mathrm{P}$ & $\mathrm{N}$ or $\mathrm{P}$ content of organ $z$ & $\mathrm{~g} \mathrm{~N} \mathrm{~m}^{-2}$ & {$[\mathrm{C} 16, \mathrm{C} 19]$} & & \\
\hline $\mathbf{N}_{\text {prot }}$ & $\mathrm{N}$ content of protein remobilized from leaf or root & $\mathrm{g} \mathrm{N} \mathrm{C}^{-1}$ & {$[\mathrm{C} 12, \mathrm{C} 19 \mathrm{a}]$} & 0.4 & \\
\hline
\end{tabular}




\begin{tabular}{|c|c|c|c|c|c|}
\hline$\left[\mathrm{NH}_{4}^{+}{ }_{i, r, l}\right]$ & concentration of $\mathrm{NH}_{4}{ }^{+}$at root or mycorrizal surfaces & $\mathrm{g} \mathrm{N} \mathrm{m}^{-3}$ & [C23] & & \\
\hline \multirow[t]{2}{*}[\mathbf{NH}_{4}{}^{+}{}_{\mathrm{mn}}]{} & concentration of $\mathrm{NH}_{4}{ }^{+}$at root or mycorrizal surfaces below & $\mathrm{g} \mathrm{N} \mathrm{m}^{-3}$ & [C23] & 0.0125 & Barber and \\
\hline & which $U_{\mathrm{NH}_{4}}=0$ & & & & Silberbush (1984) \\
\hline$\left[\mathrm{NO}_{3}^{-} i, r, l\right]$ & concentration of $\mathrm{NH}_{4}^{+}$at root or mycorrizal surfaces & $\mathrm{g} \mathrm{N} \mathrm{m}^{-3}$ & [C23] & & \\
\hline \multirow[t]{2}{*}[\mathrm{NO}_{3}^{-}\mathrm{mn}]{} & concentration of $\mathrm{NO}_{3}{ }^{-}$at root or mycorrizal surfaces below & $\mathrm{g} \mathrm{N} \mathrm{m}^{-3}$ & [C23] & 0.03 & Barber and \\
\hline & which $U_{\mathrm{NO}_{3}}=0$ & & & & Silberbush (1984) \\
\hline$\left[\mathrm{H}_{2} \mathrm{PO}_{4}^{-}{ }_{i, r, l}\right]$ & concentration of $\mathrm{H}_{2} \mathrm{PO}_{4}^{-}$root or mycorrizal surfaces & $\mathrm{g} \mathrm{N} \mathrm{m}^{-3}$ & [C23] & & \\
\hline \multirow[t]{2}{*}[\mathrm{H}_{2}\mathbf{PO}_{4}{}^{-}\mathrm{mn}]{} & concentration of $\mathrm{H}_{2} \mathrm{PO}_{4}^{-}$at root or mycorrizal surfaces below & $\mathrm{g} \mathrm{N} \mathrm{m}^{-3}$ & {$[\mathrm{C} 23]$} & 0.002 & Barber and \\
\hline & which $U_{\mathrm{PO}_{4}}=0$ & & & & Silberbush (1984) \\
\hline$N_{\text {leaf }}$ & maximum leaf structural $\mathrm{N}$ content & $\mathrm{g} \mathrm{N} \mathrm{g} \mathrm{C}^{-1}$ & {$[\mathrm{C} 12]$} & 0.10 & \\
\hline$N_{\text {leaf }}^{\prime}$ & minimum leaf structural $\mathrm{N}$ content & $\mathrm{g} \mathrm{N} \mathrm{g} \mathrm{C}^{-1}$ & {$[\mathrm{C} 12]$} & $0.33 \times N_{\text {leaf }}$ & \\
\hline \multirow[t]{2}{*}{$N_{\text {lf }}$} & total leaf $\mathrm{N}$ & $\mathrm{g} \mathrm{N} \mathrm{m}^{-2}$ leaf & {$[\mathrm{C} 32, \mathrm{C} 33, \mathrm{C} 47, \mathrm{C} 4$} & & \\
\hline & & & $8]$ & & \\
\hline$\left[N_{\mathrm{chl}(b 4)}\right]^{\prime}$ & ratio of chlorophyll $\mathrm{N}$ in $\mathrm{C}_{4}$ bundle sheath to total leaf $\mathrm{N}$ & $\operatorname{g~N~g~N}^{-1}$ & [C48] & 0.05 & \\
\hline$\left[N_{\mathrm{ch}(\mathrm{m} 4)}\right]^{\prime}$ & ratio of chlorophyll $\mathrm{N}$ in $\mathrm{C}_{4}$ mesophyll to total leaf $\mathrm{N}$ & $\mathrm{g} \mathrm{N} \mathrm{g} \mathrm{N}^{-1}$ & [C33] & 0.05 & \\
\hline$\left[N_{\mathrm{pep}(\mathrm{m} 4]^{\prime}}\right.$ & ratio of PEP carboxylase $\mathrm{N}$ in $\mathrm{C}_{4}$ mesophyll to total leaf $\mathrm{N}$ & $\operatorname{g~N~g~N} \mathrm{N}^{-1}$ & [C32] & 0.025 & \\
\hline
\end{tabular}




\begin{tabular}{|c|c|c|c|c|}
\hline$\left[N_{\text {rub(b4) }}\right]^{\prime}$ & ratio of RuBP carboxylase $\mathrm{N}$ in $\mathrm{C}_{4}$ bundle sheath to total & $\operatorname{g~N~g~N}^{-1}$ & [C47] & 0.025 \\
\hline & leaf $\mathrm{N}$ & & & \\
\hline $\mathrm{O}_{2 \mathrm{q}}$ & $\begin{array}{l}\text { aqueous } \mathrm{O}_{2} \text { concentration in root or mycorrhizal } \\
\text { aerenchyma }\end{array}$ & $\mathrm{g} \mathrm{m}^{-3}$ & {$[\mathrm{C} 14 \mathrm{c}, \mathrm{d}]$} & \\
\hline $\mathrm{O}_{2 \mathrm{r}}$ & aqueous $\mathrm{O}_{2}$ concentration at root or mycorrhizal surfaces & $\mathrm{g} \mathrm{m}^{-3}$ & {$[\mathrm{C} 14 \mathrm{c}, \mathrm{d}]$} & \\
\hline $\mathrm{O}_{2 \mathrm{~s}}$ & aqueous $\mathrm{O}_{2}$ concentration in soil solution & $\mathrm{g} \mathrm{m}^{-3}$ & {$[\mathrm{C} 14 \mathrm{c}, \mathrm{d}]$} & \\
\hline$O_{\mathrm{c}}$ & {$\left[\mathrm{O}_{2}\right]$ in canopy chloroplasts in equilibrium with $\mathrm{O}_{2}$ in atm. } & $\mu \mathrm{M}$ & {$[\mathrm{C} 6 \mathrm{c}, \mathrm{C} 6 \mathrm{e}]$} & \\
\hline $\boldsymbol{P}_{\text {leaf }}$ & maximum leaf structural $\mathrm{P}$ content & $\mathrm{g} P \mathrm{~g} \mathrm{C}^{-1}$ & [C12] & 0.10 \\
\hline $\boldsymbol{P}_{\text {leaf }}^{\prime}$ & minimum leaf structural $\mathrm{P}$ content & $\mathrm{g} \mathrm{P} \mathrm{g} \mathrm{C}^{-1}$ & [C12] & $0.33 \times \boldsymbol{P}_{\text {leaf }}$ \\
\hline $\mathbf{P}_{\text {prot }}$ & $\mathrm{P}$ content of protein remobilized from leaf or root & $\mathrm{g} \mathrm{P} \mathrm{C}^{-1}$ & {$[\mathrm{C} 12, \mathrm{C} 19 \mathrm{~b}]$} & 0.04 \\
\hline$\left[\pi_{\mathrm{ff}}\right]$ & concentration of nonstructural root $\mathrm{P}$ uptake product in leaf & $\mathrm{g} \mathrm{P} \mathrm{g} \mathrm{C}^{-1}$ & [C49] & \\
\hline$\theta_{\mathbf{P}}$ & root or mycorrhizal porosity & $\mathrm{m}^{3} \mathrm{~m}^{-3}$ & {$[\mathrm{C} 21 \mathrm{~b}]$} & $0.1-0.5$ \\
\hline $\boldsymbol{R}$ & gas constant & $\mathrm{J} \mathrm{mol}^{-1} \mathrm{~K}^{-1}$ & {$[\mathrm{C} 10, \mathrm{C} 22]$} & 8.3143 \\
\hline$R$ & gas constant & $\mathrm{J} \mathrm{mol}^{-1} \mathrm{~K}^{-1}$ & [C36] & 8.3143 \\
\hline$R_{\mathrm{a}}$ & total autotrophic respiration & $\mathrm{gC} \mathrm{m}^{-2} \mathrm{~h}^{-1}$ & [C13] & \\
\hline$R_{\mathrm{a}}^{\prime}$ & $R_{\mathrm{a}}$ under nonlimiting $\mathrm{O}_{2}$ & $\mathrm{gC} \mathrm{m}^{-2} \mathrm{~h}^{-1}$ & [C14] & \\
\hline
\end{tabular}




\begin{tabular}{|c|c|c|c|c|c|}
\hline $\boldsymbol{R}_{\mathrm{c}}{ }^{\prime}$ & specific autotrophic respiration of $\sigma_{\mathrm{C} i, j}$ at $T_{\mathrm{c} i}=25^{\circ} \mathrm{C}$ & $\mathrm{gC} \mathrm{g} \mathrm{C}^{-1} \mathrm{~h}^{-1}$ & [C14] & 0.015 & \\
\hline \multirow[t]{2}{*}{$R_{\mathrm{c}}$} & autotrophic respiration of $\sigma_{\mathrm{C} i, j}$ or $\sigma_{\mathrm{C} i, r, l}$ & $\mathrm{~g} \mathrm{C} \mathrm{m}^{-2} \mathrm{~h}^{-1}$ & {$[\mathrm{C} 13, \mathrm{C} 14, \mathrm{C} 17$} & & \\
\hline & & & C15] & & \\
\hline$R_{\mathrm{g}}$ & growth respiration & $\mathrm{gC} \mathrm{m}^{-2} \mathrm{~h}^{-1}$ & {$[\mathrm{C} 17, \mathrm{C} 20]$} & & \\
\hline$r_{\mathrm{lf}}$ & leaf stomatal resistance & $\mathrm{s} \mathrm{m}^{-1}$ & {$[\mathrm{C} 25, \mathrm{C} 27, \mathrm{C} 39]$} & & \\
\hline$r_{\text {Ifmax } i}$ & leaf cuticular resistance & $\mathrm{s} \mathrm{m}^{-1}$ & [C27] & & \\
\hline$r_{\mathrm{lfmini} i, j, k, l, m, n, o}$ & leaf stomatal resistance when $\psi_{c i}=0$ & $\mathrm{~s} \mathrm{~m}^{-1}$ & {$[\mathrm{C} 27, \mathrm{C} 28, \mathrm{C} 35$} & & \\
\hline$r_{1, i, j, k, l, m, n, o}$ & leaf stomatal resistance & $\mathrm{s} \mathrm{m}^{-1}$ & {$[\mathrm{C} 2, \mathrm{C} 4, \mathrm{C} 9]$} & & \\
\hline$r_{\max i}$ & leaf cuticular resistance & $\mathrm{s} \mathrm{m}^{-1}$ & [C4] & & \\
\hline$r_{\operatorname{lmin} i, j, k, l, m, n, o}$ & leaf stomatal resistance when $\psi_{c i}=0$ & $\mathrm{~s} \mathrm{~m}^{-1}$ & {$[\mathrm{C} 4, \mathrm{C} 5, \mathrm{C} 9]$} & & \\
\hline $\boldsymbol{R}_{\mathrm{m}}^{\prime}$ & specific maintenance respiration of $\sigma_{\mathrm{C} i, j}$ at $T_{\mathrm{c} i}=25{ }^{\circ} \mathrm{C}$ & $\operatorname{g~Cg~N}^{-1} \mathrm{~h}^{-1}$ & [C16] & 0.0115 & $\begin{array}{l}\text { Barnes et al. } \\
\text { (1998) }\end{array}$ \\
\hline$R_{\mathrm{m} i, j}$ & above-ground maintenance respiration & $\mathrm{gC} \mathrm{m}^{-2} \mathrm{~h}^{-1}$ & {$[\mathrm{C} 16, \mathrm{C} 17, \mathrm{C} 15]$} & & \\
\hline$r_{\mathrm{q}, r, l}$ & radius of root aerenchyma & $\mathrm{m}$ & {$[\mathrm{C} 14 \mathrm{~d}]$} & & \\
\hline$r_{\mathrm{r} i, r, l}$ & root or mycorrhizal radius & $\mathrm{m}$ & {$[\mathrm{C} 14 \mathrm{~d}, \mathrm{C} 21 \mathrm{~b}, \mathrm{c}, \mathrm{C} 23$} & $1.0 \times 10^{-4}$ or $5.0 \times$ & \\
\hline & & & $\mathrm{a}, \mathrm{c}, \mathrm{e}]$ & $10^{-6}$ & \\
\hline
\end{tabular}




\begin{tabular}{|c|c|c|c|c|c|}
\hline$R_{\mathrm{s} i, j}$ & respiration from remobilization of leaf $\mathrm{C}$ & $\mathrm{g} \mathrm{C} \mathrm{m}^{-2} \mathrm{~h}^{-1}$ & $\begin{array}{l}{[\mathrm{C} 13, \mathrm{C} 15, \mathrm{C} 18,} \\
\mathrm{C} 20]\end{array}$ & & \\
\hline$r_{\mathrm{s} l}$ & thickness of soil water films & $\mathrm{m}$ & [C14d] & & \\
\hline $\boldsymbol{\rho}_{r}$ & dry matter content of root biomass & $\mathrm{g} \mathrm{g}^{-1}$ & {$[\mathrm{C} 21 \mathrm{~b}]$} & 0.125 & \\
\hline$S$ & change in entropy & $\mathrm{J} \mathrm{mol}^{-1} \mathrm{~K}^{-1}$ & {$[\mathrm{C} 10, \mathrm{C} 22]$} & 710 & $\begin{array}{l}\text { Sharpe and } \\
\text { DeMichelle } \\
\text { (1977) }\end{array}$ \\
\hline$S$ & change in entropy & $\mathrm{J} \mathrm{mol}^{-1} \mathrm{~K}^{-1}$ & [C36] & 710 & \\
\hline$\sigma_{\mathrm{C}}$ & nonstructural $\mathrm{C}$ product of $\mathrm{CO}_{2}$ fixation & $\mathrm{g} \mathrm{C} \mathrm{g} \mathrm{C}^{-1}$ & $\begin{array}{l}{[\mathrm{C} 11, \mathrm{C} 19 \mathrm{c}, \mathrm{d},} \\
\mathrm{C} 23 \mathrm{~g}, \mathrm{~h}]\end{array}$ & & \\
\hline$\sigma_{\mathrm{N}}$ & nonstructural $\mathrm{N}$ product of root uptake & $\mathrm{g} \mathrm{N} \mathrm{g} \mathrm{C}^{-1}$ & $\begin{array}{l}{[\mathrm{C} 11, \mathrm{C} 19 \mathrm{c},} \\
\mathrm{C} 23 \mathrm{~g}, \mathrm{~h}]\end{array}$ & & \\
\hline$\sigma_{\mathrm{P}}$ & nonstructural $\mathrm{P}$ product of root uptake & $\mathrm{g} \mathrm{P} \mathrm{g} \mathrm{C}^{-1}$ & $\begin{array}{l}{[\mathrm{C} 11, \mathrm{C} 19 \mathrm{~d},} \\
\mathrm{C} 23 \mathrm{~g}, \mathrm{~h}]\end{array}$ & & \\
\hline$T_{\mathrm{c}}$ & canopy temperature & $\mathrm{K}$ & {$[\mathrm{C} 10, \mathrm{C} 22]$} & & \\
\hline$T_{\mathrm{c}}$ & canopy temperature & ${ }^{\circ} \mathrm{C}$ & [C36] & & \\
\hline$U_{\mathrm{NH} 4 i, r, l}$ & $\mathrm{NH}_{4}{ }_{4}^{+}$uptake by roots or mycorrhizae & $\mathrm{g} \mathrm{N} \mathrm{m}^{-2} \mathrm{~h}^{-1}$ & [C23] & & \\
\hline
\end{tabular}




\begin{tabular}{|c|c|c|c|c|c|}
\hline$U^{\prime} \mathrm{NH}_{4}$ & maximum $U_{\mathrm{NH}_{4}}$ at $25^{\circ} \mathrm{C}$ and non-limiting $\mathrm{NH}_{4}{ }^{+}$ & $\mathrm{g} \mathrm{N} \mathrm{m}^{-2} \mathrm{~h}^{-1}$ & [C23] & $5.0 \times 10^{-3}$ & $\begin{array}{l}\text { Barber and } \\
\text { Silberbush (1984) }\end{array}$ \\
\hline$U_{\mathrm{NO} 3 i, r, l}$ & $\mathrm{NO}_{3}{ }^{-}$uptake by roots or mycorrhizae & $\mathrm{g} \mathrm{N} \mathrm{m}^{-2} \mathrm{~h}^{-1}$ & [C23] & & \\
\hline$U^{\prime} \mathrm{NO}_{3}$ & maximum $U_{\mathrm{NO}_{3}}$ at $25^{\circ} \mathrm{C}$ and non-limiting $\mathrm{NO}_{3}^{-}$ & $\mathrm{g} \mathrm{N} \mathrm{m}^{-2} \mathrm{~h}^{-1}$ & [C23] & $5.0 \times 10^{-3}$ & $\begin{array}{l}\text { Barber and } \\
\text { Silberbush (1984) }\end{array}$ \\
\hline$U_{\mathrm{PO} 4 i, r, l}$ & $\mathrm{H}_{2} \mathrm{PO}_{4}^{-}$uptake by roots or mycorrhizae & $\mathrm{g} \mathrm{N} \mathrm{m}^{-2} \mathrm{~h}^{-1}$ & [C23] & & \\
\hline$U^{\prime} \mathrm{PO}_{4}$ & maximum $U_{\mathrm{PO}_{4}}$ at $25{ }^{\circ} \mathrm{C}$ and non-limiting $\mathrm{H}_{2} \mathrm{PO}_{4}^{-}$ & $\mathrm{g} \mathrm{N} \mathrm{m}^{-2} \mathrm{~h}^{-1}$ & [C23] & $5.0 \times 10^{-3}$ & $\begin{array}{l}\text { Barber and } \\
\text { Silberbush (1984) }\end{array}$ \\
\hline$U_{\mathrm{O} 2 i, r, l}$ & $\mathrm{O}_{2}$ uptake by roots and mycorrhizae under ambient $\mathrm{O}_{2}$ & $\mathrm{~g} \mathrm{O} \mathrm{m}{ }^{-2} \mathrm{~h}^{-1}$ & {$[\mathrm{C} 14 \mathrm{~b}, \mathrm{c}, \mathrm{C} 23 \mathrm{~b}, \mathrm{~d}, \mathrm{f}]$} & & \\
\hline$U^{\prime}{ }^{\prime} 2 i, l . r$ & $\mathrm{O}_{2}$ uptake by roots and mycorrhizae under nonlimiting $\mathrm{O}_{2}$ & $\mathrm{~g} \mathrm{O} \mathrm{m}{ }^{-2} \mathrm{~h}^{-1}$ & {$[\mathrm{C} 14 \mathrm{~b}, \mathrm{c}, \mathrm{C} 23 \mathrm{~b}, \mathrm{~d}, \mathrm{f}]$} & & \\
\hline$U_{\mathrm{w}_{i, r}, l}$ & root water uptake & $\mathrm{m}^{3} \mathrm{~m}^{-2} \mathrm{~h}^{-1}$ & {$[\mathrm{C} 14 \mathrm{~d}, \mathrm{C} 23]$} & & \\
\hline$V_{\not(\mathrm{b} 4) i, j, k}$ & $\mathrm{CO}_{2}$ leakage from $\mathrm{C}_{4}$ bundle sheath to $\mathrm{C}_{4}$ mesophyll & $\mathrm{g} \mathrm{C} \mathrm{m}^{-2} \mathrm{~h}^{-1}$ & {$[\mathrm{C} 39, \mathrm{C} 42]$} & & \\
\hline$V_{\mathbf{b}}{ }^{\prime}$ & specific rubisco carboxylation at $25^{\circ} \mathrm{C}$ & $\begin{array}{l}\mu \mathrm{mol} \mathrm{g}{ }^{-1} \text { rubisco } \\
\mathrm{s}^{-1}\end{array}$ & [C6b] & 45 & $\begin{array}{l}\text { Farquhar et al. } \\
\text { (1980) }\end{array}$ \\
\hline$V_{\mathrm{b}(\mathrm{b} 4) i j, j, k}$ & $\mathrm{CO}_{2}$-limited carboxylation rate in $\mathrm{C}_{4}$ bundle sheath & $\mu \mathrm{mol} \mathrm{m}{ }^{-2} \mathrm{~s}^{-1}$ & {$[\mathrm{C} 43, \mathrm{C} 44]$} & & \\
\hline$V_{\mathrm{b}(\mathrm{m} 4) i, j, k, l, m, n, o}$ & $\mathrm{CO}_{2}$-limited carboxylation rate in $\mathrm{C}_{4}$ mesophyll & $\mu \mathrm{mol} \mathrm{m} \mathrm{m}^{-2}$ & {$[\mathrm{C} 26]$} & & \\
\hline$V_{\mathrm{b} i, j, k, l, m, n, o}$ & $\mathrm{CO}_{2}$-limited leaf carboxylation rate & $\mu \mathrm{mol} \mathrm{m} \mathrm{m}^{-2} \mathrm{~s}^{-1}$ & {$[\mathrm{C} 3, \mathrm{C} 6]$} & & \\
\hline
\end{tabular}




\begin{tabular}{|c|c|c|c|c|}
\hline$V_{\mathrm{bmax}(\mathrm{b} 4)^{\prime}}$ & $\begin{array}{l}\text { RuBP carboxylase specific activity in } \mathrm{C}_{4} \text { bundle sheath at } \\
25^{\circ} \mathrm{C} \text { when } \psi_{\mathrm{c} i}=0 \text { and nutrients are nonlimiting }\end{array}$ & $\mu \mathrm{mol} \mathrm{g} \mathrm{g}^{-1} \mathrm{~s}^{-1}$ & [C47] & 75 \\
\hline$V_{\mathrm{bmax}(\mathrm{b} 4), i, j, k}$ & $\mathrm{CO}_{2}$-nonlimited carboxylation rate in $\mathrm{C}_{4}$ bundle sheath & $\mu \mathrm{mol} \mathrm{m} \mathrm{s}^{-2}$ & {$[\mathrm{C} 44, \mathrm{C} 47]$} & \\
\hline$V_{\mathrm{bmax}(\mathrm{m} 4)}{ }^{\prime}$ & $\begin{array}{l}\text { PEP carboxylase specific activity in } \mathrm{C}_{4} \text { mesophyll at } 25^{\circ} \mathrm{C} \\
\text { when } \psi_{\mathrm{ci}}=0 \text { and nutrients are nonlimiting }\end{array}$ & $\mu \mathrm{mol} \mathrm{g} \mathrm{g}^{-1}$ & [C32] & 150 \\
\hline$V_{\mathrm{bmax}(\mathrm{m} 4) i, j, k}$ & $\mathrm{CO}_{2}$-nonlimited carboxylation rate in $\mathrm{C}_{4}$ mesophyll & $\mu \mathrm{mol} \mathrm{m} \mathrm{m}^{-2} \mathrm{~s}^{-1}$ & {$[\mathrm{C} 29, \mathrm{C} 32]$} & \\
\hline$V_{\mathrm{bmax} i, j, k}$ & leaf carboxylation rate at non-limiting $\mathrm{CO}_{2}, \psi_{\mathrm{c} i}, T_{\mathrm{c}}$ and N,P & $\mu \mathrm{mol} \mathrm{m} \mathrm{s}^{-2}$ & {$[\mathrm{C6a}, \mathrm{C} 6 \mathrm{~b}, \mathrm{C} 6 \mathrm{c}]$} & \\
\hline$V_{\mathrm{c}(\mathrm{b} 4) i, j, k, l, m, n, o}$ & $\mathrm{CO}_{2}$ fixation rate in $\mathrm{C}_{4}$ bundle sheath & $\mu \mathrm{mol} \mathrm{m} \mathrm{m}^{-2}$ & [C43] & \\
\hline$V_{\mathrm{c}(\mathrm{m} 4) i, j, k, l, m, n, o}$ & $\mathrm{CO}_{2}$ fixation rate in $\mathrm{C}_{4}$ mesophyll & $\mu \mathrm{mol} \mathrm{m} \mathrm{m}^{-2} \mathrm{~s}^{-1}$ & $\begin{array}{l}{[\mathrm{C} 24, \mathrm{C} 26, \mathrm{C} 40, \mathrm{C} 4} \\
1]\end{array}$ & \\
\hline$V_{\mathrm{c}_{0}(\mathrm{~m} 4) i, j, k, l, m, n, o}$ & $\mathrm{CO}_{2}$ fixation rate in $\mathrm{C}_{4}$ mesophyll when $\psi_{\mathrm{c} i}=0 \mathrm{MPa}$ & $\mu \mathrm{mol} \mathrm{m} \mathrm{m}^{-2} \mathrm{~s}^{-1}$ & [C28] & \\
\hline$V_{\mathrm{ci}, j, k, l, m, n, o}$ & leaf $\mathrm{CO}_{2}$ fixation rate & $\mu \mathrm{mol} \mathrm{m} \mathrm{s}^{-2}$ & {$[\mathrm{C} 1, \mathrm{C} 3]$} & \\
\hline$V_{c^{\prime} i, j, k, l, m, n, o}$ & leaf $\mathrm{CO}_{2}$ fixation rate when $\psi_{\mathrm{c} i}=0$ & $\mu \mathrm{mol} \mathrm{m} \mathrm{m}^{-2} \mathrm{~s}^{-1}$ & {$[\mathrm{C} 5]$} & \\
\hline$V_{\mathrm{g}(\mathrm{m} 4) i, j, k, l, m, n, o}$ & $\mathrm{CO}_{2}$ diffusion rate into $\mathrm{C}_{4}$ mesophyll & $\mu \mathrm{mol} \mathrm{m} \mathrm{s}^{-1}$ & {$[\mathrm{C} 24, \mathrm{C} 25]$} & \\
\hline$V_{\mathrm{g} i, j, k, l, m, n, o}$ & leaf $\mathrm{CO}_{2}$ diffusion rate & $\mu \mathrm{mol} \mathrm{m} \mathrm{s}^{-1}$ & {$[\mathrm{C} 1, \mathrm{C} 2]$} & \\
\hline
\end{tabular}




\begin{tabular}{|c|c|c|c|c|c|}
\hline$V_{\mathrm{j}}^{\prime}$ & specific chlorophyll e transfer at $25^{\circ} \mathrm{C}$ & $\begin{array}{l}\mu \mathrm{mol} \mathrm{g}^{-1} \\
\text { chlorophyll s }\end{array}$ & [C8b] & 450 & $\begin{array}{l}\text { Farquhar et al. } \\
\text { (1980) }\end{array}$ \\
\hline$V_{\mathrm{j}(\mathrm{b} 4), i, j, k, l, m, n, o}$ & irradiance-limited carboxylation rate in $\mathrm{C}_{4}$ bundle sheath & $\mu \mathrm{mol} \mathrm{m} \mathrm{s}^{-1}$ & {$[\mathrm{C} 43, \mathrm{C} 45]$} & & \\
\hline$V_{\mathrm{j}(\mathrm{m} 4) i, j, k, l, m, n, o}$ & irradiance-limited carboxylation rate in $\mathrm{C}_{4}$ mesophyll & $\mu \mathrm{mol} \mathrm{m} \mathrm{s}^{-2}$ & {$[\mathrm{C} 26, \mathrm{C} 30]$} & & \\
\hline$V_{j i, j, k, l, m, n, o}$ & irradiance-limited leaf carboxylation rate & $\mu \mathrm{mol} \mathrm{m} \mathrm{m}^{-2}$ & {$[\mathrm{C} 3, \mathrm{C} 7]$} & & \\
\hline$V_{\mathbf{0}}^{\prime}$ & specific rubisco oxygenation at $25^{\circ} \mathrm{C}$ & $\begin{array}{l}\mu \mathrm{mol} \mathrm{g}{ }^{-1} \text { rubisco } \\
\mathrm{s}^{-1}\end{array}$ & [C6d] & 9.5 & $\begin{array}{l}\text { Farquhar et al. } \\
\text { (1980) }\end{array}$ \\
\hline$V_{\text {omaxi,j,j,k }}$ & leaf oxygenation rate at non-limiting $\mathrm{O}_{2}, \psi_{\mathrm{c} i}, T_{\mathrm{c}}$ and $\mathrm{N}, \mathrm{P}$ & $\mu \mathrm{mol} \mathrm{m} \mathrm{s}^{-1}$ & {$[\mathrm{C} 6 \mathrm{c}, \mathrm{d}]$} & & \\
\hline$V_{\chi C 4(b 4) i, j, k}$ & decarboxylation of $\mathrm{C}_{4}$ fixation product in $\mathrm{C}_{4}$ bundle sheath & $\mathrm{g} \mathrm{C} \mathrm{m}^{-2} \mathrm{~h}^{-1}$ & {$[\mathrm{C} 38, \mathrm{C} 41, \mathrm{C} 42]$} & & \\
\hline$V_{\chi C 4(\mathrm{~m} 4)}$ & $\begin{array}{l}\text { transfer of } \mathrm{C}_{4} \text { fixation product between } \mathrm{C}_{4} \text { mesophyll and } \\
\text { bundle sheath }\end{array}$ & $\mathrm{gC} \mathrm{m}^{-2} \mathrm{~h}^{-1}$ & [C37] & & \\
\hline$\left[\nu_{\mathrm{ff}}\right]$ & concentration of nonstructural root $\mathrm{N}$ uptake product in leaf & $\mathrm{g} \mathrm{N} \mathrm{g} \mathrm{C}^{-1}$ & [C49] & & \\
\hline $\mathrm{v}_{r}$ & specific volume of root biomass & $\mathrm{m}^{3} \mathrm{~g}^{-1}$ & {$[\mathrm{C} 21 \mathrm{~b}]$} & & \\
\hline$W_{\text {lf(b4) }}$ & $\mathrm{C}_{4}$ bundle sheath water content & $\mathrm{g} \mathrm{m}^{-2}$ & {$[$ C37,C39] } & & \\
\hline$W_{\mathrm{lf}(\mathrm{m} 4)}$ & $\mathrm{C}_{4}$ mesophyll water content & $\mathrm{g} \mathrm{m}^{-2}$ & [C37] & & \\
\hline
\end{tabular}




\begin{tabular}{|c|c|c|c|c|c|}
\hline$X_{\mathrm{mx}}$ & $\begin{array}{l}\text { maximum fraction of remobilizable } \mathrm{N} \text { or } \mathrm{P} \text { translocated out } \\
\text { of leaf or root during senescence }\end{array}$ & - & {$[\mathrm{C} 19 \mathrm{a}, \mathrm{b}]$} & 0.6 & Kimmins (2004) \\
\hline$Y_{(\mathrm{b} 4)}$ & $\begin{array}{l}\text { carboxylation yield from electron transport in } \mathrm{C}_{4} \text { bundle } \\
\text { sheath }\end{array}$ & $\begin{array}{l}\mu \mathrm{mol} \mathrm{CO} \\
-1\end{array}$ & [C45] & & \\
\hline$Y_{(\mathrm{m} 4)}$ & carboxylation yield from electron transport in $\mathrm{C}_{4}$ mesophyll & $\underset{-1}{\mu \mathrm{mol} \mathrm{CO}} \mathrm{CO}_{2} \mu \mathrm{mol} \mathrm{e}$ & [C30] & & \\
\hline$Y_{\mathrm{g}}$ & fraction of $\sigma_{\mathrm{C} i, j}$ used for growth expended as $R_{g i, j, z}$ by organ $z$ & $\mathrm{~g} \mathrm{C} \mathrm{g} \mathrm{C}^{-1}$ & [C20] & $\begin{array}{l}0.28(z=\text { leaf }), \\
0.24(z=\text { root and } \\
\text { other non-foliar }) \\
0.20 \text { ( } z=\text { wood })\end{array}$ & $\begin{array}{l}\text { Waring and } \\
\text { Running (1998) }\end{array}$ \\
\hline$y$ & plant population & $\mathrm{m}^{-2}$ & {$[\mathrm{C} 21]$} & & \\
\hline Y & carboxylation yield & $\begin{array}{l}\mu \mathrm{mol} \mathrm{CO} \\
-1\end{array}$ & [C7] & & \\
\hline$\Gamma$ & $\mathrm{CO}_{2}$ compensation point & $\mu \mathrm{M}$ & {$[\mathrm{C} 6 \mathrm{a}, \mathrm{C} 6 \mathrm{c}]$} & & \\
\hline$\Gamma_{(\mathrm{b} 4)}$ & $\mathrm{CO}_{2}$ compensation point in $\mathrm{C}_{4}$ bundle sheath & $\mu \mathrm{M}$ & {$[\mathrm{C} 44]$} & & \\
\hline$\Gamma_{(\mathrm{m} 4)}$ & $\mathrm{CO}_{2}$ compensation point in $\mathrm{C}_{4}$ mesophyll & $\mu \mathrm{M}$ & [C29] & & \\
\hline
\end{tabular}


$\alpha$

$x$

$\chi_{\mathrm{C} 4(\mathrm{~b} 4)}$

$\chi_{\mathrm{C} 4(\mathrm{~m} 4)}$

$\left[\chi_{\mathrm{c} 3(\mathrm{~b} 4)}\right]$

$\left[\chi_{\mathrm{C} 4(\mathrm{~m} 4)}\right]$

$\varepsilon$

$\varepsilon$

$\kappa_{\mathrm{Cc}(\mathrm{b} 4)}$

$\psi_{\mathrm{t}}$ shape parameter for response of $J$ to $I$

shape parameter for response of $J$ to $I$

area:mass ratio of leaf growth

non-structural $\mathrm{C}_{4}$ fixation product in $\mathrm{C}_{4}$ bundle sheath

non-structural $\mathrm{C}_{4}$ fixation product in $\mathrm{C}_{4}$ mesophyll

concentration of non-structural $\mathrm{C}_{3}$ fixation product in $\mathrm{C}_{4}$

bundle sheath

concentration of non-structural $\mathrm{C}_{4}$ fixation product in $\mathrm{C}_{4}$ mesophyll

quantum yield

quantum yield

conductance to $\mathrm{CO}_{2}$ leakage from $\mathrm{C}_{4}$ bundle sheath

canopy turgor potential
$-$

$[\mathrm{C} 8 \mathrm{a}]$

$\mathrm{m} \mathrm{g}^{-3}$

[C31,C46]

[C21]

0.0125

Grant and

Hesketh (1992)

[C37,C38,C41]

$[\mathrm{C} 37, \mathrm{C} 40]$

[C49]

[C34]

$\mu \mathrm{mol} \mathrm{e}^{-} \mu \mathrm{mol}$

[C8a]

quanta $^{-1}$

$\mu \mathrm{mol} \mathrm{e}^{-} \mu \mathrm{mol}$

[C31,C46]

quanta $^{-1}$

$\mathrm{h}^{-1}$

[C39]

20

$\mathrm{MPa}$

[C4]
Farquhar et al

(1980)

Farquhar et al.

(1980) 


\section{Appendix D: Soil water, heat, gas and solute fluxes}

\section{Surface water flux}

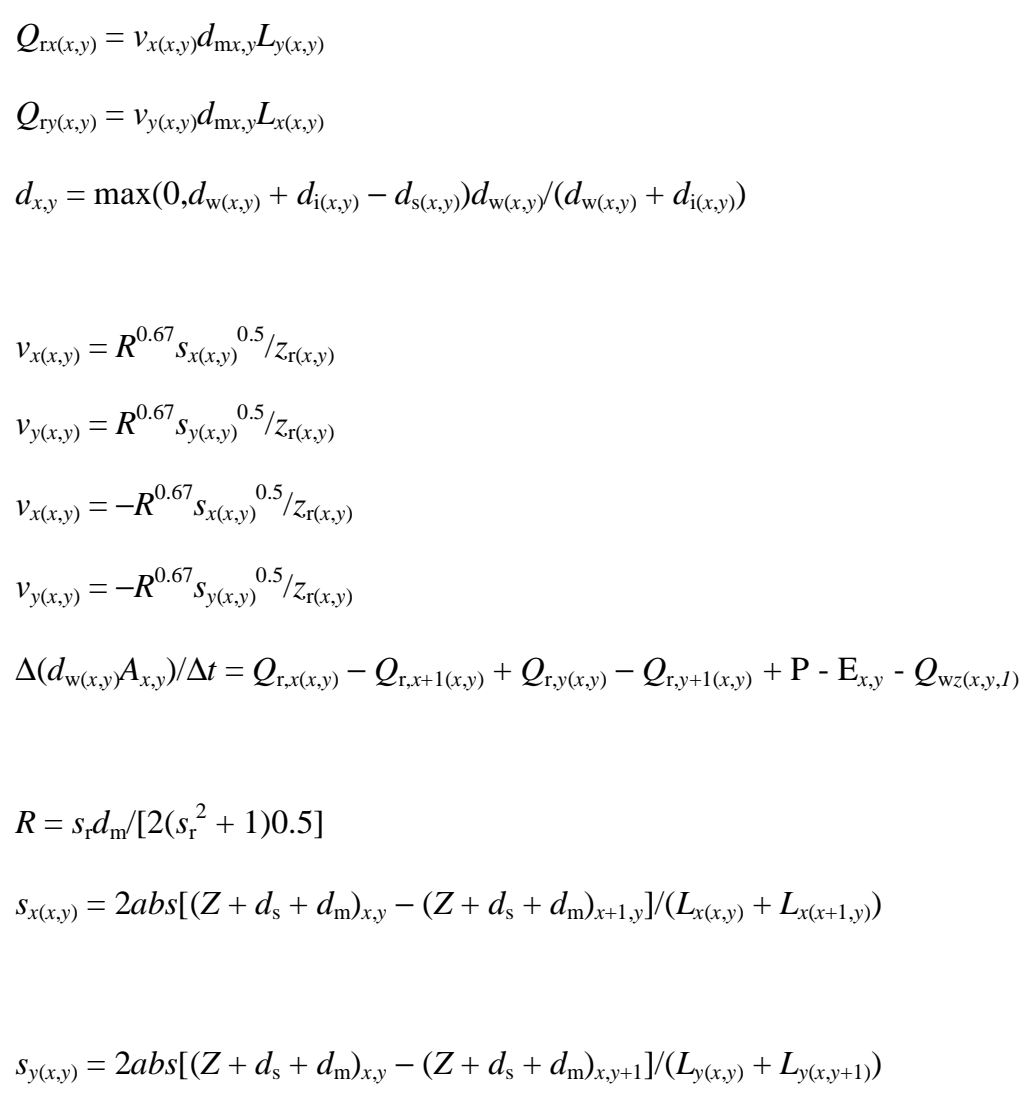

2D Manning equation in $x(\mathrm{EW})$

and $y$ (NS) directions

surface water depth

runoff velocity over E slope

runoff velocity over S slope

runoff velocity over W slope

runoff velocity over $\mathrm{N}$ slope

2D kinematic wave theory for

overland flow

wetted perimeter

2D slope from topography and

pooled surface water in $x(\mathrm{EW})$

and $y(\mathrm{NS})$ directions 


\section{Subsurface water flux}

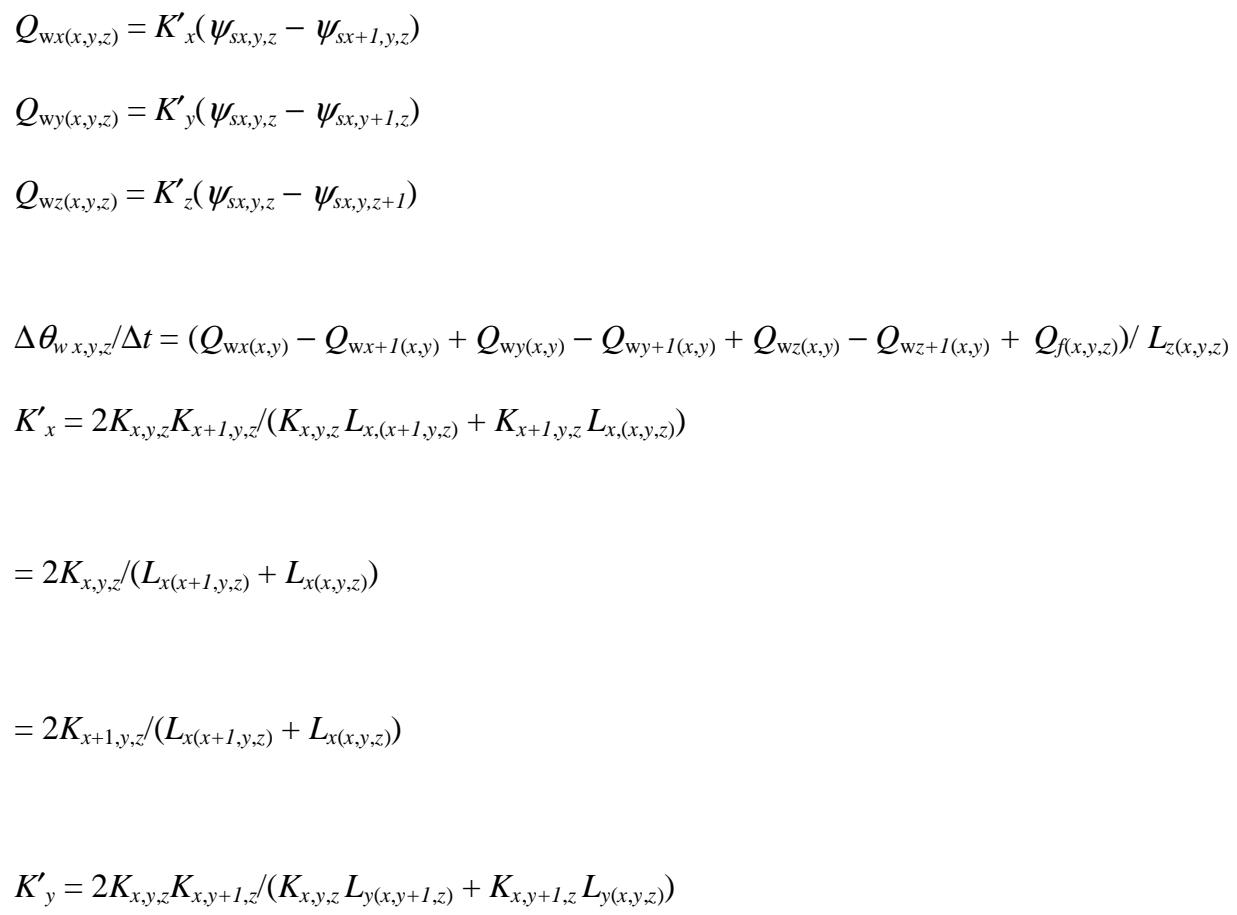

destination cells are unsaturated

in direction $x$ if source cell is

saturated

in direction $x$ if destination cell is

saturated

in direction $y$ if source and

destination cells are unsaturated 
$=2 K_{x, y, z} /\left(L_{y(x, y+1, z)}+L_{y(x, y, z)}\right)$

$=2 K_{x, y+1, z} /\left(L_{y(x, y+1, z)}+L_{y(x, y, z)}\right)$

$K_{z}^{\prime}=2 K_{x, y, z} K_{x, y, z+1} /\left(K_{x, y, z} L_{z(x, y, z+1)}+K_{x, y, z+1} L_{z(x, y, z)}\right)$

$=2 K_{x, y, z} /\left(L_{z(x, y, z+1)}+L_{z(x, y, z)}\right)$

$=2 K_{x, y, z+1} /\left(L_{z(x, y, z+1)}+L_{z(x, y, z)}\right)$

\section{Exchange with water table}

$\operatorname{Qmat}_{\mathrm{tx}(x, y, z)}=\operatorname{Kmat}_{x, y, z}\left[\psi^{\prime}-\psi_{s x, y, z}+0.01\left(d_{z x, y, z}-W T D_{x}\right)\right] /\left(L_{\mathrm{tx}}+0.5 L_{x,(x, y, z)}\right)$

$\operatorname{Qmat}_{\mathrm{t} x(x, y, z)}=\operatorname{Kmat}_{x, y, z}\left[\psi^{\prime}-\psi_{s x, y, z}+0.01\left(d_{z x, y, z}-W T D_{x}\right)\right] /\left(L_{\mathrm{tx}}+0.5 L_{x,(x, y, z)}\right)$ in direction $y$ if source cell is

[D9b]

saturated

in direction $y$ if destination cell is

saturated

in direction $z$ if source and

[D9a]

destination cells are unsaturated

in direction $z$ if source cell is

[D9b]

saturated

in direction $z$ if destination cell is

saturated

if $d_{z x, y, z}<W T D_{x}$ then $\psi_{s x, y, z}>\psi^{\prime}+$

$0.01\left(d_{z x, y, z}-W T D_{x}\right)$ for all depths

$z$ from $d_{z x, y, z}$ to $W T D_{x}$

or if $d_{z x, y, z}>W T D_{x}$ then $\psi_{s x, y, z}>$

$0.01\left(W T D_{x}-d_{z x, y, z}\right)-\psi^{\prime}$ for al

depths $z$ from $W T D_{x}$ to $d_{z x, y, z}$ 
$Q \operatorname{mac} c_{\mathrm{tx}(x, y, z)}=\operatorname{Kmac}_{x, y, z}\left[0.01 * \min \left(0, d_{\mathrm{zx}, y, z}-L_{z(x, y, z)} *\left(\min \left(1, \max \left(0, \theta_{\operatorname{mac}}\right)\right)-0.5\right)-W T D_{x}\right)\right] /\left(L_{\mathrm{t} x}+0.5 L_{x,(x, y, z)}\right)$

$Q m a c_{\mathrm{t} x(x, y, z)}=\operatorname{Kmac}_{x, y, z}\left[0.01 * \max \left(0, d_{\mathrm{zx}, y, z}-L_{z(x, y, z)} *\left(\min \left(1, \max \left(0, \theta_{\operatorname{mac}}\right)\right)-0.5\right)-W T D_{x}\right)\right] /\left(L_{\mathrm{tx}}+0.5 L_{x,(x, y, z)}\right)$

\section{Heat flux}

$R_{n}+\mathbf{L E}+H+G=0$

$G_{x(x, y, z)}=2 \kappa_{(x, y, z),(x+1, y, z)}\left(T_{(x, y, z)}-T_{(x+1, y, z)}\right) /\left(L_{x(x, y, z)}+L_{x(x+1, y, z)}\right)+c_{w} T_{(x, y, z)} Q_{\mathrm{w} x(x, y, z)}$

$G_{y(x, y, z)}=2 \kappa_{(x, y, z),(x, y+1, z)}\left(T_{(x, y, z)}-T_{(x, y+1, z)}\right) /\left(L_{y(x, y, z)}+L_{y(x, y+1, z)}\right)+c_{w} T_{(x, y, z)} Q_{w y(x, y, z)}$

$G_{z(x, y, z)}=2 \kappa_{(x, y, z),(x, y, z+1)}\left(T_{(x, y, z)}-T_{(x, y, z+1)}\right) /\left(L_{z(x, y, z)}+L_{z(x, y, z+1)}\right)+c_{w} T_{(x, y, z)} Q_{\mathrm{w} z(x, y, z)}$

residue and soil layers in $x(\mathrm{EW}), y$ if $d_{z x, y, z}<W T D_{x}$ then $\psi_{s x, y, z}>\psi^{\prime}+$

$0.01\left(d_{z x, y, z}-W T D_{x}\right)$ for all depths

$z$ from $d_{z x, y, z}$ to $W T D_{x}$

or if $d_{z x, y, z}>W T D_{x}$ then $\psi_{s x, y, z}>$

$0.01\left(W T D_{x}-d_{z x, y, z}\right)-\psi^{\prime}$ for all

depths $z$ from $W T D_{x}$ to $d_{z x, y, z}$

[D10a]

for each canopy, snow, residue

and soil surface, depending on

exposure

$3 \mathrm{D}$ conductive - convective heat

[D12]

(NS) and $z$ (vertical) directions 
$G_{x(x-1, y, z)}-G_{x(x, y, z)}+G_{y(x, y-1, z)}-G_{y(x, y, z)}+G_{z(x, y, z-1)}-G_{z(x, y, z)}+\boldsymbol{L} Q_{f(x, y, z)}+c_{(x, y, z)}\left(T_{(x, y, z)}-T_{(x, y, z)}^{\prime}\right) / \Delta t=0$

Gas flux

$Q_{\mathrm{ds} \gamma x, y, z}=a_{\mathrm{gs} x, y, z} D_{\mathrm{d} \gamma}\left(\boldsymbol{S}_{\gamma}^{\prime} \mathrm{ft}_{\mathrm{d} \gamma x, y, z}\left[\gamma_{\mathrm{gs}}\right]_{x, y, z}-\left[\gamma_{\mathrm{ss}}\right]_{x, y, z}\right)$

$Q_{\mathrm{dr} \gamma x, y, z}=a_{\mathrm{gr} x, y, z} D_{\mathrm{d} \gamma}\left(\boldsymbol{S}_{\gamma}^{\prime} f_{\mathrm{d}_{\gamma} x, y, z}\left[\gamma_{\mathrm{gr}}\right]_{x, y, z}-\left[\gamma_{\mathrm{sr}}\right]_{x, y, z}\right)$

$Q_{\mathrm{gs} \gamma z x, y, l}=g_{\mathrm{ax}, y}\left\{\left[\gamma_{\mathrm{a}}\right]-\left\{2\left[\gamma_{\mathrm{gs}}\right]_{x, y, 1} D_{\mathrm{gs} \gamma z(x, y, l)} / L_{\mathrm{z}(x, y, l)}+g_{\mathrm{ax}, y}\left[\gamma_{\mathrm{a}}\right]\right\} /\left\{2 D_{\mathrm{gs} \gamma z(x, y, l)} / L_{\mathrm{z}(x, y, l)}+g_{\mathrm{aa}, y}\right\}\right\}$

$Q_{\mathrm{ds} \gamma x, y, l}=a_{\mathrm{gs} x, y, l} D_{\mathrm{d} \gamma}\left(\boldsymbol{S}_{\gamma}^{\prime} \mathrm{ft}_{\mathrm{d} \gamma x, y, l}\left[\gamma_{\mathrm{a}}\right]-\left[\gamma_{\mathrm{ss}}\right]_{x, y, l}\right)$
$3 \mathrm{D}$ general heat flux equation in

[D13]

snowpack, surface residue and soil

layers

volatilization - dissolution

[D14a]

between aqueous and gaseous

[D14b]

phases in soil and root

volatilization - dissolution

[D15a]

between gaseous and aqueous

phases at the soil surface $(z=1)$

[D15b]

and the atmosphere 


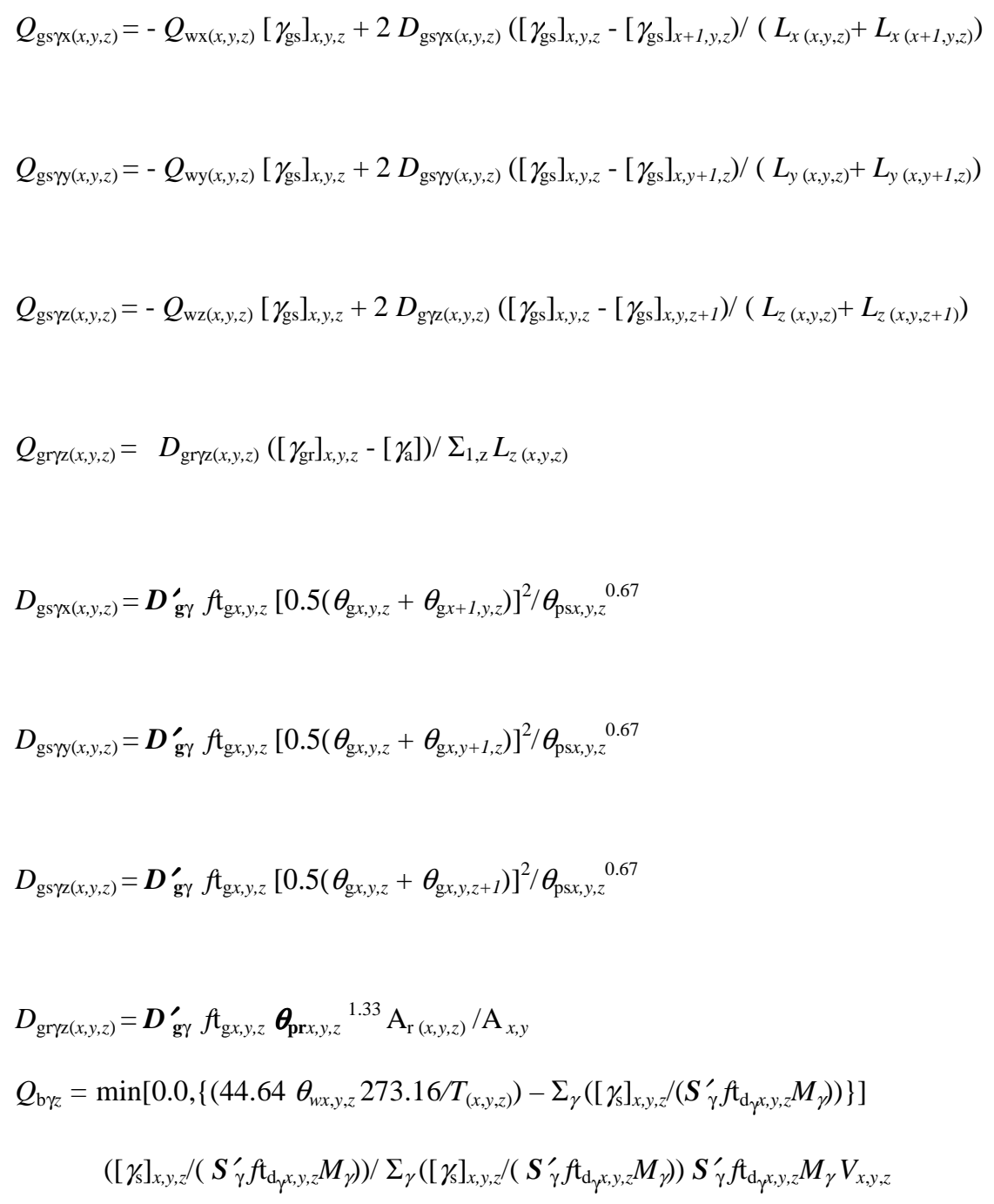

convective - conductive gas

flux between roots and the

[D16d]

atmosphere

gasous diffusivity as a function

of air-filled porosity in soil

gasous diffusivity as a function

of air-filled porosity in roots

$[\mathrm{D} 17 \mathrm{~d}]$

bubbling (-ve flux) when total of

all partial gas pressures exceeds 


\section{Solute flux}

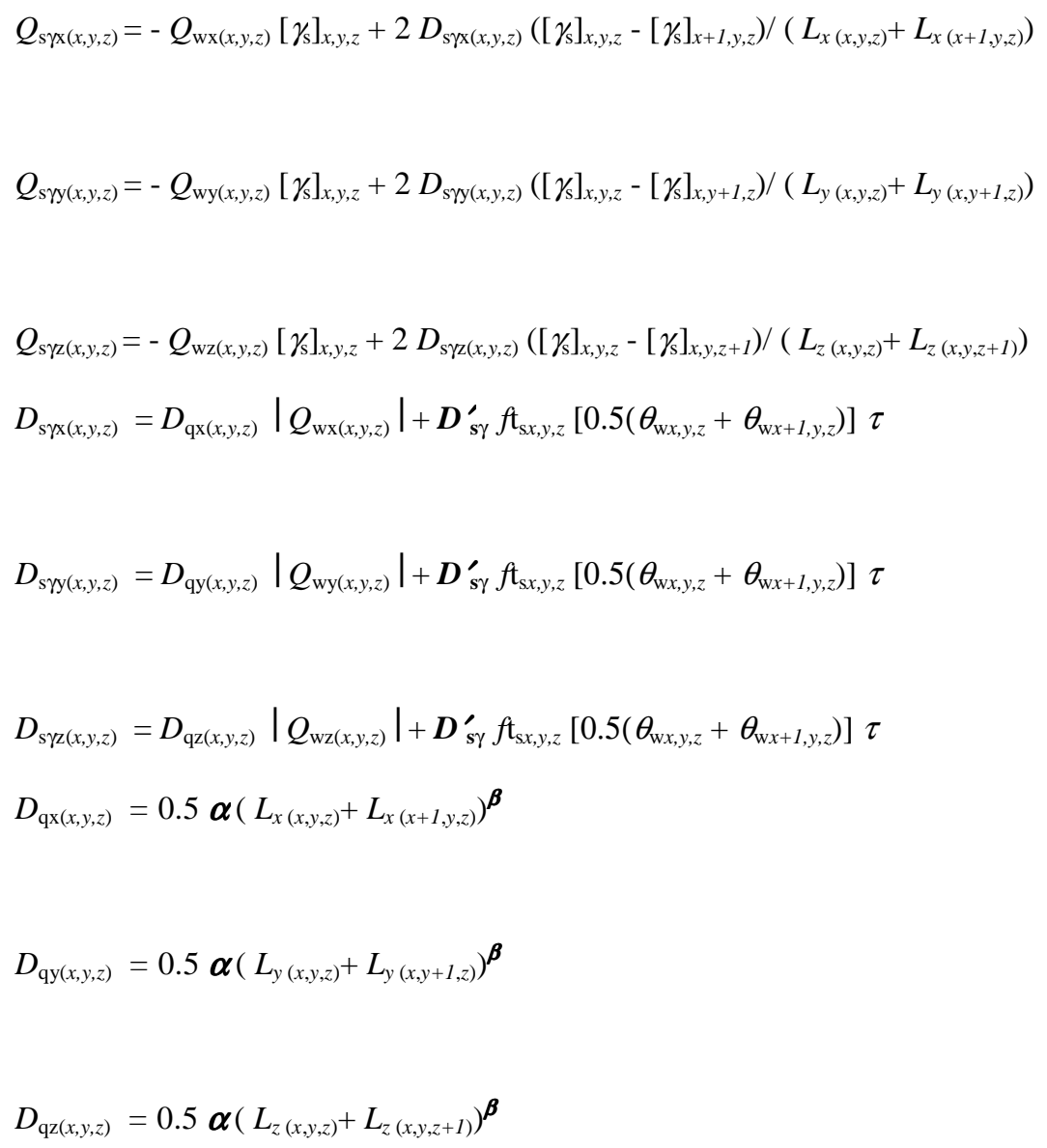

\section{Definition of variables in appendix D}




\begin{tabular}{|c|c|c|c|c|c|}
\hline Variable & Definition & Unit & Equation & Value & Reference \\
\hline \multicolumn{6}{|c|}{ Subscripts } \\
\hline$x$ & grid cell position in west to east direction & & & & \\
\hline$y$ & grid cell position in north to south direction & & & & \\
\hline$z$ & grid cell position in vertical direction & & & $z=0:$ surface & \\
\hline & & & & residue, $\mathrm{z}=1$ to & \\
\hline & & & & $n$ : soil layers & \\
\hline \multicolumn{6}{|c|}{ Variables } \\
\hline$A$ & area of landscape position & $\mathrm{m}^{2}$ & {$[\mathrm{D} 17 \mathrm{c}]$} & & \\
\hline$A_{\mathrm{r}}$ & root cross-sectional area of landscape position & $\mathrm{m}^{2}$ & {$[\mathrm{D} 17 \mathrm{c}]$} & & \\
\hline$a_{\mathrm{gs}}$ & air-water interfacial area in soil & $\mathrm{m}^{2} \mathrm{~m}^{-2}$ & [D14a,D15b] & & Skopp (1985) \\
\hline$\alpha$ & dependence of $D_{\mathrm{q}}$ on $L$ & - & [D21] & & \\
\hline$\beta$ & dependence of $D_{\mathrm{q}}$ on $L$ & - & [D21] & & \\
\hline$c$ & heat capacity of soil & $\mathrm{MJ} \mathrm{m}{ }^{-2} \mathrm{C}^{-1}$ & [D13] & & \\
\hline$c_{w}$ & heat capacity of water & $\mathrm{MJ} \mathrm{m}{ }^{-3}{ }^{\circ} \mathrm{C}^{-1}$ & [D12] & 4.19 & \\
\hline$D_{\mathrm{d} \gamma}$ & volatilization - dissolution transfer coefficient for gas $\gamma$ & $\mathrm{m}^{2} \mathrm{~h}^{-1}$ & {$[\mathrm{D} 14, \mathrm{D} 15 \mathrm{a}]$} & & \\
\hline
\end{tabular}




\begin{tabular}{|c|c|c|c|c|c|}
\hline$D_{\mathrm{gr} \gamma}$ & gaseous diffusivity of gas $\gamma$ in roots & $\mathrm{m}^{2} \mathrm{~h}^{-1}$ & {$[\mathrm{D} 16 \mathrm{~d}, \mathrm{D} 17 \mathrm{~d}]$} & & $\begin{array}{l}\text { Luxmoore et al. } \\
(1970 a, b)\end{array}$ \\
\hline \multirow[t]{2}{*}{$D_{\mathrm{gs \gamma}}$} & gaseous diffusivity of gas $\gamma$ in soil & $\mathrm{m}^{2} \mathrm{~h}^{-1}$ & {$[\mathrm{D} 15 \mathrm{a}, \mathrm{D} 16 \mathrm{a}, \mathrm{b}, \mathrm{c}, \mathrm{D}$} & & Millington and \\
\hline & & & $17 \mathrm{a}, \mathrm{b}, \mathrm{c}]$ & & Quirk (1960) \\
\hline \multirow[t]{2}{*}{$D_{\mathrm{g} \gamma}^{\prime}$} & diffusivity of gas $\gamma$ in air at $0{ }^{\circ} \mathrm{C}$ & $\mathrm{m}^{2} \mathrm{~h}^{-1}$ & {$[\mathrm{D} 17]$} & $6.43 \times 10^{-2}$ for $\gamma=$ & Campbell (1985) \\
\hline & & & & $\mathrm{O}_{2}$ & \\
\hline$D_{\mathrm{q}}$ & dispersivity & $\mathrm{m}$ & {$[\mathrm{D} 20, \mathrm{D} 21]$} & & \\
\hline$D_{\mathrm{s \gamma}}$ & aqueous diffusivity of gas or solute $\gamma$ & $\mathrm{m}^{2} \mathrm{~h}^{-1}$ & {$[\mathrm{D} 19, \mathrm{D} 20]$} & & \\
\hline \multirow[t]{2}{*}{$D_{s \gamma}^{\prime}$} & diffusivity of gas $\gamma$ in water at $0^{\circ} \mathrm{C}$ & $\mathrm{m}^{2} \mathrm{~h}^{-1}$ & {$[\mathrm{D} 20]$} & $8.57 \times 10^{-6}$ for $\gamma=$ & Campbell (1985) \\
\hline & & & & $\mathrm{O}_{2}$ & \\
\hline$d_{\mathrm{m}}$ & depth of mobile surface water & $\mathrm{m}$ & {$[\mathrm{D} 1, \mathrm{D} 2, \mathrm{D} 5 \mathrm{a}, \mathrm{D} 6]$} & & \\
\hline$d_{\mathrm{i}}$ & depth of surface ice & $\mathrm{m}$ & {$[\mathrm{D} 2]$} & & \\
\hline$d_{\mathrm{s}}$ & maximum depth of surface water storage & $\mathrm{m}$ & {$[\mathrm{D} 2, \mathrm{D} 5 \mathrm{~b}]$} & & \\
\hline$W T D_{x}$ & external water table depth & $\mathrm{m}$ & [D10] & & \\
\hline$d_{\mathrm{w}}$ & depth of surface water & $\mathrm{m}$ & {$[\mathrm{D} 1, \mathrm{D} 2]$} & & \\
\hline$d_{z}$ & depth to mid-point of soil layer & $\mathrm{m}$ & [D10] & & \\
\hline E & evaporation or transpiration flux & $\mathrm{m}^{3} \mathrm{~m}^{-2} \mathrm{~h}^{-1}$ & {$[\mathrm{D} 4, \mathrm{D} 11]$} & & \\
\hline$e_{\mathrm{a}}$ & atmospheric vapor density & $\mathrm{m}^{3} \mathrm{~m}^{-3}$ & [D6] & & \\
\hline
\end{tabular}




\begin{tabular}{|c|c|c|c|c|}
\hline$e_{1\left(T_{l}, \psi_{l}\right)}$ & surface litter vapor density at current $T_{1}$ and $\psi_{1}$ & $\mathrm{~g} \mathrm{~m}^{-3}$ & [D6a] & \\
\hline$e_{\mathrm{s}\left(T_{s}, \psi_{s}\right)}$ & soil surface vapor density at current $T_{\mathrm{s}}$ and $\psi_{\mathrm{s}}$ & $\mathrm{g} \mathrm{m}^{-3}$ & [D6b] & \\
\hline$f t_{\mathrm{d}_{\gamma}}$ & temperature dependence of $\boldsymbol{S}_{\gamma}^{\prime}$ & - & [D14,D15b,D18] & $\begin{array}{l}\text { Wilhelm et al. } \\
\text { (1977) }\end{array}$ \\
\hline$f t_{\mathrm{g}}$ & temperature dependence of $\boldsymbol{D}_{\mathrm{g} \gamma}^{\prime}$ & - & [D17] & Campbell (1985) \\
\hline $\mathrm{ft}_{\mathrm{s}}$ & temperature dependence of $\boldsymbol{D}_{\mathrm{s} \gamma}^{\prime}$ & - & [D20] & Campbell (1985) \\
\hline$G$ & soil surface heat flux & $\mathrm{m}^{3} \mathrm{~m}^{-2} \mathrm{~h}^{-1}$ & {$[\mathrm{D} 11]$} & \\
\hline$G_{x}, G_{y}, G_{z}$ & soil heat flux in $x, y$ or $z$ directions & $\mathrm{MJ} \mathrm{m}{ }^{-2} \mathrm{~h}^{-1}$ & {$[\mathrm{D} 12, \mathrm{D} 13]$} & \\
\hline$g_{\mathrm{a}}$ & boundary layer conductance & $\mathrm{m} \mathrm{h}^{-1}$ & {$[\mathrm{D} 15 \mathrm{a}]$} & \\
\hline$\gamma$ & $\begin{array}{l}\text { gas }\left(\mathrm{H}_{2} \mathrm{O}, \mathrm{CO}_{2}, \mathrm{O}_{2}, \mathrm{CH}_{4}, \mathrm{NH}_{3}, \mathrm{~N}_{2} \mathrm{O}, \mathrm{N}_{2}, \mathrm{H}_{2}\right) \text { or solute (from } \\
\text { appendix E) }\end{array}$ & & {$[\mathrm{D} 14, \mathrm{D} 15]$} & \\
\hline$\left[\gamma_{\mathrm{a}}\right]$ & atmospheric concentration of gas $\gamma$ & $\mathrm{g} \mathrm{m}^{-3}$ & {$[\mathrm{D} 15, \mathrm{D} 16 \mathrm{~d}]$} & \\
\hline$\left[\gamma_{\mathrm{gr}}\right]$ & gasous concentration of gas $\gamma$ in roots & $\mathrm{g} \mathrm{m}^{-3}$ & {$[\mathrm{D} 14 \mathrm{~b}, \mathrm{D} 16 \mathrm{~d}]$} & \\
\hline$\left[\gamma_{\mathrm{gs}}\right]$ & gasous concentration of gas $\gamma$ in soil & $\mathrm{g} \mathrm{m}^{-3}$ & $\begin{array}{l}\text { [D14a,D15a,D16a } \\
\text {,D16b,D16c] }\end{array}$ & \\
\hline$\left[\gamma_{\mathrm{sr}}\right]$ & aqueous concentration of gas $\gamma$ in roots & $\mathrm{g} \mathrm{m}^{-3}$ & {$[\mathrm{D} 14 \mathrm{~b}]$} & \\
\hline$\left[\gamma_{s s}\right]$ & aqueous concentration of gas $\gamma$ in soil & $\mathrm{g} \mathrm{m}^{-3}$ & {$[\mathrm{D} 14 \mathrm{a}, \mathrm{D} 15 \mathrm{~b}, \mathrm{D} 18$} & \\
\hline
\end{tabular}




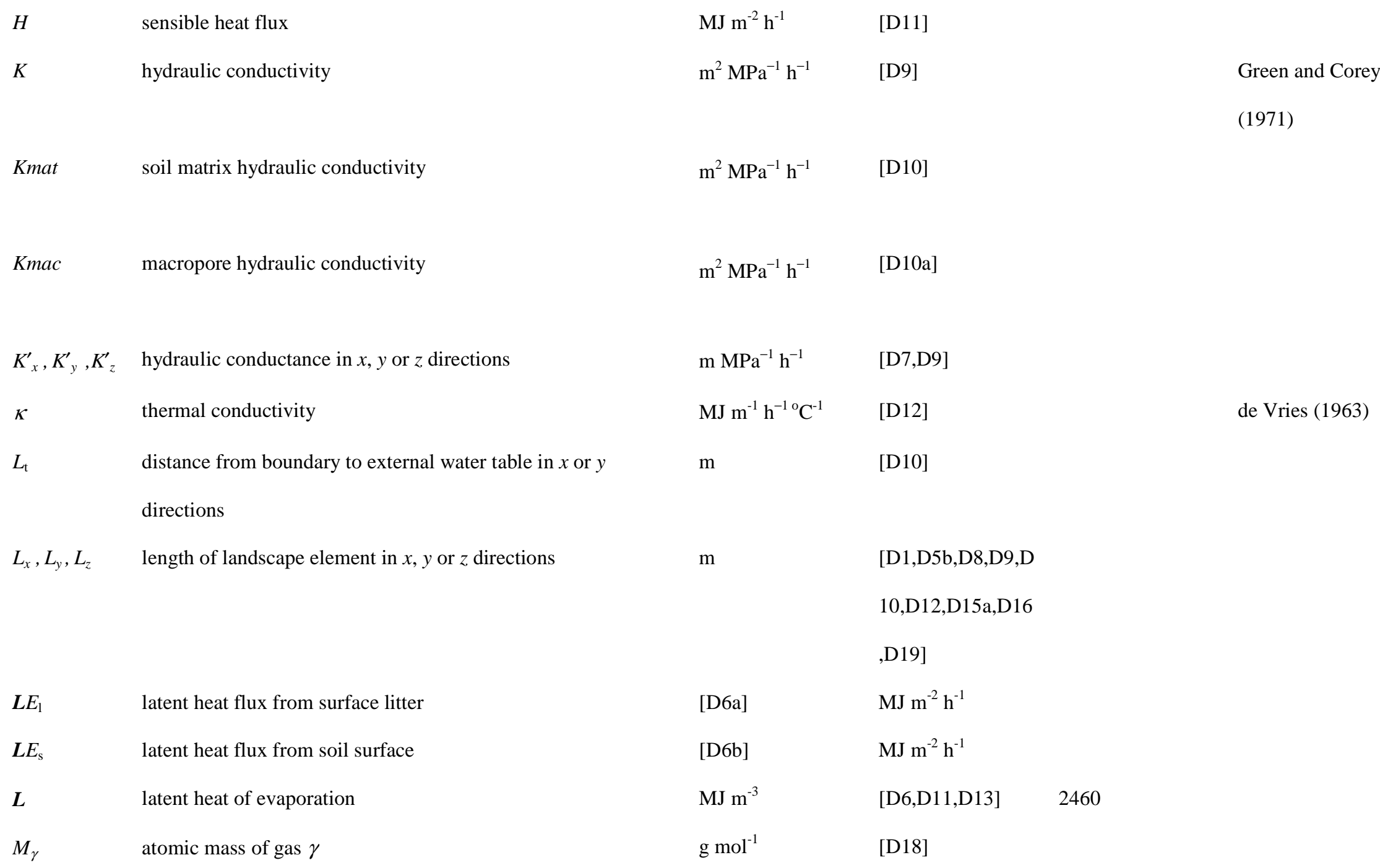




\begin{tabular}{|c|c|c|c|}
\hline$P$ & precipitation flux & $\mathrm{m}^{3} \mathrm{~m}^{-2} \mathrm{~h}^{-1}$ & [D4] \\
\hline$Q_{\mathrm{b} \gamma z}$ & bubbling flux & $\mathrm{g} \mathrm{m}^{-2} \mathrm{~h}^{-1}$ & [D18] \\
\hline$Q_{\mathrm{dr} \gamma}$ & $\begin{array}{l}\text { volatilization - dissolution of gas } \gamma \text { between aqueous and } \\
\text { gaseous phases in roots }\end{array}$ & $\mathrm{g} \mathrm{m}^{-2} \mathrm{~h}^{-1}$ & {$[\mathrm{D} 14 \mathrm{~b}]$} \\
\hline$Q_{\mathrm{ds} \gamma}$ & $\begin{array}{l}\text { volatilization - dissolution of gas } \gamma \text { between aqueous and } \\
\text { gaseous phases in soil }\end{array}$ & $\mathrm{g} \mathrm{m}^{-2} \mathrm{~h}^{-1}$ & {$[\mathrm{D} 14 \mathrm{a}, \mathrm{D} 15 \mathrm{~b}]$} \\
\hline$Q_{f}$ & freeze-thaw flux (thaw +ve) & $\mathrm{m}^{3} \mathrm{~m}^{-2} \mathrm{~h}^{-1}$ & {$[\mathrm{D} 8, \mathrm{D} 13]$} \\
\hline$Q_{\mathrm{gr \gamma}}$ & gaseous flux of gas $\gamma$ between roots and the atmosphere & $\mathrm{g} \mathrm{m}^{-2} \mathrm{~h}^{-1}$ & {$[\mathrm{D} 16 \mathrm{~d}]$} \\
\hline$Q_{\mathrm{gs} \gamma}$ & gaseous flux of gas $\gamma$ in soil & $\mathrm{g} \mathrm{m}^{-2} \mathrm{~h}^{-1}$ & {$[\mathrm{D} 15 \mathrm{a}, \mathrm{D} 16 \mathrm{a}, \mathrm{b}, \mathrm{c}]$} \\
\hline$Q_{\mathrm{r} x}, Q_{\mathrm{ry}}$ & surface water flow in $x$ or $y$ directions & $\mathrm{m}^{3} \mathrm{~m}^{-2} \mathrm{~h}^{-1}$ & {$[\mathrm{D} 1, \mathrm{D} 4]$} \\
\hline$Q_{s \gamma}$ & aqueous flux of gas or solute $\gamma$ & $\mathrm{g} \mathrm{m}^{-2} \mathrm{~h}^{-1}$ & [D19] \\
\hline$Q m a t_{\mathrm{t}}$ & $\begin{array}{l}\text { water flux between boundary grid cell and external water table } \\
\text { through soil matrix in } x \text { or } y \text { directions }\end{array}$ & $\mathrm{m}^{3} \mathrm{~m}^{-2} \mathrm{~h}^{-1}$ & [D10] \\
\hline$Q m a c_{\mathrm{t}}$ & water flux between boundary grid cell and external water table & $m^{3} m^{-2} h^{-1}$ & {$[\mathrm{D} 10 \mathrm{a}]$} \\
\hline
\end{tabular}


$Q_{\mathrm{w} x}, Q_{\mathrm{w} y}, Q_{\mathrm{w} z} \quad$ subsurface water flow in $x, y$ or $z$ directions

$\theta_{\mathrm{g}}$

$\begin{array}{ll}\theta_{\text {mac }} & \text { macropore water content } \\ \boldsymbol{\theta}_{\mathrm{pr}} & \text { root porosity } \\ \theta_{\mathrm{ps}} & \text { soil porosity } \\ \theta_{w} & \text { water-filled porosity } \\ R & \text { ratio of cross-sectional area to perimeter of surface flow } \\ R_{n} & \text { net radiation } \\ r_{\mathrm{a} l} & \text { Surface litter boundary layer resistance } \\ r_{\mathrm{a} s} & \text { Ostwald solubility coefficient of gas } \gamma \text { at } 30{ }^{\circ} \mathrm{C} \\ S_{\gamma}^{\prime} & \\ & \text { slope of channel sides during surface flow } x \text { or } y \text { directions } \\ S_{\mathrm{r}} & \end{array}$

$\begin{array}{ll}\mathrm{m}^{3} \mathrm{~m}^{-2} \mathrm{~h}^{-1} & {[\mathrm{D} 4, \mathrm{D} 7, \mathrm{D} 8, \mathrm{D} 12, \mathrm{D}} \\ & 16, \mathrm{D} 19, \mathrm{D} 20]\end{array}$

$\mathrm{m}^{3} \mathrm{~m}^{-3} \quad[\mathrm{D} 17 \mathrm{a}, \mathrm{b}, \mathrm{c}]$

$\mathrm{m}^{3} \mathrm{~m}^{-3} \quad[\mathrm{D} 10 \mathrm{a}]$

$\mathrm{m}^{3} \mathrm{~m}^{-3} \quad[\mathrm{D} 17 \mathrm{~d}]$

dryland spp. 0.10 Luxmoore et al.

wetland spp. $0.20 \quad(1970 \mathrm{a}, \mathrm{b})$

$\mathrm{m}^{3} \mathrm{~m}^{-3} \quad[\mathrm{D} 17 \mathrm{a}, \mathrm{b}, \mathrm{c}]$

$\mathrm{m}^{3} \mathrm{~m}^{-3} \quad[\mathrm{D} 8, \mathrm{D} 18, \mathrm{D} 20]$

m [D3,D5a]

$\mathrm{MJ} \mathrm{m}^{-2} \mathrm{~h}^{-1} \quad$ [D11]

$\mathrm{m} \mathrm{h}^{-1} \quad$ [D6a]

$\mathrm{m} \mathrm{h}^{-1} \quad$ [D6b]

[D14,D15b,D18] 0.0293 for $\gamma=\mathrm{O}_{2} \quad$ Wilhelm et al.

(1977) $\mathrm{m} \mathrm{m}^{-1} \quad$ [D5a]

$\mathrm{m} \mathrm{m}^{-1} \quad$ [D3,D5b] 
$v_{x}, v_{y} \quad$ velocity of surface flow in $x$ or $y$ directions

\section{Appendix E: Solute transformations}

\section{Precipitation-dissolution equilibria}

\begin{tabular}{|c|c|c|c|}
\hline $\mathrm{Al}(\mathrm{OH})_{3(\mathrm{~s})} \Leftrightarrow\left(\mathrm{Al}^{3+}\right)+3\left(\mathrm{OH}^{-}\right)$ & (amorphous $\left.\mathrm{Al}(\mathrm{OH})_{3}\right)$ & -33.0 & {$[\text { E. } 1]^{1}$} \\
\hline $\mathrm{Fe}(\mathrm{OH})_{3(\mathrm{~s})} \Leftrightarrow\left(\mathrm{Fe}^{3+}\right)+3\left(\mathrm{OH}^{-}\right)$ & (soil Fe) & -39.3 & [E.2] \\
\hline $\mathrm{CaCO}_{3(\mathrm{~s})} \Leftrightarrow\left(\mathrm{Ca}^{2+}\right)+\left(\mathrm{CO}_{3}^{2-}\right)$ & (calcite) & -9.28 & [E.3] \\
\hline $\mathrm{CaSO}_{4(\mathrm{~s})} \Leftrightarrow\left(\mathrm{Ca}^{2+}\right)+\left(\mathrm{SO}_{4}^{2-}\right)$ & (gypsum) & -4.64 & [E.4] \\
\hline $\mathrm{AlPO}_{4(\mathrm{~s})} \Leftrightarrow\left(\mathrm{Al}^{3+}\right)+\left(\mathrm{PO}_{4}^{3-}\right)$ & (variscite) & -22.1 & {$[\text { E. } 5]^{2}$} \\
\hline
\end{tabular}

${ }^{1}$ Round brackets denote solute activity. Numbers in italics denote $\log K$ (precipitation-dissolution, ion pairs), Gapon coefficient (cation exchange) or log $c$ (anion exchange)

${ }^{2}$ All equlilibrium reactions involving $\mathrm{N}$ and $\mathrm{P}$ are calculated for both band and non-band volumes if a banded fertilizer application has been made. These volumes are calculated dynamically from diffusive transport of soluble $\mathrm{N}$ and $\mathrm{P}$. 


$$
\begin{aligned}
& \mathrm{FePO}_{4(\mathrm{~s})} \Leftrightarrow\left(\mathrm{Fe}^{3+}\right)+\left(\mathrm{PO}_{4}^{3-}\right) \\
& \mathrm{Ca}\left(\mathrm{H}_{2} \mathrm{PO}_{4}\right)_{2(\mathrm{~s})} \Leftrightarrow\left(\mathrm{Ca}^{2+}\right)+2\left(\mathrm{H}_{2} \mathrm{PO}_{4}^{-}\right) \\
& \mathrm{CaHPO}_{4(\mathrm{~s})} \Leftrightarrow\left(\mathrm{Ca}^{2+}\right)+\left(\mathrm{HPO}_{4}^{2-}\right) \\
& \mathrm{Ca}_{5}\left(\mathrm{PO}_{4}\right)_{3} \mathrm{OH}_{(\mathrm{s})} \Leftrightarrow 5\left(\mathrm{Ca}^{2+}\right)+3\left(\mathrm{PO}_{4}^{3-}\right)+(\mathrm{OH}) \\
& \mathrm{Cation} \mathrm{exchange} \mathrm{equilibria} \\
& \mathrm{X}-\mathrm{Ca}+2\left(\mathrm{NH}_{4}^{+}\right) \Leftrightarrow 2 \mathrm{X}-\mathrm{NH}{ }_{4}^{+}\left(\mathrm{Ca}^{2+}\right) \\
& 3 \mathrm{X}-\mathrm{Ca}+2\left(\mathrm{Al}^{3+}\right) \Leftrightarrow 2 \mathrm{X}-\mathrm{Al}+3\left(\mathrm{Ca}^{2+}\right) \\
& \mathrm{X}-\mathrm{Ca}+\left(\mathrm{Mg}^{2+}\right) \Leftrightarrow \mathrm{X}-\mathrm{Mg}+\left(\mathrm{Ca}^{2+}\right) \\
& \mathrm{X}-\mathrm{Ca}+2\left(\mathrm{Na}^{+}\right) \Leftrightarrow 2 \mathrm{X}-\mathrm{Na}+\left(\mathrm{Ca}^{2+}\right) \\
& \mathrm{X}-\mathrm{Ca}+2\left(\mathrm{~K}^{+}\right) \Leftrightarrow 2 \mathrm{X}-\mathrm{K}+\left(\mathrm{Ca}^{2+}\right) \\
& \mathrm{X}-\mathrm{Ca}+2\left(\mathrm{H}^{+}\right) \Leftrightarrow 2 \mathrm{X}-\mathrm{H}+\left(\mathrm{Ca}^{2+}\right)
\end{aligned}
$$

\section{Cation exchange equilibria ${ }^{4}$}

\section{Anion adsorption equilibria}

$$
\begin{aligned}
& \mathrm{X}-\mathrm{OH}_{2}^{+} \Leftrightarrow \mathrm{X}-\mathrm{OH}+\left(\mathrm{H}^{+}\right) \\
& \mathrm{X}-\mathrm{OH} \Leftrightarrow \mathrm{X}-\mathrm{O}^{-}+\left(\mathrm{H}^{+}\right)
\end{aligned}
$$

${ }^{3}$ May only be entered as fertilizer, not considered to be naturally present in soils.

${ }^{4} \mathrm{X}$ - denotes surface exchange site for cation or anion adsorption. 
$\mathrm{X}-\mathrm{H}_{2} \mathrm{PO}_{4}+\mathrm{H}_{2} \mathrm{O} \Leftrightarrow \mathrm{X}-\mathrm{OH}_{2}^{+}+\left(\mathrm{H}_{2} \mathrm{PO}_{4}^{-}\right)$

$\mathrm{X}-\mathrm{H}_{2} \mathrm{PO}_{4}+\left(\mathrm{OH}^{-}\right) \Leftrightarrow \mathrm{X}-\mathrm{OH}+\left(\mathrm{H}_{2} \mathrm{PO}_{4}^{-}\right)$

$\mathrm{X}-\mathrm{HPO}_{4}^{-}+\left(\mathrm{OH}^{-}\right) \Leftrightarrow \mathrm{X}-\mathrm{OH}+\left(\mathrm{HPO}_{4}{ }^{2-}\right)$

\section{Organic acid equilibria}

$\mathrm{X}-\mathrm{COOH} \Leftrightarrow \mathrm{X}-\mathrm{COO}^{-}+\left(\mathrm{H}^{+}\right)$

\section{Ion pair equilibria}

$$
\begin{aligned}
& \left(\mathrm{NH}_{4}^{+}\right) \Leftrightarrow\left(\mathrm{NH}_{3}\right)_{(\mathrm{g})}+\left(\mathrm{H}^{+}\right) \\
& \mathrm{H}_{2} \mathrm{O} \Leftrightarrow\left(\mathrm{H}^{+}\right)+\left(\mathrm{OH}^{-}\right) \\
& \left(\mathrm{CO}_{2}\right)_{(\mathrm{g})}+\mathrm{H}_{2} \mathrm{O} \Leftrightarrow\left(\mathrm{H}^{+}\right)+\left(\mathrm{HCO}_{3}^{-}\right) \\
& \left(\mathrm{HCO}_{3}^{-}\right) \Leftrightarrow\left(\mathrm{H}^{+}\right)+\left(\mathrm{CO}_{3}^{2-}\right) \\
& \left(\mathrm{AlOH}^{2+}\right) \Leftrightarrow\left(\mathrm{Al}^{3+}\right)+\left(\mathrm{OH}^{-}\right) \\
& \left(\mathrm{Al}^{2-} \mathrm{OH}_{2}^{+}\right) \Leftrightarrow\left(\mathrm{AlOH}^{2+}\right)+\left(\mathrm{OH}^{-}\right) \\
& \left.\left(\mathrm{Al}^{+} \mathrm{OH}\right)_{3}^{0}\right) \Leftrightarrow\left(\mathrm{Al}\left(\mathrm{OH}_{2}^{+}\right)+\left(\mathrm{OH}^{-}\right)\right. \\
& \left(\mathrm{Al}(\mathrm{OH})_{4}^{-}\right) \Leftrightarrow\left(\mathrm{Al}(\mathrm{OH})_{3}^{0}\right)+\left(\mathrm{OH}^{-}\right) \\
& \left(\mathrm{AlSO}{ }_{4}^{+}\right) \Leftrightarrow\left(\mathrm{Al}^{3+}\right)+\left(\mathrm{SO}_{4}^{2-}\right)
\end{aligned}
$$

$\begin{array}{ll}-9.24 & {[\text { E.22] }} \\ -14.3 & {[\text { E.23] }} \\ -6.42 & {[\text { E.24] }} \\ -10.4 & {[\text { E.25] }} \\ -9.06 & {[\text { E.26] }} \\ -10.7 & {[\text { E.27] }} \\ -5.70 & {[\text { E.28 }]} \\ -5.10 & {[\text { E.29] }} \\ -3.80 & {[\text { E.30] }}\end{array}$




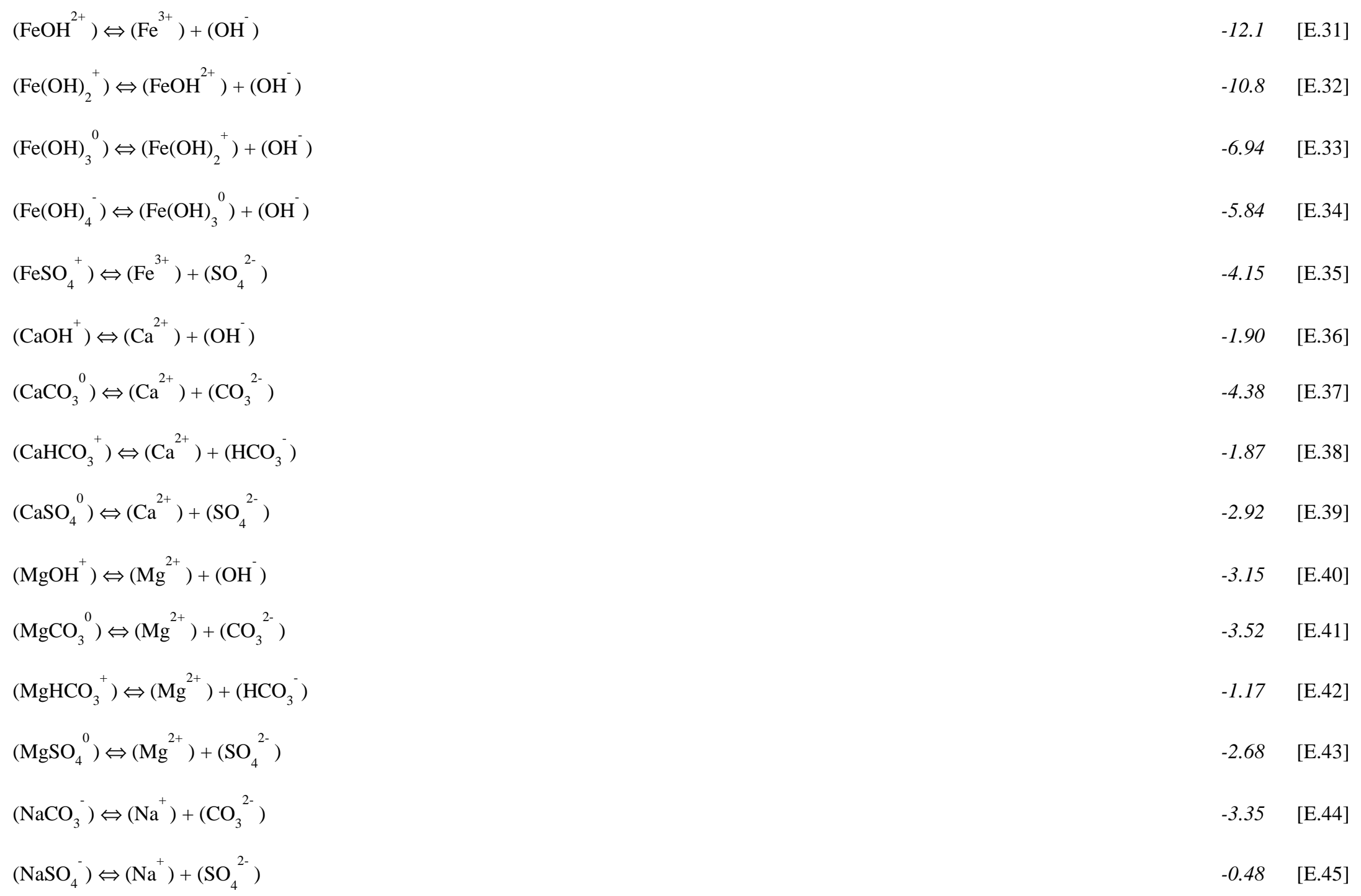




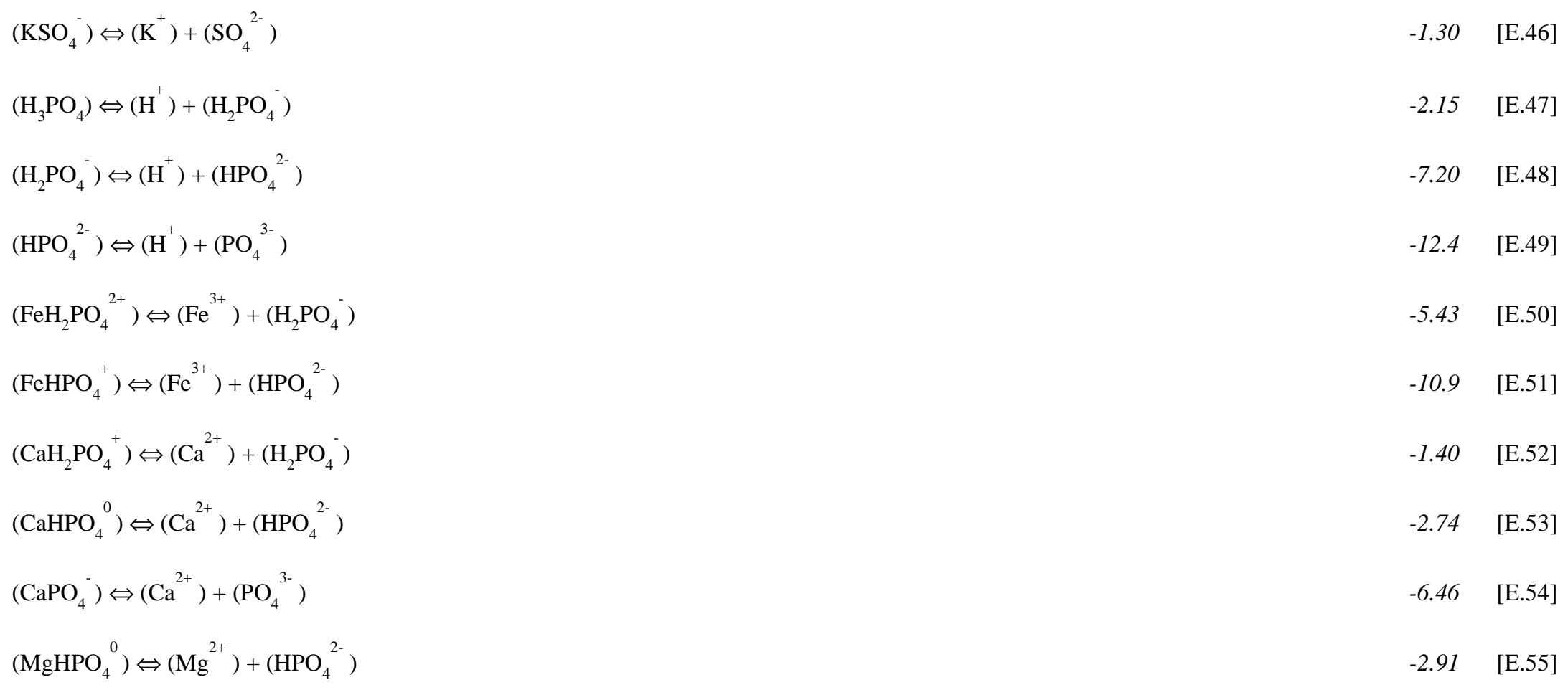

\section{Appendix F: Symbiotic $\mathbf{N}_{\mathbf{2}}$ fixation}

\section{Rhizobial growth}

$R_{\mathrm{max} i, l}=M_{\mathrm{n} i, l} R^{\prime}\left[\chi_{\mathrm{n} i, l}\right] /\left(\left[\chi_{\mathrm{n} i, l}\right]+K_{\chi \mathrm{n}}\right) f_{\mathrm{t}} f_{\mathrm{NP}}$ 


$$
\begin{aligned}
& R_{i, l}=R_{\max i, l}\left(V_{\mathrm{O}_{2}, l, l} / V_{\mathrm{O}_{2} \max i, l}\right) \\
& V_{\mathrm{O}_{2} \max , l}=2.67 R_{\max , l} \\
& V_{\mathrm{O}_{2}, l, l}=V_{\mathrm{O}_{2} \max , l}\left[\mathrm{O}_{2 \mathrm{ri}, l}\right] /\left(\left[\mathrm{O}_{2 \mathrm{r}, i}\right]+K_{\mathrm{O}_{2} \mathrm{r}}\right) \\
& \left.=2 \pi L_{\mathrm{r}, l} D_{\mathrm{sO}_{2}}\left(\left[\mathrm{O}_{2 l l}\right]-\left[\mathrm{O}_{2 \mathrm{ri}, l}\right]\right) / \ln \left(\left(r_{\mathrm{r}, l}+\mathrm{r}_{\mathrm{w} l}\right)\right) / \mathrm{r}_{\mathrm{r}, l}\right) \\
& R_{\mathrm{m} i, l}=\boldsymbol{R}_{\mathrm{m}} N_{\mathrm{ni}, l} f_{\mathrm{tm}} \\
& f_{\mathrm{tm}}=e^{\left[y \left(T l^{-298.16)]}\right.\right.} \\
& R_{\mathrm{g} i, l}=\max \left\{0.0, R_{i, l}-R_{\mathrm{mi}, l}\right\} \\
& R_{\mathrm{si}, l}=\max \left\{0.0, R_{\mathrm{mi}, l}-R_{i, l}\right\} \\
& L_{C i, l}=R_{\mathrm{s} i, l} \min \left\{M_{\mathrm{n} i, l} /\left(2.5 N_{\mathrm{n} i, l}\right), M_{\mathrm{n} i, l} /\left(25.0 P_{\mathrm{n} i, l}\right)\right\}
\end{aligned}
$$

\author{
$\mathrm{O}_{2}$ limitation$$
\mathrm{O}_{2} \text { demand }
$$ \\ equilibrate $\mathrm{O}_{2}$ uptake with

$$
\text { supply }
$$ \\ maintenance respiration \\ temperature function \\ growth + fixation respiration \\ microbial senescence \\ microbial C litterfall
}

product inhibition of $\mathrm{N}_{2}$

$U_{\chi i, l}=\left(R_{\mathrm{g} i, l}-R_{\mathrm{N}_{2} i, l}\right) /\left(1-Y_{\mathrm{n}}^{\prime}\right)$

$\delta M_{\mathrm{n} i, l} / \delta t=U_{i, l} Y_{\mathrm{n}}{ }^{\prime}-L_{C i, l}$

$\delta N_{\mathrm{n} i, l} / \delta t=\delta M_{\mathrm{n} i, l} / \delta t \min \left\{v_{\mathrm{n} i, l} l \chi_{\mathrm{ni} i, l},\left[N_{\mathrm{n}}{ }^{\prime}\right]\right\}$ rate of $\mathrm{N}_{2}$ fixation

\section{fixation}

fixation respiration

growth respiration

[F4]

[F6a]

[F6b]

[F7]

[F8]

[F9]

[F10]

[F12]

[F13]

microbial C growth

[F16] microbial N growth $\quad \delta M_{\mathrm{nd} i, l} / \delta t>0$ 


$$
\begin{aligned}
& \delta N_{\mathrm{ni}, l} / \delta t=N_{\mathrm{n} i, l} / M_{\mathrm{n} i, l} \delta M_{\mathrm{ni}, l} / \delta t \\
& \delta P_{\mathrm{n} i, l} / \delta t=\delta M_{\mathrm{ni}, l} / \delta t \min \left\{\pi_{\mathrm{ni}, l} l \chi_{\mathrm{ni}, l},\left[P_{\mathrm{n}}{ }^{\prime}\right]\right\} \\
& \delta P_{\mathrm{n} i, l} / \delta t=P_{\mathrm{ni}, l} / M_{\mathrm{n} i, l} \delta M_{\mathrm{n} i, l} / \delta t \\
& L_{N i, l}=\operatorname{abs}\left(\delta N_{\mathrm{ni}, l} / \delta t\right) \\
& L_{P i, l}=\operatorname{abs}\left(\delta P_{\mathrm{n} i, l} / \delta t\right)
\end{aligned}
$$

$\begin{array}{ccc}\text { microbial N growth } & \delta M_{\mathrm{nd} i, l} / \delta t<0 & {[\mathrm{~F} 17 \mathrm{~b}]} \\ \text { microbial P growth } & \delta M_{\mathrm{ndi}, l} / \delta t>0 & {[\mathrm{~F} 18 \mathrm{a}]} \\ \text { microbial P growth } & \delta M_{\mathrm{nd} i, l} / \delta t<0 & {[\mathrm{~F} 18 \mathrm{~b}]} \\ \text { microbial N litterfall } & \delta N_{\mathrm{nd}, l, l} / \delta t<0 & {[\mathrm{~F} 19]} \\ \text { microbial P litterfall } & \delta P_{\mathrm{nd}, l} / \delta t<0 & {[\mathrm{~F} 20]}\end{array}$

\section{Nodule-root exchange}

$$
\begin{aligned}
& V_{\chi i, l}=\kappa\left(\chi_{\mathrm{ri}, l} M_{\mathrm{ni}, l}-\chi_{\mathrm{n} i, l} M_{\mathrm{r}, l}\right) /\left(M_{\mathrm{n} i, l}+M_{\mathrm{r}, l}\right) \\
& V_{v i, l}=\kappa\left(v_{\mathrm{ri}, l} \chi_{\mathrm{ni}, l}-v_{\mathrm{ni}, l} \chi_{\mathrm{ri}, l}\right) /\left(\chi_{\mathrm{ni}, l}+\chi_{\mathrm{ri}, l}\right) \\
& V_{\pi i, l}=\kappa\left(\pi_{\mathrm{ri}, l} \chi_{\mathrm{n} i, l}-\pi_{\mathrm{n} i, l} \chi_{\mathrm{ri}, l}\right) /\left(\chi_{\mathrm{n} i, l}+\chi_{\mathrm{r} i, l}\right) \\
& \delta \chi_{\mathrm{ni}, l} / \delta t=V_{\chi i, l}-\min \left\{R_{\mathrm{mi}, l}, R_{i, l}\right\}-R_{\mathrm{N}_{2}, l, l}-U_{\chi i, l}+F_{L C l} L_{C i, l} \\
& \delta v_{\mathrm{n} i, l} / \delta t=V_{v i, l}-\delta N_{\mathrm{ni}, l} / \delta t+V_{\mathrm{N}_{2}, l, l}+F_{L N l} L_{N i, l} \\
& \delta \pi_{\mathrm{ni}, l} / \delta t=V_{\pi i, l}-\delta P_{\mathrm{ni}, l} / \delta t+F_{L P l} L_{P i, l}
\end{aligned}
$$

\section{Definition of variables in appendix $F$}

\begin{tabular}{lllll}
\hline Variable & Definition & Units & Equations & Input Values \\
\hline$B$ & parameter such that $f_{\mathrm{t}}=1.0$ at $T_{l}=298.15 \mathrm{~K}$ & Feference & 17.533
\end{tabular}




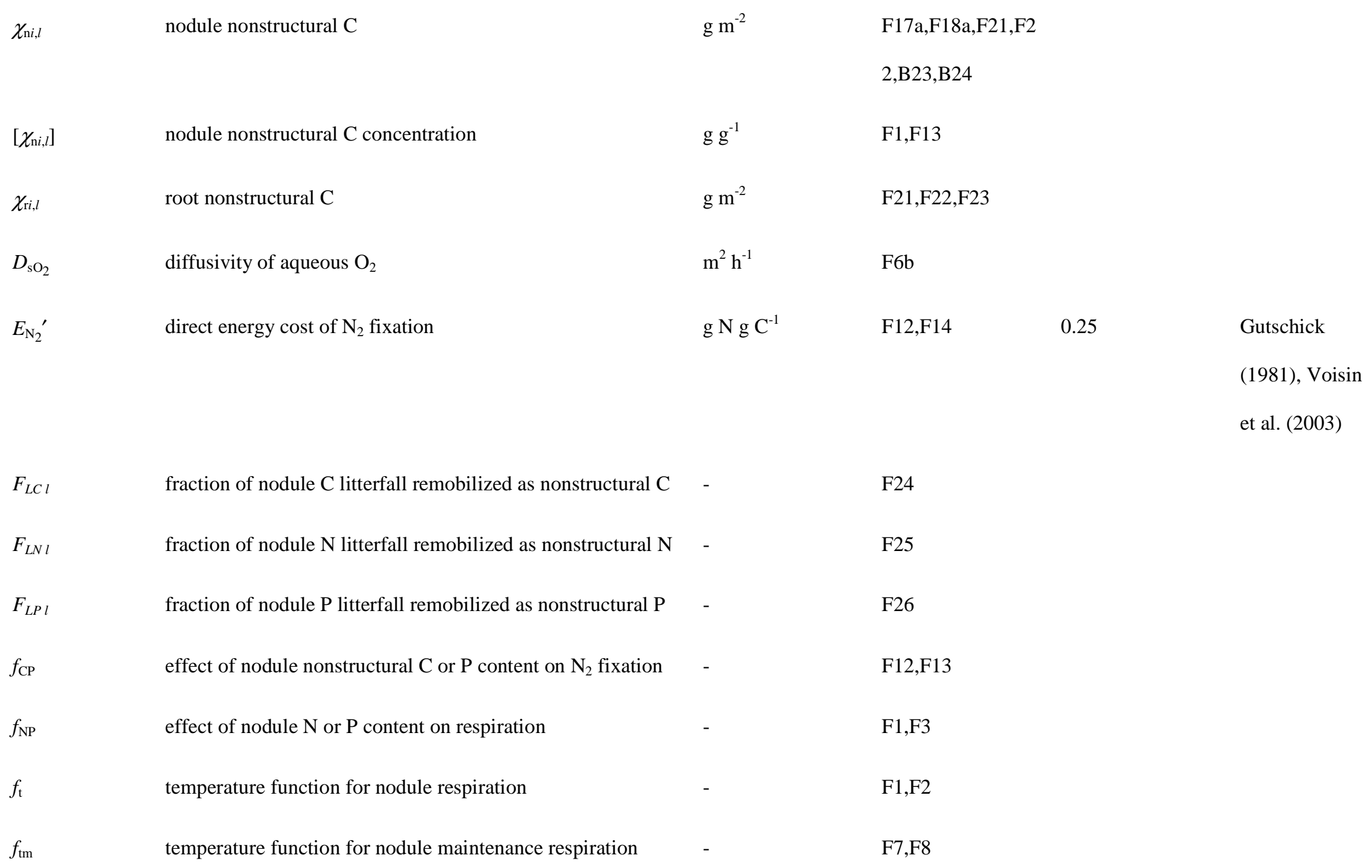




\begin{tabular}{|c|c|c|c|c|}
\hline$H_{\mathrm{a}}$ & energy of activation & $\mathrm{J} \mathrm{mol}^{-1}$ & $\mathrm{~F} 2$ & $57.5 \times 10^{3}$ \\
\hline$H_{\mathrm{dh}}$ & energy of high temperature deactivation & $\mathrm{J} \mathrm{mol}^{-1}$ & F2 & $220 \times 10^{3}$ \\
\hline$H_{\mathrm{dl}}$ & energy of low temperature deactivation & $\mathrm{J} \mathrm{mol}^{-1}$ & $\mathrm{~F} 2$ & $190 \times 10^{3}$ \\
\hline$K_{\not \mathrm{n}}$ & Michaelis-Menten constant for nodule respiration of $\chi_{\mathrm{nd} i l}$ & $\mathrm{~g} \mathrm{~g}^{-1}$ & $\mathrm{~F} 1$ & 0.01 \\
\hline$K_{\mathrm{I} \chi_{\mathrm{n}}}$ & inhibition constant for nonstructural $\mathrm{N}: \mathrm{C}$ on $\mathrm{N}_{2}$ fixation & $\mathrm{g} \mathrm{g}^{-1}$ & F13 & 10 \\
\hline$K_{\mathrm{I} \pi_{\mathrm{n}}}$ & inhibition constant for nonstructural $\mathrm{N}: \mathrm{P}$ on $\mathrm{N}_{2}$ fixation & $\mathrm{g} \mathrm{g}^{-1}$ & F13 & 1000 \\
\hline$K_{\mathrm{N}_{2} \mathrm{r}}$ & Michaelis-Menten constant for nodule $\mathrm{N}_{2}$ uptake & $\mathrm{g} \mathrm{N} \mathrm{m}^{-3}$ & F12 & 0.14 \\
\hline$K_{\mathrm{O} \mathrm{r}}$ & Michaelis-Menten constant for nodule $\mathrm{O}_{2}$ uptake & $\mathrm{g} \mathrm{O} \mathrm{m}^{-3}$ & F6a & \\
\hline$\kappa$ & $\begin{array}{l}\text { rate constant for nonstructural C,N,P exchange between root } \\
\text { and nodule }\end{array}$ & $\mathrm{h}^{-1}$ & $\mathrm{~F} 21, \mathrm{~F} 22, \mathrm{~F} 23$ & \\
\hline$L_{\mathrm{r}, l}$ & root length & $\mathrm{m} \mathrm{m}^{-2}$ & F6b & \\
\hline$L_{C i, l}$ & nodule $\mathrm{C}$ litterfall & $\mathrm{g} \mathrm{C} \mathrm{m}^{-2} \mathrm{~h}^{-1}$ & $\mathrm{~F} 11, \mathrm{~F} 16, \mathrm{~F} 24$ & \\
\hline$L_{N i, l}$ & nodule $\mathrm{N}$ litterfall & $\mathrm{g} \mathrm{N} \mathrm{m}^{-2} \mathrm{~h}^{-1}$ & $\mathrm{~F} 19, \mathrm{~F} 25$ & \\
\hline$L_{P i, l}$ & nodule P litterfall & $\mathrm{gP} \mathrm{m}^{-2} \mathrm{~h}^{-1}$ & $\mathrm{~F} 20, \mathrm{~F} 26$ & \\
\hline
\end{tabular}




\begin{tabular}{|c|c|c|c|c|}
\hline$M_{\mathrm{ni}, l}$ & nodule structural $\mathrm{C}$ & $\mathrm{g} \mathrm{C} \mathrm{m}^{-2}$ & $\mathrm{~F} 1, \mathrm{~F} 11, \mathrm{~F} 12, \mathrm{~F} 16$ & \\
\hline & & & ,F17,F18,F21 & \\
\hline$M_{\mathrm{ri}, l}$ & root structural $\mathrm{C}$ & $\mathrm{g} \mathrm{C} \mathrm{m}^{-2}$ & F21 & \\
\hline$\left[N_{\mathrm{n}}{ }^{\prime}\right]$ & maximum nodule structural $\mathrm{N}$ concentration & $\mathrm{g} \mathrm{N} \mathrm{g} \mathrm{C}^{-1}$ & $\mathrm{~F} 3, \mathrm{~F} 12$ & 0.1 \\
\hline$N_{\mathrm{n} i, l}$ & nodule structural $\mathrm{N}$ & $\mathrm{g} \mathrm{N} \mathrm{m}^{-2}$ & $\mathrm{~F} 7, \mathrm{~F} 11, \mathrm{~F} 12, \mathrm{~F} 17, \mathrm{~F}$ & \\
\hline & & & $19, \mathrm{~F} 25$ & \\
\hline$\left[N_{\mathrm{n} i, l}\right]$ & nodule structural $\mathrm{N}$ concentration & $\operatorname{g~N~g~C}^{-1}$ & $\mathrm{~F} 3, \mathrm{~F} 17 \mathrm{a}$ & \\
\hline$\left[\mathrm{N}_{2 \mathrm{ri}, l}\right]$ & rhizosphere aqueous $\mathrm{N}_{2}$ concentration & $\mathrm{g} \mathrm{N} \mathrm{m}^{-3}$ & F12 & \\
\hline$v_{\mathrm{n}, l}$ & nodule nonstructural $\mathrm{N}$ & $\mathrm{g} \mathrm{N} \mathrm{m}^{-2}$ & $\mathrm{~F} 17 \mathrm{a}, \mathrm{F} 22, \mathrm{~F} 25$ & \\
\hline$v_{\mathrm{r}, l}, l$ & root nonstructural $\mathrm{N}$ & $\mathrm{g} \mathrm{N} \mathrm{m}^{-2}$ & F22 & \\
\hline$\left[v_{\mathrm{n}, i}\right]$ & nodule concentration of nonstructural $\mathrm{N}$ & $\mathrm{g} \mathrm{g}^{-1}$ & $\mathrm{~F} 13, \mathrm{~F} 17 \mathrm{a}$ & \\
\hline$\left[\mathrm{O}_{2 \mathrm{r}, l}\right]$ & rhizosphere aqueous $\mathrm{O}_{2}$ concentration & $\mathrm{g} \mathrm{O} \mathrm{m}^{-3}$ & F6a,b & \\
\hline$\left[\mathrm{O}_{2 l}\right]$ & soil aqueous $\mathrm{O}_{2}$ concentration & $\mathrm{g} \mathrm{O} \mathrm{m}^{-3}$ & F6b & \\
\hline$\left[P_{\mathrm{n}}^{\prime}\right]$ & maximum nodule structural $\mathrm{P}$ concentration & $\mathrm{g} \mathrm{P} \mathrm{g} \mathrm{C}^{-1}$ & F3,F18a & 0.01 \\
\hline
\end{tabular}




\begin{tabular}{|c|c|c|c|c|}
\hline$P_{\mathrm{n} i, l}$ & nodule structural $P$ & $\mathrm{~g} \mathrm{P} \mathrm{m}^{-2}$ & $\mathrm{~F} 18 \mathrm{a}, \mathrm{F} 20, \mathrm{~F} 26$ & \\
\hline$\left[P_{\mathrm{n} i, l}\right]$ & nodule structural $\mathrm{P}$ concentration & $\mathrm{gP} \mathrm{g} \mathrm{C}^{-1}$ & $\mathrm{~F} 3, \mathrm{~F} 11$ & \\
\hline$\pi_{\mathrm{n} i, l}$ & nodule nonstructural $\mathrm{P}$ & $\mathrm{g} \mathrm{P} \mathrm{m}^{-2}$ & $\mathrm{~F} 18 \mathrm{a}, \mathrm{F} 23, \mathrm{~F} 26$ & \\
\hline$\pi_{\mathrm{r}, l}$ & root nonstructural P & $\mathrm{g} \mathrm{P} \mathrm{m}^{-2}$ & F23 & \\
\hline$\left[\pi_{n, l}\right]$ & nodule concentration of nonstructural $\mathrm{P}$ & $\mathrm{g} \mathrm{g}^{-1}$ & F13 & \\
\hline$R$ & gas constant & $\mathrm{J} \mathrm{mol}^{-1} \mathrm{~K}^{-1}$ & F2 & 8.3143 \\
\hline$R_{\mathrm{g} i, l}$ & nodule growth respiration & $\mathrm{g} \mathrm{C} \mathrm{m}^{-2} \mathrm{~h}^{-1}$ & $\mathrm{~F} 9, \mathrm{~F} 12, \mathrm{~F} 15$ & \\
\hline$R^{\prime}$ & $\begin{array}{l}\text { specific nodule respiration at } 25^{\circ} \mathrm{C} \text {, and non-limiting } \mathrm{O}_{2} \text {, } \\
\chi_{\mathrm{nd} i, l}, \nu_{\mathrm{nd} i, l} \text { and } \pi_{\mathrm{nd}, l}\end{array}$ & $\mathrm{~h}^{-1}$ & $\mathrm{~F} 1$ & 0.125 \\
\hline$R_{i, l}$ & nodule respiration under ambient $\mathrm{O}_{2}$ & $\mathrm{gC} \mathrm{m}^{-2} \mathrm{~h}^{-1}$ & $\mathrm{~F} 4, \mathrm{~F} 9, \mathrm{~F} 10, \mathrm{~F} 24$ & \\
\hline $\boldsymbol{R}_{\mathrm{m}}$ & specific nodule maintenance respiration at $25^{\circ} \mathrm{C}$ & $\mathrm{g} \mathrm{Cg} \mathrm{C}^{-1} \mathrm{~h}^{-1}$ & F7 & \\
\hline$R_{\max , l}$ & nodule respiration under non-limiting $\mathrm{O}_{2}$ & $\mathrm{~g} \mathrm{C} \mathrm{m}^{-2} \mathrm{~h}^{-1}$ & $\mathrm{~F} 1, \mathrm{~F} 4, \mathrm{~F} 5$ & \\
\hline$R_{\mathrm{m} i l}$ & nodule maintenance respiration & $\mathrm{g} \mathrm{C} \mathrm{m}^{-2} \mathrm{~h}^{-1}$ & $\mathrm{~F} 7, \mathrm{~F} 9, \mathrm{~F} 10, \mathrm{~F} 24$ & \\
\hline$R_{\mathrm{N}_{2} i, l}$ & nodule respiration for $\mathrm{N}_{2}$ fixation & $\mathrm{g} \mathrm{C} \mathrm{m}^{-2} \mathrm{~h}^{-1}$ & $\mathrm{~F} 14, \mathrm{~F} 15, \mathrm{~F} 24$ & \\
\hline
\end{tabular}




\begin{tabular}{|c|c|c|c|c|}
\hline$R_{\mathrm{s}, l}$ & nodule senescence respiration & $\mathrm{g} \mathrm{C} \mathrm{m}^{-2} \mathrm{~h}^{-1}$ & $\mathrm{~F} 9, \mathrm{~F} 11$ & \\
\hline$r_{\mathrm{r}, l}$ & root radius & $\mathrm{m}$ & F6b & \\
\hline $\mathrm{r}_{\mathrm{w} l}$ & radius of soil water films & $\mathrm{m}$ & F6b & \\
\hline$S$ & change in entropy & $\mathrm{J} \mathrm{mol}^{-1} \mathrm{~K}^{-1}$ & $\mathrm{~F} 2$ & 710 \\
\hline$T_{l}$ & soil temperature & K & $\mathrm{F} 2, \mathrm{~F} 8$ & \\
\hline$U_{\chi i, l}$ & uptake of nodule nonstructural $\mathrm{C}$ for growth & $\mathrm{gC} \mathrm{m}^{-2} \mathrm{~h}^{-1}$ & $\mathrm{~F} 15, \mathrm{~F} 16, \mathrm{~F} 24$ & \\
\hline$V_{\chi i, l}$ & nonstructural $\mathrm{C}$ transfer between root and nodule & $\mathrm{g} \mathrm{C} \mathrm{m}^{-2} \mathrm{~h}^{-1}$ & $\mathrm{~F} 21, \mathrm{~F} 24$ & \\
\hline$V_{v i, l}$ & nonstructural $\mathrm{N}$ transfer between root and nodule & $\mathrm{g} \mathrm{N} \mathrm{m}^{-2} \mathrm{~h}^{-1}$ & $\mathrm{~F} 22, \mathrm{~F} 25$ & \\
\hline$V_{\mathrm{N}_{2}, i}$ & $\mathrm{~N}_{2}$ fixation & $\mathrm{g} \mathrm{N} \mathrm{m}^{-2} \mathrm{~h}^{-1}$ & $\mathrm{~F} 12, \mathrm{~F} 14, \mathrm{~F} 25$ & \\
\hline$V_{\mathrm{O}_{2} \max i, l}$ & $\mathrm{O}_{2}$ uptake by nodules under non-limiting $\mathrm{O}_{2}$ & $\mathrm{gO} \mathrm{m}^{-2} \mathrm{~h}^{-1}$ & F4,F5,F6a & \\
\hline$V_{\mathrm{O} 2 i, l}$ & $\mathrm{O}_{2}$ uptake by nodules under ambient $\mathrm{O}_{2}$ & $\mathrm{~g} \mathrm{O} \mathrm{m}^{-2} \mathrm{~h}^{-1}$ & $\mathrm{~F} 4, \mathrm{~F} 6$ & \\
\hline$V_{\pi, l}$ & nonstructural $\mathrm{P}$ transfer between root and nodule & $\mathrm{gP} \mathrm{m}^{-2} \mathrm{~h}^{-1}$ & $\mathrm{~F} 23, \mathrm{~F} 26$ & \\
\hline$Y_{\mathrm{n}}^{\prime}$ & nodule growth yield & $\mathrm{g} \mathrm{C} \mathrm{g} \mathrm{C}^{-1}$ & F15,F16 & 0.67 \\
\hline y & shape parameter for $f_{\mathrm{tm}}$ & - & F8 & 0.081 \\
\hline
\end{tabular}




\section{Appendix G: $\mathrm{CH}_{4}$ production and consumption}

\section{Anaerobic fermenters and $\mathrm{H}_{2}$ producing acetogens}

$\mathrm{R} i, f=\left\{\boldsymbol{R}^{\prime} f \mathrm{Mi}, f, a[\mathrm{DOC} i, c] /\left(\mathbf{K} \boldsymbol{f}\left(\left(1+\left[\mathrm{O}_{2}\right] / \mathbf{K}_{i}\right)+[\mathrm{DOC} i, c]\right)\right\} \mathrm{ft}\right.$

DOCi, $c \rightarrow 0.67 \mathrm{~A}_{i, c}+0.33 \mathrm{CO}_{2}-\mathrm{C}+0.11 \mathrm{H}_{2}$

$\mathrm{U} i, f, c=\mathrm{Rm} i, f+(\mathrm{R} i, f-\mathrm{Rm} i, f)(1.0+\mathrm{Y} f)$

$\mathrm{U} i, f, c=\mathrm{R} i, f$

$\mathrm{Y} f=-\Delta \mathrm{G} f / \mathbf{E}_{\mathbf{M}}$

$\Delta \mathrm{G} f=\Delta \mathbf{G}^{\prime} f+\left\{\boldsymbol{R} \operatorname{Tln}\left(\left[\mathrm{H}_{2}\right] /\left[\mathbf{H}_{2}{ }^{\prime}\right]\right)^{4}\right\}$

$\delta \mathrm{M} i, f, j, c / \delta \mathrm{t}=\mathrm{F} j \mathrm{U} i, f, c-\mathrm{F} j \mathrm{R} i, f-\mathrm{D} i, f, j, c$

$\delta \mathrm{M} i, f, j, c / \delta \mathrm{t}=\mathrm{F} j \mathrm{U} i, f, c-\mathrm{Rm} i, f, j-\mathrm{D} i, f, j, c$

\section{Acetotrophic methanogens}

$\mathrm{R}_{i, m}=\left\{\boldsymbol{R}_{\boldsymbol{m}}^{\prime} \mathrm{M}_{i, m, a}\left[\mathrm{~A}_{i, c}\right] /\left(\mathbf{K}_{\boldsymbol{m}}+\left[\mathrm{A}_{i, c}\right]\right)\right\} \mathrm{f}_{\mathrm{t}}$

$\mathrm{A} i, c \rightarrow 0.50 \mathrm{CH}_{4}-\mathrm{C}+0.50 \mathrm{CO}_{2}-\mathrm{C}$

$\mathrm{U}_{i, m, c}=\mathrm{R}_{\mathrm{m} i, m}+\left(\mathrm{R}_{i, m}-\mathrm{R}_{\mathrm{m} i, m}\right)\left(1.0+\mathrm{Y}_{m}\right)$ respiration by fermenters

[G1]

partition respiration products

uptake by fermenters

$[\mathrm{Ri} f>\mathrm{Rm} i, f] \quad[\mathrm{G} 3 \mathrm{a}]$

$[\mathrm{Ri} f<\mathrm{Rmi}, f] \quad[\mathrm{G} 3 \mathrm{~b}$

growth yield of fermentation

free energy change of fermentation

growth of fermenters

$[\mathrm{Ri}, f>\mathrm{Rm} i, f]$

$[\mathrm{R} i, f<\mathrm{Rmi} f]$

respiration by acetotrophic

methanogens

partition respiration products

uptake by acetotrophic

$\left[\mathrm{R}_{i, m}>\mathrm{R}_{\mathrm{m} i, m}\right]$

[G9a] 
$\mathrm{U}_{i, m, c}=\mathrm{R}_{i, m}$

$-\mathrm{Y}_{m}=-\Delta \mathbf{G}_{\boldsymbol{m}}^{\prime} / \mathbf{E}_{\mathrm{M}}$

$\delta \mathrm{M}_{i, m, j, c} / \delta \mathrm{t}=\mathrm{F}_{j} \mathrm{U}_{i, m, c}-\mathrm{F}_{j} \mathrm{R}_{i, m}-\mathrm{D}_{i, m, j, c}$

$\delta \mathrm{M}_{i, m, j, c} / \delta \mathrm{t}=\mathrm{F}_{j} \mathrm{U}_{i, m, c}-\mathrm{R}_{\mathrm{m} i, m, j}-\mathrm{D}_{i, m, j, c}$

\section{Hydrogenotrophic methanogens}

$\mathrm{R}_{h}=\left\{\boldsymbol{R}_{\boldsymbol{h}}^{\prime} \mathrm{M}_{h, a}\left[\mathrm{H}_{2}\right] /\left(\mathbf{K}_{\boldsymbol{h}}+\left[\mathrm{H}_{2}\right]\right)\left[\mathrm{CO}_{2}\right] /\left(\mathbf{K}_{\boldsymbol{c}}+\left[\mathrm{CO}_{2}\right]\right)\right\} \mathrm{f}_{\mathrm{t}}$

$\mathrm{CO}_{2}-\mathrm{C}+0.67 \mathrm{H}_{2} \rightarrow \mathrm{CH}_{4}-\mathrm{C}+3 \mathrm{H}_{2} \mathrm{O}$

$\mathrm{U}_{h, c}=\mathrm{R}_{\mathrm{m} h}+\left(\mathrm{R}_{h}-\mathrm{R}_{\mathrm{m} h}\right)\left(1.0+\mathrm{Y}_{h}\right)$

$\mathrm{U}_{h, c}=\mathrm{R}_{h}$

$\mathrm{Y}_{h}=-\Delta \mathrm{G}_{h} / \mathbf{E}_{\mathbf{C}}$

$\Delta \mathrm{G}_{h}=\boldsymbol{\Delta} \mathbf{G}_{\boldsymbol{h}}^{\prime} \boldsymbol{}-\left\{\boldsymbol{R} \operatorname{Tln}\left(\left[\mathrm{H}_{2}\right] /\left[\mathbf{H}_{2}{ }^{\prime}\right]\right)^{4}\right\}$
$\left[\mathrm{R}_{i, m}<\mathrm{R}_{\mathrm{m} i, m}\right]$

growth yield of acetotrophic

[G10]

methanogenesis

growth of acetotrophic

$\left[\mathrm{R}_{i, m}>\mathrm{R}_{\mathrm{m} i, m}\right]$

[G11a]

methanogens

$\left[\mathrm{R}_{i, m}<\mathrm{R}_{\mathrm{m} i, m}\right]$

$[\mathrm{G} 11 \mathrm{~b}$

respiration by hydrogenotrophic

methanogens

partition respiration products

uptake by hydrogenotrophic

$\left[\mathrm{R}_{h}>\mathrm{R}_{\mathrm{m} h}\right]$

[G14a]

methanogens

$\left[\mathrm{R}_{h}<\mathrm{R}_{\mathrm{m} h}\right] \quad[\mathrm{G} 14 \mathrm{~b}]$

growth yield of hydrogenotrophic

methanogenesis

free energy change of

hydrogenotrophic methanogenesis 
$\delta \mathrm{M}_{h, j, c} / \delta \mathrm{t}=\mathrm{F}_{j} \mathrm{U}_{h, c}-\mathrm{F}_{j} \mathrm{R}_{h}-\mathrm{D}_{h, j, c}$

$\delta \mathrm{M}_{h, j, c} / \delta \mathrm{t}=\mathrm{F}_{j} \mathrm{U}_{h, c}-\mathrm{R}_{\mathrm{m} h, j}-\mathrm{D}_{h, j, c}$

\section{Autotrophic methanotrophs}

$\mathrm{X}_{t}^{\prime}=\left\{\boldsymbol{X}_{\boldsymbol{t}}^{\prime} \mathrm{M}_{t, a}\left[\mathrm{CH}_{4}\right] /\left(\mathbf{K}_{\boldsymbol{t}}+\left[\mathrm{CH}_{4}\right]\right)\right\} f_{\mathrm{t}}$

$\mathrm{R}_{t}^{\prime}=\mathrm{X}_{t}^{\prime} \mathrm{Y}_{t_{\mathrm{R}}}$

$\mathrm{Y}_{t_{\mathrm{R}}}=-\Delta \mathbf{G}_{\boldsymbol{t}}^{\prime} / \mathbf{E}_{\mathrm{G}}$

$\mathrm{X}_{t}=\mathrm{X}_{t}^{\prime} f_{\mathrm{O}_{2} t}$

$\mathrm{R}_{t}=\mathrm{R}_{t}^{\prime} f_{\mathrm{O}_{2} t}$

$\mathrm{CH}_{4}-\mathrm{C}+4.0 \mathrm{O}_{2} \rightarrow \mathrm{CO}_{2}-\mathrm{C}+1.5 \mathrm{H}_{2} \mathrm{O}+0.167 \mathrm{H}^{+}$

$\mathrm{CH}_{4}-\mathrm{C}+1.33 \mathrm{O}_{2} \rightarrow \mathrm{CH}_{2} \mathrm{O}-\mathrm{C}+0.167 \mathrm{H}^{+}$ growth of hydrogenotrophic

$\left[\mathrm{R}_{h}>\mathrm{R}_{\mathrm{m} h}\right]$

[G17a]

methanogens

$\left[\mathrm{R}_{h}<\mathrm{R}_{\mathrm{m} h}\right]$

$[\mathrm{G} 17 \mathrm{~b}]$

$\mathrm{CH}_{4}$ oxidation by methanotrophs

under non-limiting $\mathrm{O}_{2}$

respiration by methanotrophs under

non-limiting $\mathrm{O}_{2}$

energy yield from $\mathrm{CH}_{4}$ oxidation

$\mathrm{CH}_{4}$ oxidation by methanotrophs

under ambient $\mathrm{O}_{2}$

respiration by methanotrophs under

ambient $\mathrm{O}_{2}$

$\mathrm{O}_{2}$ requirements for $\mathrm{CH}_{4}$ oxidation

by methanotrophs

$\mathrm{O}_{2}$ requirements for growth by

methanotrophs 
$\mathrm{CH}_{2} \mathrm{O}-\mathrm{C}+2.67 \mathrm{O}_{2} \rightarrow \mathrm{CO}_{2}-\mathrm{C}+1.5 \mathrm{H}_{2} \mathrm{O}$

$\mathrm{U}_{t, c}=\mathrm{R}_{\mathrm{m} t}+\left(\mathrm{R}_{t}-\mathrm{R}_{\mathrm{m} t}\right)\left(1.0+\mathrm{Y}_{t_{\mathrm{G}}}\right)$

$\mathrm{U}_{t, c}=\mathrm{R}_{t}$

$\mathrm{Y}_{t_{\mathrm{G}}}=-\Delta \mathbf{G}^{\prime}{ }_{c} / \mathbf{E}_{\mathrm{M}}$

$\delta \mathrm{M}_{t, j, c} / \delta \mathrm{t}=\mathrm{F}_{j} \mathrm{U}_{t, c}-\mathrm{F}_{j} \mathrm{R}_{t}-\mathrm{D}_{t, j, c}$

$\delta \mathrm{M}_{t, j, c} / \delta \mathrm{t}=\mathrm{F}_{j} \mathrm{U}_{t, c}-\mathrm{R}_{\mathrm{m} t, j}-\mathrm{D}_{t, j, c}$
$\mathrm{O}_{2}$ requirements for respiration by

[G24]

methanotrophs

uptake by methanotrophs

$\left[\mathrm{R}_{t}>\mathrm{R}_{\mathrm{m} t}\right]$

[G25a]

$\left[\mathrm{R}_{t}<\mathrm{R}_{\mathrm{m} t}\right]$

[G25b]

growth yield of methanotrophy

[G26]

$\left[\mathrm{R}_{t}>\mathrm{R}_{\mathrm{m} t}\right] \quad[\mathrm{G} 27 \mathrm{a}]$

$\left[\mathrm{R}_{t}<\mathrm{R}_{\mathrm{m} t}\right] \quad[\mathrm{G} 27 \mathrm{~b}]$

Definition of variables in appendix G

\begin{tabular}{|c|c|c|c|c|c|}
\hline Variable & Definition & Units & Equations & Input Values & Reference \\
\hline A & acetate & $\mathrm{g} \mathrm{C} \mathrm{m}^{-2}$ & {$[\mathrm{G} 2]$} & & \\
\hline$[\mathrm{A}]$ & aqueous concentration of acetate & $\mathrm{g} \mathrm{C} \mathrm{m}^{-3}$ & {$[\mathrm{G} 7]$} & & \\
\hline$a$ & descriptor for $j=$ active component of $\mathrm{M} i$ & & & & \\
\hline$\left[\mathrm{CH}_{4}\right]$ & aqueous concentration of $\mathrm{CH}_{4}$ & $\mathrm{~g} \mathrm{C} \mathrm{m}^{-3}$ & {$[\mathrm{G} 18]$} & & \\
\hline$\left[\mathrm{CO}_{2}\right]$ & aqueous concentration of $\mathrm{CO}_{2}$ & $\mathrm{~g} \mathrm{C} \mathrm{m}^{-3}$ & {$[\mathrm{G} 12]$} & & \\
\hline $\mathrm{D} h, j, c$ & decomposition of hydrogenotrophic methanogens & $\mathrm{g} \mathrm{C} \mathrm{m}^{-2} \mathrm{~h}^{-1}$ & {$[\mathrm{G} 17]$} & & \\
\hline
\end{tabular}




\begin{tabular}{|c|c|c|c|c|c|}
\hline $\mathrm{D} i, f, j, c$ & decomposition of fermenters and acetogens & $\mathrm{gC} \mathrm{m}^{-2} \mathrm{~h}^{-1}$ & [G6] & & \\
\hline $\mathrm{D} i, m, j, c$ & decomposition of acetotrophic methanogens & $\mathrm{g} \mathrm{C} \mathrm{m}^{-2} \mathrm{~h}^{-1}$ & {$[\mathrm{G} 11]$} & & \\
\hline $\mathrm{D} t, j, c$ & decomposition of autotrophic methanotrophs & $\mathrm{g} \mathrm{C} \mathrm{m}^{-2} \mathrm{~h}^{-1}$ & [G27] & & \\
\hline $\mathbf{E}_{\mathbf{C}}$ & energy required to construct new $\mathrm{M}$ from $\mathrm{CO}_{2}$ & $\mathrm{~kJ} \mathrm{~g} \mathrm{C}^{-1}$ & [G15] & 75 & \\
\hline $\mathbf{E}_{\mathbf{G}}$ & energy required to transform $\mathrm{CH}_{4}$ into organic $\mathrm{C}$ & $\mathrm{kJ} \mathrm{g} \mathrm{C}^{-1}$ & [G20] & 23.5 & $\begin{array}{l}\text { Anthony } \\
\text { (1982) }\end{array}$ \\
\hline $\mathbf{E}_{\mathbf{M}}$ & energy required to construct new $\mathrm{M}$ from organic $\mathrm{C}$ & $\mathrm{kJ} \mathrm{g} \mathrm{C}^{-1}$ & {$[\mathrm{G} 4, \mathrm{G} 10, \mathrm{G} 26]$} & 25 & \\
\hline $\mathrm{Fj}$ & partitioning coefficient for $j$ in $\mathrm{M} i, n, j$ & & $\begin{array}{l}{[\mathrm{G} 6, \mathrm{G} 11, \mathrm{G} 17, \mathrm{G} 2} \\
7]\end{array}$ & & \\
\hline$f$ & descriptor for fermenters and acetogens in each $\mathrm{M}_{i}$ & & & & \\
\hline$f_{2} t$ & ratio of $\mathrm{O}_{2}$ uptake to $\mathrm{O}_{2}$ requirement for $\mathrm{CH}_{4}$ oxidation & & {$[\mathrm{G} 21 \mathrm{a}, \mathrm{b}]$} & & \\
\hline$f_{t}$ & $\begin{array}{l}\text { temperature function for growth-related processes } \\
\text { (dimensionless) }\end{array}$ & & {$[\mathrm{G} 1, \mathrm{G} 7, \mathrm{G} 12]$} & & \\
\hline$\Delta \mathbf{G}_{c}^{\prime}$ & free energy change of $\mathrm{C}$ oxidation- $\mathrm{O}_{2}$ reduction & $\mathrm{kJ} \mathrm{g} \mathrm{C}^{-1}$ & [G26] & -37.5 & Brock and \\
\hline & & & & & $\begin{array}{l}\text { Madigan } \\
\text { (1991) }\end{array}$ \\
\hline
\end{tabular}


Brock and

Madigan

(1991),

Schink (1997)

$\Delta \mathrm{G}_{h}$

Brock and

Madigan

(1991)

Brock and

Madigan

(1991),

Schink (1997)

$\Delta \mathbf{G}^{\prime}$

Brock and 
$\left[\mathrm{H}_{2}\right]$

$\left[\mathbf{H}_{2}{ }^{\prime}\right]$

aqueous concentration of $\mathrm{H}_{2}$

aqueous concentration of $\mathrm{H}_{2}$ when $\Delta \mathrm{G}_{h}=\Delta \mathrm{G}_{h}^{\prime}$ and $\Delta \mathrm{G}_{f}=$

$\Delta \mathrm{G}_{f}^{\prime}$

descriptor for hydrogenotrophic methanogens in each $\mathrm{M}_{i}$

descriptor for organic matter-microbe complex $(i=$ plant

residue, manure, particulate $\mathrm{OM}$, or humus)

descriptor for structural or kinetic components for each

functional type within each $\mathrm{M}_{i}($ e.g. $a=$ active)

M-M constant for uptake of $\mathrm{CO}_{2}$ by hydrogenotrophic

methanogens

M-M constant for uptake of $\mathrm{DOC}_{i, c}$ by fermenters and

acetogens

inhibition constant for $\mathrm{O}_{2}$ on fermentation

M-M constant for uptake of $\mathrm{H}_{2}$ by hydrogenotrophic

methanogens $\mathrm{g} \mathrm{H} \mathrm{m}^{-3}$

$\mathrm{g} \mathrm{H} \mathrm{m}^{-3}$

$150 \times 10^{-6}$

Brock and

Madigan

(1991)

$\mathrm{g} \mathrm{C} \mathrm{m}^{-3} \quad[\mathrm{G} 12]$

0.12

$\mathrm{g} \mathrm{C} \mathrm{m}^{-3}$

[G1]

12

McGill et al.

(1981)

$\mathrm{g} \mathrm{O} \mathrm{m}^{-3}$

[G1]

0.064

$\mathrm{g} \mathrm{H} \mathrm{m}^{-3}$
Robinson and

Tiedje (1982), 
methanogens

Zehnder et al.

(1980)

$\mathbf{K}_{t}$

M-M constant for uptake of $\mathrm{CH}_{4}$ by methanotrophs

$\mathrm{g} \mathrm{C} \mathrm{m}^{-3}$

[G18]

descriptor for elemental fraction within each $j(j=c, n$ or $p)$ hydrogenotrophic methanogen community

fermenter and acetogenic community

acetotrophic methanogen community

autotrophic methanotrophic community

descriptor for acetotrophic methanogens in each $\mathrm{M}_{i}$

$\mathrm{g} \mathrm{C} \mathrm{m}^{-2}$

$\mathrm{g} \mathrm{C} \mathrm{m}^{-2}$

[G12,G17]

$\mathrm{g} \mathrm{C} \mathrm{m}^{-2}$

$[\mathrm{G} 1, \mathrm{G} 6]$

$\mathrm{g} \mathrm{C} \mathrm{m}^{-2}$

$[\mathrm{G} 7, \mathrm{G} 11]$

$\mathrm{g} \mathrm{C} \mathrm{m}^{-2}$

[G18,G27]

DOC

[DOC soluble organic matter

aqueous concentration of soluble organic matter

gas constant

descriptor for acetotrophic methanogens in each
soluble organic matter
aqueous concentration of soluble organic matter
gas constant


(1971)

Wofford et al.

(1986)

$\mathrm{R}_{h}$
$\mathrm{CO}_{2}$ reduction by hydrogenotrophic methanogens

specific $\mathrm{CO}_{2}$ reduction by hydrogenotrophic methanogens at

saturating $\left[\mathrm{H}_{2}\right]$ and $\left[\mathrm{CO}_{2}\right]$, and at $30{ }^{\circ} \mathrm{C}$ and zero water

potential

$\mathrm{g} \mathrm{C} \mathrm{m} \mathrm{m}^{-2}$

$\mathrm{g} \mathrm{Cg} \mathrm{M}_{h, a}^{-1} \mathrm{~h}^{-1}$

Shea et al.

(1968),

Zehnder and

Wuhrmann

(1977)

$\mathrm{g} \mathrm{C} \mathrm{m}^{-2} \mathrm{~h}^{-1} \quad[\mathrm{G} 1, \mathrm{G} 2, \mathrm{G} 3, \mathrm{G} 6]$

$\mathrm{g} \mathrm{C} \mathrm{m} \mathrm{m}^{-2} \mathrm{~h}^{-1}$

$[\mathrm{G} 7, \mathrm{G} 8, \mathrm{G} 9, \mathrm{G} 11]$

$\operatorname{g~C~g~M~}{ }_{i, m, a}^{-1} \mathrm{~h}^{-1}$

[G7]

0.20

Smith and

Mah (1980)

saturating $\left[\mathrm{A}_{i, c}\right], 30^{\circ} \mathrm{C}$ and zero water potential

maintenance respiration by hydrogenotrophic methanogens

$\mathrm{g} \mathrm{C} \mathrm{m}^{-2} \mathrm{~h}^{-1}$

[G14,G17] 


\begin{tabular}{|c|c|c|c|}
\hline $\mathrm{R}_{\mathrm{m} i f, j}$ & maintenance respiration by fermenters and acetogens & $\mathrm{gC} \mathrm{m}^{-2} \mathrm{~h}^{-1}$ & {$[\mathrm{G} 3, \mathrm{G} 6]$} \\
\hline $\mathrm{R}_{\mathrm{m} i, m, j}$ & maintenance respiration by acetotrophic methanogens & $\mathrm{g} \mathrm{C} \mathrm{m}^{-2} \mathrm{~h}^{-1}$ & {$[\mathrm{G} 9, \mathrm{G} 11]$} \\
\hline $\mathrm{R}_{\mathrm{m} t, j}$ & maintenance respiration by methanotrophs & $\mathrm{g} \mathrm{C} \mathrm{m}^{-2} \mathrm{~h}^{-1}$ & {$[\mathrm{G} 25, \mathrm{G} 27]$} \\
\hline $\mathrm{R}_{t}$ & $\mathrm{CH}_{4}$ oxidation by methanotrophs for respiration & $\mathrm{gC} \mathrm{m}^{-2} \mathrm{~h}^{-1}$ & {$[\mathrm{G} 21 \mathrm{~b}, \mathrm{G} 23, \mathrm{G} 24$,} \\
\hline & & & $\mathrm{G} 25, \mathrm{G} 27 \mathrm{a}]$ \\
\hline $\mathrm{R}_{t}^{\prime}$ & $\begin{array}{l}\mathrm{CH}_{4} \text { oxidation by methanotrophs for respiration at saturating } \\
\mathrm{O}_{2}\end{array}$ & $\mathrm{~g} \mathrm{C} \mathrm{m}^{-2} \mathrm{~h}^{-1}$ & {$[\mathrm{G} 19, \mathrm{G} 21 \mathrm{~b}]$} \\
\hline $\mathrm{T}$ & soil temperature & $\mathrm{K}$ & {$[\mathrm{G} 5, \mathrm{G} 16]$} \\
\hline$t$ & descriptor for autotrophic methanotrophs & & \\
\hline $\mathrm{U}_{h, c}$ & rate of $\mathrm{CO}_{2}$ uptake by $\mathrm{M}_{h}$ & $\mathrm{~g} \mathrm{C} \mathrm{m}^{-2} \mathrm{~h}^{-1}$ & {$[\mathrm{G} 14, \mathrm{G} 17, \mathrm{G} 18]$} \\
\hline $\mathrm{U}_{i, f, k}$ & rate of $\mathrm{DOC}_{i, k}$ uptake by $\mathrm{M}_{i, f}$ & $\mathrm{gC} \mathrm{m}^{-2} \mathrm{~h}^{-1}$ & {$[\mathrm{G} 3, \mathrm{G} 6]$} \\
\hline $\mathrm{U}_{i, m, c}$ & rate of $\mathrm{A}_{i, c}$ uptake by $\mathrm{M}_{i, m}$ & $\mathrm{gC} \mathrm{m}^{-2} \mathrm{~h}^{-1}$ & {$[\mathrm{G} 9, \mathrm{G} 11]$} \\
\hline $\mathrm{U}_{t, c}$ & rate of $\mathrm{CH}_{4}$ uptake by $\mathrm{M}_{t}$ & $\mathrm{gC} \mathrm{m}^{-2} \mathrm{~h}^{-1}$ & {$[\mathrm{G} 25, \mathrm{G} 27]$} \\
\hline $\mathrm{X}_{t}$ & $\mathrm{CH}_{4}$ oxidation by methanotrophs & $\mathrm{g} \mathrm{C} \mathrm{m}^{-2} \mathrm{~h}^{-1}$ & {$[\mathrm{G} 21 \mathrm{a}, \mathrm{G} 22]$} \\
\hline
\end{tabular}




$\begin{array}{llll}\mathrm{X}_{t}^{\prime} & \mathrm{CH}_{4} \text { oxidation by methanotrophs at saturating } \mathrm{O}_{2} & \mathrm{~g} \mathrm{C} \mathrm{m}^{-2} \mathrm{~h}^{-1} & {[\mathrm{G} 1, \mathrm{G} 2, \mathrm{G} 4 \mathrm{a}]} \\ \boldsymbol{X}_{\boldsymbol{t}}^{\prime} & \text { specific } \mathrm{CH}_{4} \text { oxidation by methanotrophs at saturating } \mathrm{O}_{2}, 30 & \mathrm{~g} \mathrm{C} \mathrm{g}^{-1} \mathrm{~h}^{-1} & {[\mathrm{G} 18]} \\ & { }^{\circ} \mathrm{C} \text { and zero water potential } & & \\ \mathrm{Y}_{f} & \text { biomass yield from fermentation and acetogenic reactions } & \mathrm{g} \mathrm{M}_{i, f} \mathrm{~g} \mathrm{DOC}_{i, c}^{-1} & {[\mathrm{G} 3, \mathrm{G} 4]} \\ \mathrm{Y}_{h} & \text { biomass yield from hydrogenotrophic methanogenic reaction } & \mathrm{g} \mathrm{M}_{h} \mathrm{~g} \mathrm{CO}_{2}-\mathrm{C}^{-1} & {[\mathrm{G} 14, \mathrm{G} 15, \mathrm{G} 18]} \\ \mathrm{Y}_{m} & \text { biomass yield from acetotrophic methanogenic reaction } & \mathrm{g} \mathrm{M}_{i, m} \mathrm{~g} \mathrm{~A}_{i, c}{ }^{-1} & {[\mathrm{G} 9, \mathrm{G} 10]} \\ \mathrm{Y}_{t_{\mathrm{G}}} & \text { biomass yield from methanotrophic growth respiration } & \mathrm{g} \mathrm{M}_{t}-\mathrm{C} \mathrm{g} \mathrm{CH}_{4}-\mathrm{C}^{-1} & {[\mathrm{G} 25 \mathrm{a}, \mathrm{G} 26]} \\ \mathrm{Y}_{t_{\mathrm{R}}} & \text { ratio of } \mathrm{CH}_{4} \text { respired vs. } \mathrm{CH}_{4} \text { oxidized by methanotrophs } & \mathrm{g} \mathrm{C} \mathrm{g} \mathrm{C}^{-1} & {[\mathrm{G} 19, \mathrm{G} 20]}\end{array}$

\section{Appendix H: Inorganic N transformations}

\section{Mineralization and immobilization of $\mathrm{NH}_{4}{ }^{+}$by all microbial populations}

$$
\begin{aligned}
& I_{\mathrm{NH}_{4} i, n, j}=\left(M_{i, m, j, \mathrm{C}} C_{\mathrm{Nj}}-M_{i, m, j, \mathrm{~N}}\right) \\
& I_{\mathrm{NH}_{4} i, n, j}=\left(M_{i, m, j, \mathrm{C}} C_{\mathrm{Nj}}-M_{i, m, j, \mathrm{~N}}\right)\left[\mathrm{NH}_{4}^{+}\right] /\left(\left[\mathrm{NH}_{4}^{+}\right]+\boldsymbol{K}_{\mathrm{NH}_{4} m}\right) \\
& I_{\mathrm{NO}_{3} i, n, j}=\left(M_{i, m, j, \mathrm{C}} C_{\mathrm{N} j}-\left(M_{i, m, j, \mathrm{~N}}+I_{\mathrm{NH}_{4} i, n, j}\right)\right)\left[\mathrm{NO}_{3}^{-}\right] /\left(\left[\mathrm{NO}_{3}^{-}\right]+K_{\mathrm{NO}_{3} m}\right)
\end{aligned}
$$

$$
\begin{array}{lr}
\left(I_{\mathrm{NH}_{4} i, n, j}<0\right) & {[\mathrm{H} 1 \mathrm{a}]} \\
\left(I_{\mathrm{NH}_{4} i, n, j}>0\right) & {[\mathrm{H} 1 \mathrm{~b}]} \\
\left(I_{\mathrm{NO}_{3} i, n, j}>0\right) & {[\mathrm{H} 1 \mathrm{~b}]}
\end{array}
$$

\section{Oxidation of DOC and reduction of $\mathrm{O}_{2}$ by heterotrophs}

$X_{\mathrm{DOC} i, h}^{\prime}=\left\{X_{\mathrm{DOC}}^{\prime} M_{i, h, a}\left[\mathrm{DOC}_{i}\right] /\left(\left[\mathrm{DOC}_{i}\right]\right)+\boldsymbol{K}_{\mathbf{X} h}\right\} f_{\mathrm{t}}$ 
$R_{\mathrm{O}_{2} i, h}^{\prime}=\mathbf{R} \mathbf{Q}_{\mathbf{C}} X_{\mathrm{DOC} i, h}^{\prime}$

$R_{\mathrm{O}_{2} i, h}=4 \pi n M_{i, h, a} D_{\mathrm{sO}_{2}}\left(\left[\mathrm{O}_{2 \mathrm{~s}}\right]-\left[\mathrm{O}_{2 \mathrm{~m} i, h}\right]\right)\left[r_{\mathrm{m}} r_{\mathrm{w}} /\left(r_{\mathrm{w}}-r_{\mathrm{m}}\right)\right]$

$=R_{\mathrm{O}_{2} i, h}^{\prime}\left[\mathrm{O}_{2 \mathrm{~m} i, h}\right] /\left(\left[\mathrm{O}_{2 \mathrm{~m} i, h}\right]+\boldsymbol{K}_{\mathbf{O}_{2} h}\right)$

$X_{\mathrm{DOC} i, h}=X_{\mathrm{DOC} i, h}^{\prime} R_{\mathrm{O}_{2} i, h} / R_{\mathrm{O}_{2} i, h}^{\prime}$

\section{Oxidation of DOC and reduction of $\mathrm{NO}_{3}^{-}, \mathrm{NO}_{2}^{-}$and $\mathrm{N}_{2} \mathrm{O}$ by denitrifiers}

$R_{\mathrm{NO}_{3} i, d}^{\prime}=\boldsymbol{E}_{\mathrm{No}_{\mathbf{x}}} f_{\mathrm{e}}\left(R_{\mathrm{O}_{2} i, d}^{\prime}-R_{\mathrm{O}_{2} i, d}\right)$

$R_{\mathrm{NO}_{3} i, d}=R_{\mathrm{NO}_{3} i, d}^{\prime}\left[\mathrm{NO}_{3}{ }^{-}\right] /\left(\left[\mathrm{NO}_{3}{ }^{-}\right]+K_{\mathrm{NO}_{3}{ }^{d}}\right)$

$R_{\mathrm{NO}_{2} i, d}=\left(R_{\mathrm{NO}_{3} i, d}^{\prime}-R_{\mathrm{NO}_{3} i, d}\right)\left[\mathrm{NO}_{2}\right] /\left(\left[\mathrm{NO}_{2}{ }^{-}\right]+\boldsymbol{K}_{\mathrm{NO}_{2} d}\right)$

$R_{\mathrm{N}_{2} \mathrm{O} i, d}=2\left(R_{\mathrm{NO}_{3} i, d}^{\prime}-R_{\mathrm{NO}_{3} i, d}-R_{\mathrm{NO}_{2} i, d}\right)\left[\mathrm{N}_{2} \mathrm{O}\right] /\left(\left[\mathrm{N}_{2} \mathrm{O}\right]+\boldsymbol{K}_{\mathrm{N}_{2} \mathrm{O} d}\right)$

$X_{\mathrm{DOC} i, d}=X_{\mathrm{DOC} i, d}($ from $[\mathrm{H} 5])+\boldsymbol{F}_{\mathrm{NO}_{\mathbf{x}}}\left(R_{\mathrm{NO}_{3} i, d}+R_{\mathrm{NO}_{2} i, d}\right)+\boldsymbol{F}_{\mathbf{N}_{\mathbf{2}} \mathrm{O}} R_{\mathrm{N}_{2} \mathrm{O} i, d}$

\section{Oxidation of $\mathrm{NH}_{3}$ and reduction of $\mathrm{O}_{2}$ by nitrifiers}

$X_{\mathrm{NH}_{3}, n}^{\prime}=X_{\mathrm{NH}_{3}}^{\prime} M_{\boldsymbol{i}, \boldsymbol{n}, \boldsymbol{a}}\left\{\left[\mathrm{NH}_{3 \mathrm{~S}}\right] /\left(\left[\mathrm{NH}_{3 \mathrm{~S}}\right]+\boldsymbol{K}_{\mathrm{NH}_{3} n}\right)\right\}\left\{\left[\mathrm{CO}_{2 \mathrm{~S}}\right] /\left(\left[\mathrm{CO}_{2 \mathrm{~S}}\right]+\boldsymbol{K}_{\mathbf{C O}_{2}}\right)\right\} f_{\mathrm{t}}$

$R_{\mathrm{O}_{2} i, n}^{\prime}=\mathbf{R} \mathbf{Q}_{\mathrm{NH}_{3}} X_{\mathrm{NH}_{3} i, n}^{\prime}+\mathbf{R} \mathbf{Q}_{\mathbf{C}} X_{\mathrm{C} i, n}^{\prime}$

[H12]

$R_{\mathrm{O}_{2} i, n}=4 \pi n M_{i, n, a} D_{\mathrm{sO}_{2}}\left(r_{\mathrm{m}} r_{\mathrm{w}} /\left(r_{\mathrm{w}}-r_{\mathrm{m}}\right)\right)\left(\left[\mathrm{O}_{2 \mathrm{~S}}\right]-\left[\mathrm{O}_{2 \mathrm{~m} i, n}\right]\right)$

$=R_{\mathrm{O}_{2} i, n}^{\prime}\left[\mathrm{O}_{2 \mathrm{mi}, n}\right] /\left(\left[\mathrm{O}_{2 \mathrm{~m} i, n}\right]+\boldsymbol{K}_{\mathbf{O}_{2^{n}}}\right)$

$X_{\mathrm{NH}_{3} i, n}=X_{\mathrm{NH}_{3} i, n}^{\prime} R_{\mathrm{O}_{2} i, n} / R_{\mathrm{O}_{2} i, n}^{\prime}$ 
Oxidation of $\mathrm{NO}_{2}^{-}$and reduction of $\mathrm{O}_{2}$ by nitrifiers

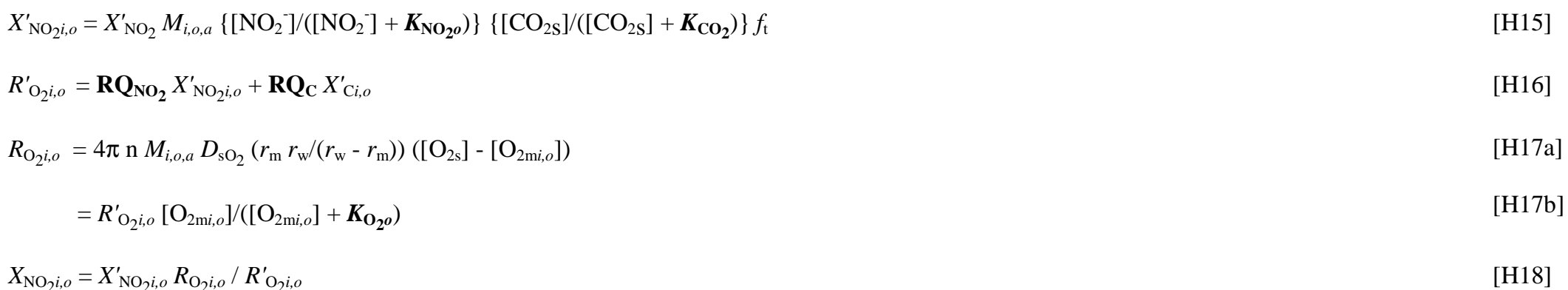

Oxidation of $\mathrm{NH}_{3}$ and reduction of $\mathrm{NO}_{2}^{-}$by nitrifiers

$R_{\mathrm{NO}_{2}, i n}^{\prime}=E_{\mathrm{NO}_{\mathbf{x}}} f_{\mathrm{e}}\left(R_{\mathrm{O}_{2}, n}^{\prime}-R_{\mathrm{O}_{2}, n}\right)$

$R_{\mathrm{NO}_{2} i, n}=R_{\mathrm{NO}_{2} i, n}^{\prime}\left\{\left[\mathrm{NO}_{2}^{-}\right] /\left(\left[\mathrm{NO}_{2}^{-}\right]+\boldsymbol{K}_{\mathrm{NO}_{2}{ }^{n}}\right)\right\}\left\{\left[\mathrm{CO}_{2 \mathrm{~S}}\right] /\left(\left[\mathrm{CO}_{2 \mathrm{~S}}\right]+\boldsymbol{K}_{\mathrm{CO}_{2}}\right)\right\}$

$X_{\mathrm{NH}_{3}, n}=X_{\mathrm{NH}_{3}, n}\left(\right.$ from [H14]) $+0.33 R_{\mathrm{NO}_{2}, i, n}$

Definition of variables in appendix $\mathbf{H}$

\begin{tabular}{lllll}
\hline Name & Definition & Units & Equations & Input Values \\
\hline
\end{tabular}

Subscripts

active component of $\mathbf{M}_{i, m}$

d heterotrophic denitrifier population (subset of $h$ ) 
$\left[\mathrm{CO}_{2 \mathrm{~S}}\right]$

$\left[\mathrm{DOC}_{i}\right]$

$\mathrm{D}_{\mathrm{sO}_{2}}$

$E_{\mathrm{NO}_{\mathbf{x}}}$

$\boldsymbol{F}_{\mathrm{NO}_{\mathbf{x}}}$ heterotrophic community (subset of $m$ )

substrate-microbe complex

kinetic components of $\mathbf{M}_{i, m}$

all microbial communities

autotrophic ammonia oxidizer population (subset of $m$ )

autotrophic nitrite oxidizer population (subset of $m$ )

\section{Variables}

maximum ratio of $M_{i, m, j, \mathrm{~N}}$ to $M_{i, m, j, \mathrm{C}}$ maintained $\mathrm{g} \mathrm{N} \mathrm{g} \mathrm{C}^{-1}$

0.22 and 0.13 for $j=$ by $M_{i, m, j}$

$\mathrm{g} \mathrm{C} \mathrm{m}^{-3}$

$\mathrm{CO}_{2}$ concentration in soil solution

$\mathrm{g} \mathrm{C} \mathrm{m}^{-3}$

concentration of dissolved decomposition

products

aqueous dispersivity-diffusivity of $\mathrm{O}_{2}$

$\mathrm{m}^{2} \mathrm{~h}^{-1}$

$\mathrm{g} \mathrm{N} \mathrm{g} \mathrm{O}_{2}^{-1}$

$\mathrm{e}^{-}$accepted by $\mathrm{NO}_{\mathrm{x}}$ vs. $\mathrm{O}_{2}$ when oxidizing

DOC

$\mathrm{e}^{-}$donated by $\mathrm{C}$ vs. $\mathrm{e}^{-}$accepted by $\mathrm{NO}_{\mathrm{x}}$ when

$\mathrm{g} \mathrm{C} \mathrm{g} \mathrm{N}^{-1}$

oxidizing DOC
$[\mathrm{H} 11, \mathrm{H} 15, \mathrm{H} 20]$

labile and resistant

[H2]

$[\mathrm{H} 4, \mathrm{H} 13, \mathrm{H} 17]$

[H6,H19]

$28 / 32=0.875$

[H10]

$2 / 28=0.43$ 


\begin{tabular}{|c|c|c|c|c|c|}
\hline$F_{\mathrm{N}_{2} \mathrm{O}}$ & $\begin{array}{l}\mathrm{e}^{-} \text {donated by } \mathrm{C} \text { vs. } \mathrm{e}^{-} \text {accepted by } \mathrm{N}_{2} \mathrm{O} \text { when } \\
\text { oxidizing DOC }\end{array}$ & $\mathrm{g} \mathrm{C} \mathrm{g} \mathrm{N}^{-1}$ & {$[\mathrm{H} 10]$} & $6 / 28=0.215$ & \\
\hline$f_{\mathrm{e}}$ & $\begin{array}{l}\text { fraction of electrons not accepted by } \mathrm{O}_{2} \\
\text { transferred to } \mathrm{N} \text { oxides }\end{array}$ & - & {$[\mathrm{H} 6, \mathrm{H} 19]$} & 0.25 & $\begin{array}{l}\text { Koike and } \\
\text { Hattori (1975) }\end{array}$ \\
\hline$f_{\mathrm{t}}$ & temperature function for microbial processes & - & {$[\mathrm{H} 2, \mathrm{H} 11]$} & & \\
\hline$I_{\mathrm{NH}_{4}, i, n j}$ & $\begin{array}{l}\text { mineralization }\left(I_{\mathrm{NH}_{4} i, n, j}<0\right) \text { or immobilization } \\
\left(I_{\mathrm{NH}_{4} i, n, j}>0\right) \text { of } \mathrm{NH}_{4}^{+} \text {by } M_{i, n, j, \mathrm{C}}\end{array}$ & $\mathrm{g} \mathrm{N} \mathrm{m}^{-2} \mathrm{~h}^{-1}$ & {$[\mathrm{H} 1]$} & & \\
\hline$I_{\mathrm{NO}_{3} i, n, j}$ & immobilization $\left(I_{\mathrm{NO}_{3}, i, j, j}>0\right)$ of $\mathrm{NO}_{3}{ }^{-}$by $M_{i, n, j, \mathrm{C}}$ & $\mathrm{g} \mathrm{N} \mathrm{m}^{-2} \mathrm{~h}^{-1}$ & {$[\mathrm{H} 1]$} & & \\
\hline$K_{\mathrm{CO}_{2}}$ & $\begin{array}{l}\text { Michaelis-Menten constant for reduction of } \\
\mathrm{CO}_{2 \mathrm{~S}} \text { by } \mathrm{M}_{i, n, a} \text { and } \mathrm{M}_{i, o, a}\end{array}$ & $\mathrm{~g} \mathrm{C} \mathrm{m}^{-3}$ & {$[\mathrm{H} 11, \mathrm{H} 15, \mathrm{H} 20]$} & 0.15 & \\
\hline$K_{\mathrm{NH}_{3} n}$ & $\begin{array}{l}\text { M-M constant for oxidation of } \mathrm{NH}_{3 \mathrm{~S}} \text { by } \\
\text { nitrifiers }\end{array}$ & $\mathrm{g} \mathrm{N} \mathrm{m}^{-3}$ & [H11] & 0.01 & $\begin{array}{l}\text { Suzuki et al. } \\
\text { (1974) }\end{array}$ \\
\hline$K_{\mathrm{NH}_{4} m}$ & M-M constant for microbial $\mathrm{NH}_{4}{ }^{+}$uptake & $\mathrm{g} \mathrm{N} \mathrm{m}^{-3}$ & [H1] & 0.35 & \\
\hline$K_{\mathrm{NO}_{2} d}$ & $\begin{array}{l}\mathrm{M}-\mathrm{M} \text { constant for reduction of } \mathrm{NO}_{2} \text { by } \\
\text { denitrifiers }\end{array}$ & $\mathrm{g} \mathrm{N} \mathrm{m}^{-3}$ & {$[\mathrm{H} 8]$} & 3.5 & $\begin{array}{l}\text { Yoshinari et al. } \\
\text { (1977) }\end{array}$ \\
\hline $\mathrm{K}_{\mathrm{NO}_{2^{n}}}$ & $\begin{array}{l}\text { M-M constant for reduction of } \mathrm{NO}_{2}^{-} \text {by } \\
\text { nitrifiers }\end{array}$ & $\mathrm{g} \mathrm{N} \mathrm{m}^{-3}$ & {$[\mathrm{H} 20]$} & 3.5 & \\
\hline
\end{tabular}




\begin{tabular}{|c|c|c|c|c|c|}
\hline$K_{\mathrm{NO}_{2}{ }^{o}}$ & $\begin{array}{l}\mathrm{M}-\mathrm{M} \text { constant for oxidation of } \mathrm{NO}_{2}^{-} \text {by } \\
\text { nitrifiers }\end{array}$ & $\mathrm{g} \mathrm{N} \mathrm{m}^{-3}$ & [H15] & 10 & \\
\hline$K_{\mathrm{NO}_{3} d}$ & $\begin{array}{l}\text { M-M constant for reduction of } \mathrm{NO}_{3} \text { by } \\
\text { denitrifiers }\end{array}$ & $\mathrm{g} \mathrm{N} \mathrm{m}^{-3}$ & [H7] & 3.5 & $\begin{array}{l}\text { Yoshinari et al. } \\
\text { (1977) }\end{array}$ \\
\hline$K_{\mathrm{N}_{2} \mathrm{O} d}$ & $\begin{array}{l}\mathrm{M}-\mathrm{M} \text { constant for reduction of } \mathrm{N}_{2} \mathrm{O} \text { by } \\
\text { denitrifiers }\end{array}$ & $\mathrm{g} \mathrm{N} \mathrm{m}^{-3}$ & [H9] & 0.35 & $\begin{array}{l}\text { Yoshinari et al. } \\
\text { (1977) }\end{array}$ \\
\hline $\boldsymbol{K}_{\mathbf{O}^{h}}$ & $\begin{array}{l}\mathrm{M}-\mathrm{M} \text { constant for reduction of } \mathrm{O}_{2 \mathrm{~s}} \text { by } \\
\text { heterotrophs }\end{array}$ & $\mathrm{g} \mathrm{O}_{2} \mathrm{~m}^{-3}$ & {$[\mathrm{H} 4 \mathrm{~b}]$} & 0.064 & Griffin (1972) \\
\hline$K_{\mathbf{O}^{n}}$ & $\begin{array}{l}\text { M-M constant for reduction of } \mathrm{O}_{2 \mathrm{~s}} \text { by } \mathrm{NH}_{3} \\
\text { oxidizers }\end{array}$ & $\mathrm{g} \mathrm{O}_{2} \mathrm{~m}^{-3}$ & [H13b] & 0.32 & $\begin{array}{l}\text { Focht and } \\
\text { Verstraete } \\
\text { (1977) }\end{array}$ \\
\hline$K_{\mathbf{O}_{2} o}$ & $\begin{array}{l}\text { M-M constant for reduction of } \mathrm{O}_{2 \mathrm{~s}} \text { by } \mathrm{NO}_{2}{ }^{-} \\
\text {oxidizers }\end{array}$ & $\mathrm{g} \mathrm{O}_{2} \mathrm{~m}^{-3}$ & {$[\mathrm{H} 17 \mathrm{~b}]$} & 0.32 & $\begin{array}{l}\text { Focht and } \\
\text { Verstraete } \\
\text { (1977) }\end{array}$ \\
\hline$K_{\mathbf{X} h}$ & $\begin{array}{l}\text { M-M constant for oxidation of DOC by } \\
\text { heterotrophs }\end{array}$ & $\mathrm{g} \mathrm{C} \mathrm{m}^{-3}$ & {$[\mathrm{H} 2]$} & 12 & $\begin{array}{l}\text { McGill et al. } \\
\text { (1981) }\end{array}$ \\
\hline$M_{i, h, a}$ & active biomass of heterotrophs & $\mathrm{g} \mathrm{C} \mathrm{m}^{-2}$ & {$[\mathrm{H} 2, \mathrm{H} 7]$} & & \\
\hline$M_{i, n, a}$ & active biomass of $\mathrm{NH}_{3}$ oxidizers & $\mathrm{g} \mathrm{C} \mathrm{m}^{-2}$ & {$[\mathrm{H} 11, \mathrm{H} 13]$} & & \\
\hline
\end{tabular}




\begin{tabular}{|c|c|c|c|}
\hline$M_{i, m, j, \mathrm{C}}$ & C biomass of microbial population $M_{i, m, j}$ & $\mathrm{~g} \mathrm{C} \mathrm{m}^{-2}$ & {$[\mathrm{H} 1]$} \\
\hline$M_{i, m, j, \mathrm{~N}}$ & $\mathrm{~N}$ biomass of microbial population $M_{i, m, j}$ & $\mathrm{~g} \mathrm{~N} \mathrm{~m}^{-2}$ & {$[\mathrm{H} 1]$} \\
\hline$M_{i, o, a}$ & active biomass of $\mathrm{NO}_{2}^{-}$oxidizers & $\mathrm{g} \mathrm{C} \mathrm{m}^{-2}$ & {$[\mathrm{H} 15, \mathrm{H} 17]$} \\
\hline$\left[\mathrm{NH}_{3 \mathrm{~s}}\right]$ & concentration of $\mathrm{NH}_{3}$ in soil solution & $\mathrm{g} \mathrm{N} \mathrm{m}^{-3}$ & [H11] \\
\hline$\left[\mathrm{NH}_{4}^{+}\right]$ & concentration of $\mathrm{NH}_{4}{ }^{+}$in soil solution & $\mathrm{g} \mathrm{N} \mathrm{m}^{-3}$ & {$[\mathrm{H} 1]$} \\
\hline$\left[\mathrm{NO}_{2}\right]$ & concentration of $\mathrm{NO}_{2}$ in soil solution & $\mathrm{g} \mathrm{N} \mathrm{m}^{-3}$ & {$[\mathrm{H} 8, \mathrm{H} 15, \mathrm{H} 20]$} \\
\hline$\left[\mathrm{NO}_{3}\right]$ & concentration of $\mathrm{NO}_{3}$ in soil solution & $\mathrm{g} \mathrm{N} \mathrm{m}^{-3}$ & {$[\mathrm{H} 7]$} \\
\hline$\left[\mathrm{N}_{2} \mathrm{O}\right]$ & concentration of $\mathrm{N}_{2} \mathrm{O}$ in soil solution & $\mathrm{g} \mathrm{N} \mathrm{m}^{-3}$ & [H9] \\
\hline$n$ & number of microbes & $g^{-1}$ & {$[\mathrm{H} 13, \mathrm{H} 17]$} \\
\hline$\left[\mathrm{O}_{2 \mathrm{~m}, h}\right]$ & $\mathrm{O}_{2}$ concentration at heterotrophic surfaces & $\mathrm{g} \mathrm{O}_{2} \mathrm{~m}^{-3}$ & {$[\mathrm{H} 7]$} \\
\hline$\left[\mathrm{O}_{2 \mathrm{~m}, n}\right]$ & $\mathrm{O}_{2}$ concentration at $\mathrm{NH}_{3}$ oxidizer surfaces & $\mathrm{g} \mathrm{O}_{2} \mathrm{~m}^{-3}$ & [H13] \\
\hline$\left[\mathrm{O}_{2 \mathrm{~m} i, o}\right]$ & $\mathrm{O}_{2}$ concentration at $\mathrm{NO}_{2}^{-}$oxidizer surfaces & $\mathrm{g} \mathrm{O}_{2} \mathrm{~m}^{-3}$ & [H17] \\
\hline$\left[\mathrm{O}_{2 \mathrm{~s}}\right]$ & $\mathrm{O}_{2}$ concentration in soil solution & $\mathrm{g} \mathrm{O}_{2} \mathrm{~m}^{-3}$ & {$[\mathrm{H} 7, \mathrm{H} 13, \mathrm{H} 17]$} \\
\hline$R_{\mathrm{NO}_{2}, d}$ & $\mathrm{NO}_{2}$ reduction by denitrifiers & $\mathrm{g} \mathrm{N} \mathrm{m}^{-2} \mathrm{~h}^{-1}$ & {$[\mathrm{H} 8, \mathrm{H} 9, \mathrm{H} 10]$} \\
\hline$R_{\mathrm{NO}_{2}, i n}^{\prime}$ & rate of $\mathrm{NO}_{2}{ }^{-}$reduction by $\mathrm{NH}_{3}$ oxidizers under & $\mathrm{g} \mathrm{N} \mathrm{m}^{-2} \mathrm{~h}^{-1}$ & {$[\mathrm{H} 19, \mathrm{H} 20]$} \\
\hline
\end{tabular}

non-limiting $\left[\mathrm{NO}_{2}{ }^{-}\right]$and $\left[\mathrm{CO}_{2 \mathrm{~S}}\right]$ 


\begin{tabular}{|c|c|c|c|}
\hline$R_{\mathrm{NO}_{2}, i n}$ & $\begin{array}{l}\text { rate of } \mathrm{NO}_{2}^{-} \text {reduction by } \mathrm{NH}_{3} \text { oxidizers under } \\
\text { ambient }\left[\mathrm{NO}_{2}^{-}\right] \text {and }\left[\mathrm{CO}_{2 \mathrm{~S}}\right]\end{array}$ & $\mathrm{g} \mathrm{N} \mathrm{m}^{-2} \mathrm{~h}^{-1}$ & {$[\mathrm{H} 20, \mathrm{H} 21]$} \\
\hline$R_{\mathrm{NO}_{3} i, d}^{\prime}$ & $\begin{array}{l}\mathrm{NO}_{3}^{-} \text {reduction by denitrifiers under non- } \\
\text { limiting }\left[\mathrm{NO}_{3}{ }^{-}\right]\end{array}$ & $\mathrm{g} \mathrm{N} \mathrm{m}^{-2} \mathrm{~h}^{-1}$ & {$[\mathrm{H} 6, \mathrm{H} 7, \mathrm{H} 8, \mathrm{H} 9]$} \\
\hline$R_{\mathrm{NO}_{3}, d}$ & $\begin{array}{l}\mathrm{NO}_{3}^{-} \text {reduction by denitrifiers under ambient } \\
{\left[\mathrm{NO}_{3}^{-}\right]}\end{array}$ & $\mathrm{gN} \mathrm{m}^{-2} \mathrm{~h}^{-1}$ & {$[\mathrm{H} 7, \mathrm{H} 8, \mathrm{H} 9, \mathrm{H} 10]$} \\
\hline$R_{\mathrm{N}_{2} \mathrm{O} i, d}$ & $\mathrm{~N}_{2} \mathrm{O}$ reduction by denitrifiers & $\mathrm{g} \mathrm{N} \mathrm{m}^{-2} \mathrm{~h}^{-1}$ & {$[\mathrm{H} 9, \mathrm{H} 10]$} \\
\hline$R_{\mathrm{O}_{2} i, d}^{\prime}$ & $\begin{array}{l}\text { rate of } \mathrm{O}_{2 \mathrm{~S}} \text { reduction by denitrifiers under non- } \\
\text { limiting }\left[\mathrm{O}_{2 \mathrm{~S}}\right]\end{array}$ & $\mathrm{g} \mathrm{O}_{2} \mathrm{~m}^{-2} \mathrm{~h}^{-1}$ & {$[\mathrm{H} 6]$} \\
\hline$R_{\mathrm{O}_{2} i, d}$ & $\begin{array}{l}\text { rate of } \mathrm{O}_{2 \mathrm{~S}} \text { reduction by denitrifiers under } \\
\text { ambient }\left[\mathrm{O}_{2 \mathrm{~S}}\right]\end{array}$ & $\mathrm{g} \mathrm{O}_{2} \mathrm{~m}^{-2} \mathrm{~h}^{-1}$ & {$[\mathrm{H} 6]$} \\
\hline$R_{\mathrm{O}_{2} i, h}$ & $\begin{array}{l}\text { rate of } \mathrm{O}_{2 \mathrm{~S}} \text { reduction by heterotrophs under } \\
\text { non-limiting }\left[\mathrm{O}_{2 \mathrm{~S}}\right]\end{array}$ & $\mathrm{g} \mathrm{O}_{2} \mathrm{~m}^{-2} h^{-1}$ & {$[\mathrm{H} 3, \mathrm{H} 4, \mathrm{H} 5]$} \\
\hline$R_{\mathrm{O}_{2}, i, h}$ & $\begin{array}{l}\text { rate of } \mathrm{O}_{2 \mathrm{~S}} \text { reduction by heterotrophs under } \\
\text { ambient }\left[\mathrm{O}_{2 \mathrm{~S}}\right]\end{array}$ & $\mathrm{g} \mathrm{O}_{2} \mathrm{~m}^{-2} h^{-1}$ & {$[\mathrm{H} 4, \mathrm{H} 5]$} \\
\hline$R_{\mathrm{O}_{2}, n}^{\prime}$ & $\begin{array}{l}\text { rate of } \mathrm{O}_{2 \mathrm{~S}} \text { reduction by } \mathrm{NH}_{3} \text { oxidizers under } \\
\text { non-limiting }\left[\mathrm{O}_{2 \mathrm{~S}}\right]\end{array}$ & $\mathrm{g} \mathrm{O}_{2} \mathrm{~m}^{-2} \mathrm{~h}^{-1}$ & {$[\mathrm{H} 12, \mathrm{H} 13 . \mathrm{H} 14, \mathrm{H} 19$} \\
\hline
\end{tabular}




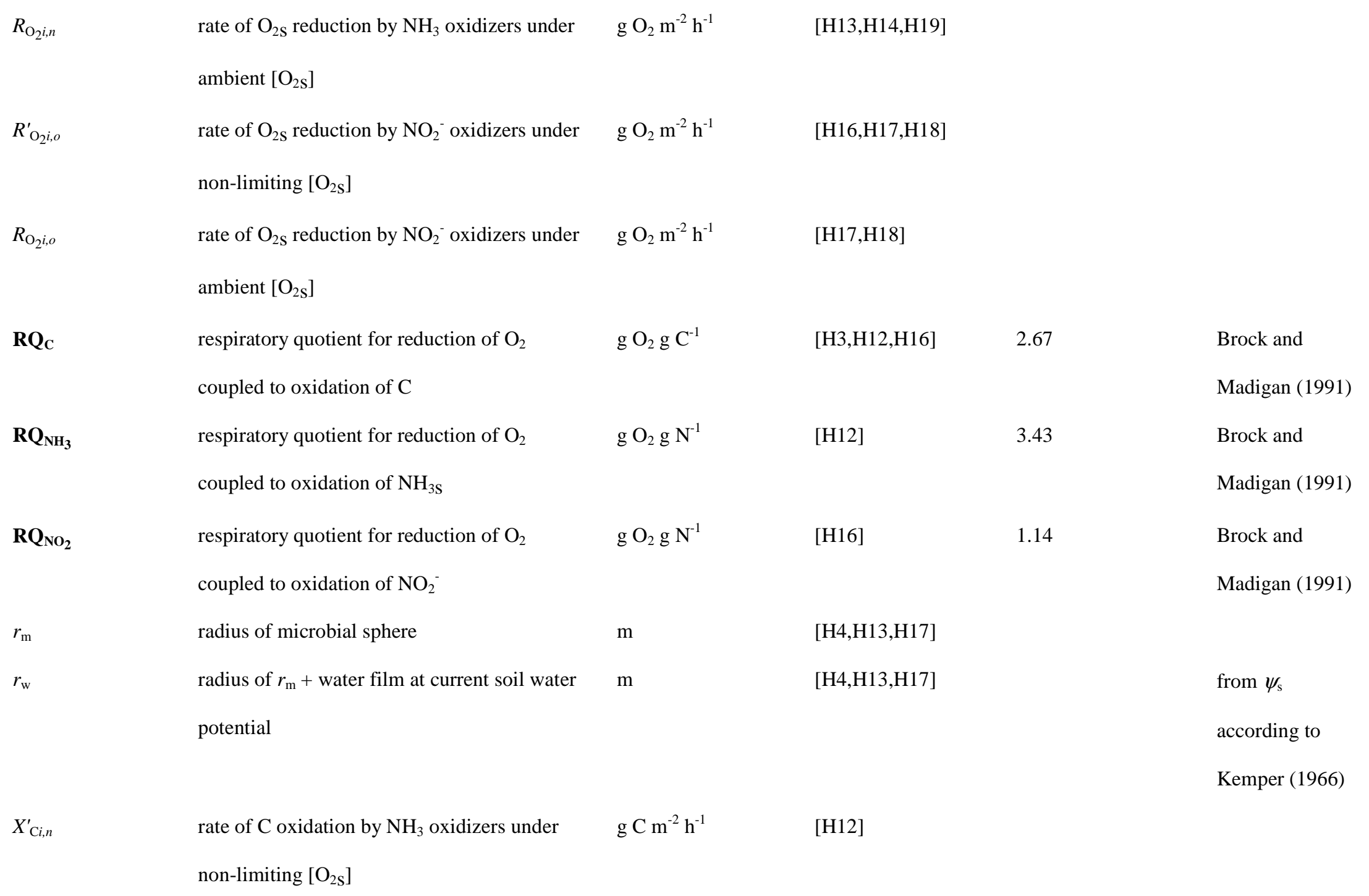




\begin{tabular}{|c|c|c|c|c|c|}
\hline$X_{\mathrm{C}, o}^{\prime}$ & $\begin{array}{l}\text { rate of } \mathrm{C} \text { oxidation by } \mathrm{NO}_{2}^{-} \text {oxidizers under } \\
\text { non-limiting }\left[\mathrm{O}_{2 \mathrm{~s}}\right]\end{array}$ & $\mathrm{g} \mathrm{C} \mathrm{m}^{-2} \mathrm{~h}^{-1}$ & [H16] & & \\
\hline$X_{\mathrm{DOC}}^{\prime}$ & $\begin{array}{l}\text { specific rate of DOC oxidation by heterotrophs } \\
\text { at } 25^{\circ} \mathrm{C} \text { under non-limiting }[\mathrm{DOC}] \text { and }\left[\mathrm{O}_{2 \mathrm{~s}}\right]\end{array}$ & $\mathrm{gC} \mathrm{g} \mathrm{C}^{-1} \mathrm{~h}^{-1}$ & {$[\mathrm{H} 2]$} & 0.125 & $\begin{array}{l}\text { Shields et al. } \\
\text { (1973) }\end{array}$ \\
\hline$X_{\text {DOC } i, h}^{\prime}$ & $\begin{array}{l}\text { rate of DOC oxidation by heterotrophs under } \\
\text { non-limiting }\left[\mathrm{O}_{2 \mathrm{~S}}\right]\end{array}$ & $\mathrm{g} \mathrm{N} \mathrm{m}^{-2} \mathrm{~h}^{-1}$ & {$[\mathrm{H} 2, \mathrm{H} 3, \mathrm{H} 5]$} & & \\
\hline$X_{\mathrm{DOC} i, h}$ & $\begin{array}{l}\text { rate of DOC oxidation by heterotrophs under } \\
\text { ambient }\left[\mathrm{O}_{2 \mathrm{~S}}\right]\end{array}$ & $\mathrm{g} \mathrm{N} \mathrm{m}^{-2} \mathrm{~h}^{-1}$ & [H5] & & \\
\hline$X_{\mathrm{DOC}, d}$ & $\begin{array}{l}\text { rate of DOC oxidation by heterotrophs under } \\
\text { ambient }\left[\mathrm{O}_{2 \mathrm{~S}}\right] \text { and }\left[\mathrm{NO}_{\mathrm{x}}\right]\end{array}$ & $\mathrm{g} \mathrm{N} \mathrm{m}^{-2} \mathrm{~h}^{-1}$ & [H10] & & \\
\hline$X_{\mathrm{NH}_{3}}^{\prime}$ & $\begin{array}{l}\text { specific rate of } \mathrm{NH}_{3} \text { oxidation by } \mathrm{NH}_{3} \\
\text { oxidizers at } 25^{\circ} \mathrm{C} \text { under non-limiting }\left[\mathrm{O}_{2 \mathrm{~S}}\right]\end{array}$ & $\mathrm{g} \mathrm{N} \mathrm{g} \mathrm{C}^{-1} \mathrm{~h}^{-1}$ & [H11]] & 0.625 & $\begin{array}{l}\text { Belser and } \\
\text { Schmidt (1980) }\end{array}$ \\
\hline$X_{\mathrm{NH}_{3}, n}$ & $\begin{array}{l}\text { rate of } \mathrm{NH}_{3} \text { oxidation by } \mathrm{NH}_{3} \text { oxidizers } \\
\text { coupled with reduction of } \mathrm{O}_{2}+\mathrm{NO}_{2}^{-} \text {under } \\
\text { ambient }\left[\mathrm{O}_{2 \mathrm{~S}}\right]\end{array}$ & $\mathrm{g} \mathrm{N} \mathrm{m}^{-2} \mathrm{~h}^{-1}$ & {$[\mathrm{H} 14, \mathrm{H} 21]$} & & \\
\hline$X_{\mathrm{NH}_{3}, i, n}^{\prime}$ & $\begin{array}{l}\text { rate of } \mathrm{NH}_{3} \text { oxidation by } \mathrm{NH}_{3} \text { oxidizers under } \\
\text { non-limiting }\left[\mathrm{O}_{2 \mathrm{~S}}\right]\end{array}$ & $\mathrm{g} \mathrm{N} \mathrm{m}^{-2} \mathrm{~h}^{-1}$ & [H11,H12,H14] & & \\
\hline
\end{tabular}


$X_{\mathrm{NO}_{2} i, o}^{\prime}$

$X_{\mathrm{NO}_{2} i, o}$

$X_{\mathrm{NO}_{2}}^{\prime}$ rate of $\mathrm{NO}_{2}^{-}$oxidation by $\mathrm{NO}_{2}{ }^{-}$oxidizers under $\mathrm{g} \mathrm{N} \mathrm{m}^{-2} \mathrm{~h}^{-1}$

non-limiting $\left[\mathrm{O}_{2 \mathrm{~s}}\right]$

rate of $\mathrm{NO}_{2}^{-}$oxidation by $\mathrm{NO}_{2}^{-}$oxidizers

$\mathrm{g} \mathrm{N} \mathrm{m}{ }^{-2} h^{-1}$

coupled with reduction of $\mathrm{O}_{2}$ under ambient

$\left[\mathrm{O}_{2 \mathrm{~S}}\right]$

specific rate of $\mathrm{NO}_{2}^{-}$oxidation by $\mathrm{NO}_{2}$

$\operatorname{g~N~g~C~}{ }^{-1} h^{-1}$

[H15]

2.5

Belser (1977)

oxidizers at $25{ }^{\circ} \mathrm{C}$ under non-limiting $\left[\mathrm{O}_{2 \mathrm{~S}}\right]$ 


\section{References}

Addiscott, T. M.: Kinetics and temperature relationships of mineralization and nitrification in Rothamsted soils with differing histories, J. Soil Sci., 34, 343-353, doi: 10.1111/j.1365-2389.1983.tb01040.x, 1983.

559 Anthony, C.: The Biochemistry of Methylotrophs, Academic Press, London, UK, 1982.

560 Barber, S. A., and Silberbush, M.: Plant root morphology and nutrient uptake, in: Roots, Nutrient 561 and Water Influx, and Plant Growth, edited by: Barber, S. A., and Bouldin, D. R., Amer. Soc. Agron. Spec. Publ. no. 49, Madison, WI, 65-87, 1984.

563 Barnes, B.V., Zak, D. R., Denton, S. R., and Spurr, S. H.: Forest Ecology, $4^{\text {th }}$ edition, $564 \quad$ Wiley and Sons, NY, 1998.

565 Belser, L.W., and Schmidt, E. L.: Growth and oxidation kinetics of the three genera of 566 ammonia oxidizers, FEMS Microbiol. Lett., 7, 213-216, doi: 10.1111/j.1574$567 \quad$ 6941.1980.tb01628.x, 1980.

568 Belser, L.W.: Nitrate reduction to nitrite, a possible source of nitrite for growth of nitrite569 oxidizing bacteria, Appl. Environ. Micro., 34, 403-410, 1977.

570 Bernacchi, C. J., Singsaas, E. L., Pimentel, C., Portis, A. L., and Long, S. P.: Improved 571 temperature response functions for models of rubisco-limited photosynthesis, Plant 572 Cell Environ., 24, 253-259, doi: 10.1111/j.1365-3040.2001.00668.x, 2001.

573 Bernacchi, C. J., Pimentel, C., and Long, S. P.: In vivo temperature response functions of 574 parameters required to model RuBP-limited photosynthesis, Plant Cell Environ., 26, 575 1419-1430, doi: 10.1046/j.0016-8025.2003.01050.x, 2003. 
Berry, J. A., and Farquhar, G. D.: The $\mathrm{CO}_{2}$ concentrating function of photosynthesis: a biochemical model, in: Proceedings of the 4th Interntl. Congress on Photosynthesis, edited by: Hall, D., Coombs, J., and Goodwin, T., Biochemical Society, London, 119$131,1978$.

Brock, T. D., and Madigan, M. T.: Biology of Microorganisms, $6^{\text {th }}$ edition, Prentice Hall, NJ, 1991.

Campbell, G. S.: Soil Physics with BASIC, Elsevier, Netherlands, 1985.

Conrad, R.: Capacity of aerobic microorganisms to utilize and grow on atmospheric trace gases $\left(\mathrm{H}_{2}, \mathrm{CO}, \mathrm{CH}_{4}\right)$, in: Current Perspectives in Microbial Ecology, edited by: Klug, M. J., and Reddy, C. A., Amer. Soc. Microbiol. Wash. DC, 461-467, 1984.

de Vries, D.A.: Thermal properties of soils, in: Physics of Plant Environment, edited by: van Wijk, R., North Holland Publishing Co., Amsterdam, Netherlands, 210-235, 1963.

Dimitrov, D. D., Grant, R. F., Lafleur, P. M., and Humphreys, E. R.: Modelling subsurface hydrology of Mer Bleue bog, Soil Sci. Soc. Am. J., 74, 680-694, doi:10.2136/sssaj2009.0148, 2010.

Doussan C., Vercambre, G., and Pagès, L.: Modelling of the hydraulic architecture of root systems: An integrated approach to water absorption-distribution of axial and radial conductances in maize, Ann. Bot., 81, 225-232, doi: 10.1006/anbo.1997.0541, 1998.

Edwards, G., and Walker, D.: $\mathrm{C}_{3}, \mathrm{C}_{4}$ : Mechanisms, and Cellular and Environmental Regulation of Photosynthesis, Univ. of California Press, Berkely, CA, 1983. 
597 Farquhar, G. D., von Caemmerer, S., and Berry, J. A.: A biochemical model of photosynthetic $598 \mathrm{CO}_{2}$ assimilation in leaves of $\mathrm{C}_{3}$ species, Planta, 149, 78-90, doi: 10.1007/BF00386231, $599 \quad 1980$.

600 Focht, D. D., and Verstraete, W.: Biochemical ecology of nitrification and denitrification, Adv. $601 \quad$ Micro. Ecol., 1, 135-214, 1977.

602 Furbank, F. T., and Hatch, M. D.: Mechanism of $\mathrm{C}_{4}$ photosynthesis, The size and composition 603 of the inorganic carbon pool in bundle sheath cells, Plant Physiol., 85, 958-964, doi: $604 \quad 10.1104 / p p .85 .4 .958,1987$.

605 Grant, R. F.: Mathematical modelling of nitrous oxide evolution during nitrification, Soil Biol. 606 Biochem., 27, 1117-1125, doi: 10.1016/0038-0717(95)00038-G, 1995.

607 Grant, R. F.: Mathematical modelling of nitrous oxide evolution during nitrification, Soil Biol. 608 Biochem., 27, 1117-1125, doi: 10.1016/0038-0717(95)00038-G, 1995.

609 Grant, R. F.: Simulation in ecosys of root growth response to contrasting soil water and 610 nitrogen, Ecol. Model., 107, 237-264, doi: 10.1016/S0304-3800(97)00221-4, 1998a.

611 Grant, R. F.: Simulation of methanogenesis in the mathematical model ecosys, Soil Biol. 612 Biochem., 30, 883-896, doi: 10.1016/S0038-0717(97)00218-6, 1998b.

613 Grant, R. F.: Simulation of methanotrophy in the mathematical model ecosys, Soil Biol. 614 Biochem., 31, 287-297, doi: 10.1016/S0038-0717(98)00119-9, 1999.

615 Grant, R. F.: A review of the Canadian ecosystem model ecosys, in: Modeling Carbon 616 and Nitrogen Dynamics for Soil Management, edited by: Shaffer, M., CRC Press, 617 Boca Raton, Fla., 173-264, 2001.

618 Grant, R. F.: Modelling topographic effects on net ecosystem productivity of boreal black 619 spruce forests, Tree Physiol., 24, 1-18, doi: 10.1093/treephys/24.1.1, 2004. 
620 Grant, R. F., and Flanagan, L. B.: Modeling stomatal and nonstomatal effects of water 621 deficits on $\mathrm{CO}_{2}$ fixation in a semiarid grassland, J. Geophys. Res.-Biogeosci., 112, 622 G03011, doi: 10.1029/2006JG000302, 2007.

623 Grant, R. F., and Heaney, D. J.: Inorganic phosphorus transformation and transport in 624 soils: mathematical modelling in ecosys, Soil Sci. Soc. Am. J., 61, 752-764, doi: $625 \quad 10.2136 /$ sssaj1997.03615995006100030008x, 1997.

626 Grant, R. F., and Hesketh, J. D.: Canopy structure of maize (Zea mays L.) at different 627 populations: simulation and experimental verification, Biotronics, 21, 11-24, 1992.

628 Grant, R. F., and Rochette, P.: Soil microbial respiration at different temperatures and 629 water potentials: Theory and mathematical modeling, Soil Sci. Soc. Am. J., 58, 1681630 1690, doi: 10.2136/sssaj1994.03615995005800060015x, 1994.

631 Grant, R. F., Juma, N. G., and McGill, W. B.: Simulation of carbon and nitrogen 632 transformations in soils: Mineralization, Soil Biol. Biochem., 25, 1317-1329, doi: $633 \quad 10.1016 / 0038-0717(93) 90046-E, 1993 a$.

634 Grant, R. F., Juma, N. G., and McGill, W. B.: Simulation of carbon and nitrogen 635 transformations in soils: Microbial biomass and metabolic products, Soil Biol. 636 Biochem., 25, 1331-1338, doi: 10.1016/0038-0717(93)90047-F, 1993b.

637 Grant, R. F., Wall, G. W., Kimball, B. A., Frumau, K. F. A., Pinter Jr., P. J., Hunsaker, D. 638 J., and Lamorte, R. L.: Crop water relations under different $\mathrm{CO}_{2}$ and irrigation: testing 639 of ecosys with the Free Air $\mathrm{CO}_{2}$ Enrichment (FACE) experiment, Agricult. Forest $640 \quad$ Meteorol., 95, 27-51, doi: 10.1016/S0168-1923(99)00017-9, 1999. 
641 Grant, R.F., Amrani, M., Heaney, D. J., Wright, R., and Zhang, M.: Mathematical 642 modelling of phosphorus losses from land application of hog and cattle manure, J. 643 Environ. Qual., 33, 210-231, doi: 10.2134/jeq2004.2100, 2004.

644 Grant, R. F., Kimball, B. A., Wall, G. W., Triggs, J. M., Brooks, T. J., Pinter Jr., P. J., 645 Conley, M. M., Ottman, M. J., Lamorte, R. L., Leavitt, S. W., Thompson, T. L., and 646 Matthias, A. D.: Modeling elevated carbon dioxide effects on water relations, water 647 use, and growth of irrigated sorghum, Agron. J., 96, 1693-1705, doi: $648 \quad$ 10.2134/agronj2004.1693, 2004.

649 Grant, R. F., Black, T. A., Humphreys, E. R., and Morgenstern, K.: Changes in net 650 ecosystem productivity with forest age following clearcutting of a coastal Douglas fir 651 forest: testing a mathematical model with eddy covariance measurements along a 652 forest chronosequence, Tree Physiol., 27, 115-131, doi: 0.1093/treephys/27.1.115, 6532007

654 Grant, R. F., Barr, A. G., Black, T. A., Margolis, H. A., McCaughey, J. H., and 655 Trofymow, J. A.: Net ecosystem productivity of temperate and boreal forests after 656 clearcutting-a Fluxnet-Canada synthesis, Tellus B, 62, 475-496, doi: 10.1111/j.1600$657 \quad$ 0889.2010.00500.x, 2010.

658 Green, R. E., and Corey, R. B.: Calculation of hydraulic conductivity: A further 659 evaluation of some predictive methods, Soil Sci. Soc. Am. Proc., 35, 3-8, doi: $660 \quad 10.2136 /$ sssaj1971.03615995003500010010x, 1971.

661 Griffin, D. M.: Ecology of Soil Fungi, Syracuse Univ. Press, Syracuse NY, 1972. 
662 Jiao, J. A., and Chollet, R.: Light/dark regulation of maize leaf phosphoenol pyruvate 663 carboxylase by in vivo phosphorylation, Arch. Biochem. Biophys., 261, 409-417, doi: $664 \quad 10.1016 / 0003-9861(88) 90357-8,1988$.

665 Kemper, W. D., and Rollins, J. B.: Osmotic efficiency coefficients across compacted 666 clays, Soil Sci. Soc. Amer. J., 30, 529-534, doi: 10.2136/sssaj1966.0361599500300 $667 \quad 0050005 x, 1966$.

668 Kimmins, J. P.: Forest Ecology, Pearson Prentice Hall, NJ, 2004.

669 Koike, I., and Hattori, A.: Growth yield of a denitrifying bacterium, Pseudomonas 670 denitrificans, under aerobic and denitrifying conditions, Soc. General Microbiol., 88, $671 \quad$ 1-10, doi: 10.1099/00221287-88-1-1, 1975.

672 Laisk, A., and Edwards, G. E.: A mathematical model of $\mathrm{C}_{4}$ photosynthesis: The 673 mechanism of concentrating $\mathrm{CO}_{2}$ in NADP-malic enzyme type species, Photosyn. $674 \quad$ Res., 66, 199-224, doi: 10.1023/A:1010695402963, 2000.

675 Larcher, W.: Physiological Plant Ecology, $4^{\text {th }}$ edition, Springer-Verlag, Berlin, 2001.

676 Lawlor, D.: Photosynthesis: Molecular, Physiological and Environmental Processes, 677 Longman Group, Essex, UK, 1993.

678 Lawrence, A. L.: Anaerobic biological treatment processes, in: Advances in Chemistry 679 Series 105, edited by: Gould, R. F., Amer. Chem. Soc. Wash. DC, 163-189, 1971.

680 Leegood, R. C.: Transport during $\mathrm{C}_{4}$ photosynthesis, in: Advances in Photosynthesis: 681 Physiology and Metabolism, edited by: Leegood, R. C., Sharkey, T.D. and von 682 Caemmerer, S., Kluwer Academic Publishers, Dordrecht, 449-469, 2000. 
683 Lizama, H. M., and Suzuki, I.: Kinetics of sulfur and pyrite oxidation by Thiobacillus

684 thiooxidans. Competitive inhibition by increasing concentrations of cells, Can. J.

$685 \quad$ Microbio., 37, 182-187, doi: 10.1139/m91-028, 1990.

686 Luxmoore, R. J., Stolzy, L. H., and Letey J.: Oxygen diffusion in the soil-plant system. I. a 687 model, Agron. J., 62, 317-322, doi: 10.2134/agronj1970.00021962006200030003x, 1970a.

688 Luxmoore, R. J., Stolzy, L. H., and Letey, J.: Oxygen diffusion in the soil-plant system. II. 689 Respiration rate, permeability, and porosity of consecutive excised segments of maize and 690 rice roots, Agron. J., 62, 322-324, doi: 10.2134/agronj1970.00021962006200030004x, 1970b.

691 McGill, W. B., Hunt, H. W., Woodmansee, R. G., and Reuss, J. O.: Phoenix, a model of 692 the dynamics of carbon and nitrogen in grassland soils, in: Terrestrial Nitrogen Cycles, 693 edited by: Clark, F. E., and Rosswall, T., Ecological Bulletins 33, 49-115, 1981.

694 Medrano, H., Escalona, J. M., Bota, J., Gulías, J., and Flexas, J.: Regulation of 695 photosynthesis of $C_{3}$ plants in response to progressive drought: stomatal conductance 696 as a reference parameter, Ann. Bot., 89, 895-905, doi: 10.1093/aob/mcf079, 2002.

697 Millington, R. J., and Quirk, J. M.: Transport in porous media, in: $7^{\text {th }}$ Trans. Int. Congr. 698 Soil Sci. vol. 1, edited by: van Beren, F. A., Madison, WI, Elsevier, Amsterdam, 97$699 \quad 106,1960$.

700 Mosey, F. E.: Kinetic descriptions of anaerobic digestion, in: Third International 701 Symposium on Anaerobic Digestion, Univ. Cambridge, Boston, MS, 37-52, 1983.

702 Perrier, A.: Land surface processes: vegetation, in: Atmospheric General Circulation 703 Models, edited by: Eagleson, P. S., Cambridge Univ. Press., Cambridge, UK, 395-448, $704 \quad 1982$. 
Pirt, S. J.: Principles of Microbe and Cell Cultivation, Blackwell Scientific, Oxford, $706 \quad$ UK, 1975.

707 Postgate, J.: Nitrogen Fixation, $3^{\text {rd }}$ edition, Cambridge Univ. Press, Cambridge, UK, $708 \quad 1998$

709 Robinson, J. A., and Tiedje, J. M.: Kinetics of hydrogen consumption by rumen fluid, 710 digestor sludge and sediment, Appl. Environ. Microbiol., 44, 1374-1384, 1982.

711 Sawada, S., Sakamoto, T., Sato, M., Kasai, M., and Usuda, H.: Photosynthesis with 712 single-rooted Amaranthus leaves. II. Regulation of Ribulose-1,5-Bisphosphate 713 Carboxylase, Phosphoenolpyruvate Carboxylase, NAD-Malic Enzyme and NAD714 Malate Dehydrogenase and coordination between PCR and $\mathrm{C}_{4}$ photosynthetic 715 metabolism in response to changes in the source-sink balance, Plant Cell Physiol., 716 43, 1293-301, doi: 10.1093/pcp/pcf153, 2002.

717 Schink, B.: Energetics of syntrophic cooperation in methanogenic degradation, 718 Microbiol. Mol. Biol. Rev., 61, 262-280, 1997.

719 Seeman, J. R., Badger, M. R., and Berry, J. A.: Variations in the specific activity of 720 ribulose-1,5-bisphosphate carboxylase between species utilizing differing 721 photosynthetic pathways, Plant Physiol., 74, 791-794, doi: 10.1104/pp.74.4.791, $722 \quad 1984$

723 Sharpe, P. S. H., and DeMichelle, D. W.: Reaction kinetics of poikilothermic 724 development, J. Theor. Biol., 64, 649-670, doi: 10.1016/0022-5193(77)90265-X, $725 \quad 1977$ 
Shea, T. G., Pretorius, W. E., Cole, R. D., and Pearson, E. A.: Kinetics of hydrogen assimilation in the methane fermentation, Water Res., 2, 833-848, doi: 10.1016/0043-1354(68)90038-9, 1968.

Shields, J. A., Paul, E. A., Lowe, W. E., and Parkinson, D.: Turnover of microbial tissue in soil under field conditions, Soil Biol. Biochem., 5, 753-764, doi: 10.1016/00380717(73)90020-5, 1973.

Shulten, H.-R., and Schnitzer, M.: Chemical model structures for organic matter and soils, Soil Sci., 162, 115-130, 1997.

Skopp, J.: Oxygen uptake and transfer in soils: analysis of the air-water interfacial area, Soil Sci. Soc. Amer. J., 49, 1327-1331, doi: 10.2136/sssaj1985.036159950049000 $60001 x, 1985$.

Smith, M. R., and Mah, R. A.: Growth and methanogenesis by Methanosarcina strain 227 on acetate and methanol, Appl. Environ. Microbiol., 36, 870-879, 1978.

Suzuki, I., Dular, U., and Kwok, S. C.: Ammonia or ammonium ion as substrate for oxidation by Nitrosomonas europeae cells and extracts, J. Bacteriol., 120, 556-558, 1974.

van Bavel, C. H. M., and Hillel, D. I.: Calculating potential and actual evaporation from a bare soil surface by simulation of concurrent flow of water and heat, Agric. Meteorol., 17, 453-476, doi: 10.1016/0002-1571(76)90022-4, 1976.

Veen, B. W.: Relation between root respiration and root activity, Plant Soil, 63, 73-76., doi: 10.1007/BF02374259, 1981.

Waring, R. H., and Running, S. W.: Forest Ecosystems: Analysis at Multiple Scales, $2^{\text {nd }}$ edition, Academic Press, London, UK, 1998. 
749 Wilhelm, E., Battino, R., and Wilcock, R. J.: Low-pressure solubility of gases in liquid $750 \quad$ water, Chem. Rev., 77, 219-262, doi: 10.1021/cr60306a003, 1977.

751 Williams, D. G., Gempko, V., Fravolini, A., Leavitt, S. W., Wall, G. W., Kimball, B. A.,

752 Pinter Jr., P. J., LaMorte, R., and Ottman, M.: Carbon isotope discrimination by 753 Sorghum bicolor under $\mathrm{CO}_{2}$ enrichment and drought, New Phytol., 150, 285-293, doi: $754 \quad$ 10.1046/j.1469-8137.2001.00093.x, 2001.

755 Wofford, N. G., Beaty, P. S., and McInerey, M. J.: Preparation of cell-free extracts and 756 the enzymes involved in fatty acid metabolism in Syntrophomonas wolfei, J. $757 \quad$ Bacteriol., 167, 189-195, 1986.

758 Yoshinari, T., Hynes, R., and Knowles, R.: Acetylene inhibition of nitrous oxide 759 reduction and measurement of denitrification and nitrogen fixation in soil, Soil Biol. $760 \quad$ Biochem., 9, 177-183, doi: 10.1016/0038-0717(77)90072-4, 1977.

761 Zehnder, A. J. B., and Wuhrmann, K.: Physiology of a Methanobacterium strain AZ, $762 \quad$ Arch. Microbiol., 111, 199-205, doi: 10.1007/BF00549357, 1977.

763 Zehnder, A. J. B., Huser, B. A., Brock, T. D. and Wuhrmann, K.: Characterization of an 764 acetate-decarboxylating, non-hydrogen-oxidizing methane bacterium, Arch. $765 \quad$ Microbiol., 124, 1-11, doi: 10.1007/BF00407022, 1980. 